\title{
symmetry
}

Kinetic Theory and

Swarming Tools to

Modeling Complex

Systems-

Symmetry problems

in the Science

of Living Systems

Edited by

Nicola Bellomo

Printed Edition of the Special Issue Published in Symmetry 
Kinetic Theory and Swarming

Tools to Modeling Complex

Systems-Symmetry problems in the Science of Living Systems 



\section{Kinetic Theory and Swarming Tools to Modeling Complex Systems-Symmetry problems in the Science of Living Systems}

Special Issue Editor

Nicola Bellomo 
Special Issue Editor

Nicola Bellomo

University of Granada

Spain

\section{Editorial Office}

MDPI

St. Alban-Anlage 66

4052 Basel, Switzerland

This is a reprint of articles from the Special Issue published online in the open access journal Symmetry (ISSN 2073-8994) (available at: https://www.mdpi.com/journal/symmetry/special_issues/Kinetic_ Theory_Swarming_Tools_Modeling_Complex_Systems).

For citation purposes, cite each article independently as indicated on the article page online and as indicated below:

LastName, A.A.; LastName, B.B.; LastName, C.C. Article Title. Journal Name Year, Article Number, Page Range.

ISBN 978-3-03928-879-3 (Hbk)

ISBN 978-3-03928-880-9 (PDF)

(C) 2020 by the authors. Articles in this book are Open Access and distributed under the Creative Commons Attribution (CC BY) license, which allows users to download, copy and build upon published articles, as long as the author and publisher are properly credited, which ensures maximum dissemination and a wider impact of our publications.

The book as a whole is distributed by MDPI under the terms and conditions of the Creative Commons license CC BY-NC-ND. 


\section{Contents}

About the Special Issue Editor $\ldots \ldots \ldots \ldots \ldots \ldots \ldots$ vii

\section{Nicola Bellomo, Damian Knopoff and Pietro Terna}

Special Issue "Kinetic Theory and Swarming Tools to Modeling Complex Systems-Symmetry problems in the Science of Living Systems"-Editorial and Research Perspectives

Reprinted from: Symmetry 2020, 12, 456, doi:10.3390/sym12030456

Diletta Burini and Silvana De Lillo

On the Complex Interaction between Collective Learning and Social Dynamics

Reprinted from: Symmetry 2019, 11,967, doi:10.3390/sym11080967 . . . . . . . . . . . . . . . 7

Mirosław Lachowicz, Henryk Leszczyński and Elżbieta Puźniakowska-Gałuch

Diffusive and Anti-Diffusive Behavior for Kinetic Models of Opinion Dynamics

Reprinted from: Symmetry 2019, 11, 1024, doi:10.3390/sym11081024 . . . . . . . . . . . . . 21

Marina Dolfin, Leone Leonida and Eleonora Muzzupappa

Forecasting Efficient Risk/Return Frontier for Equity Risk with a KTAP Approach-A Case

Study in Milan Stock Exchange

Reprinted from: Symmetry 2019, 11, 1055, doi:10.3390/sym11081055 . . . . . . . . . . . . . 37

Damián Knopoff, Juanjo Nieto and Luis Urrutia

Numerical Simulation of a Multiscale Cell Motility Model Based on the Kinetic Theory of

Active Particles

Reprinted from: Symmetry 2019, 11, 1003, doi:10.3390/sym11081003 . . . . . . . . . . . . . . 51

Juan Calvo, Juanjo Nieto and Mohamed Zagour

Kinetic Model for Vehicular Traffic with Continuum Velocity and Mean Field Interactions ${ }^{\dagger}$

Reprinted from: Symmetry 2019, 11, 1093, doi:10.3390/sym11091093 . . . . . . . . . . . . . 71

Ahmed Elaiw, Yusuf Al-Turki and Mohamed Alghamdi

A Critical Analysis of Behavioural Crowd Dynamics-From a Modelling Strategy to Kinetic

Theory Methods

Reprinted from: Symmetry 2019, 11, 851, doi:10.3390/sym11070851 _ . . . . . . . . . . 8

Ahmed Elaiw and Yusuf Al-Turki

Particle Methods Simulations by Kinetic Theory Models of Human Crowds Accounting for Stress Conditions

Reprinted from: Symmetry 2020, 12, 14, doi:10.3390/sym12010014 _ . . . . . . . . . . . . . 97 



\section{About the Special Issue Editor}

Nicola Bellomo Nicola Bellomo is Distinguished Professor at the University of Granada and Professor Emeritus at the Polytechnic University of Torino. He began his career in 1980 when he was called to cover the chair of mathematical physics and applied mathematics due to his scientific achievements on the mathematical theory of the Boltzmann equation and of stochastic differential equations. Subsequently, his scientific interests shifted to the study of living-hence complex-systems, becoming one of the pioneers of the development of active particle methods for the modeling of large systems of self-propelled interacting entities.

NB has published around 200 papers and 8 books devoted to modeling, analytic problems, and simulations of complex systems such as immune competition, vehicular traffic, crowd dynamics, and swarm theory. Thanks to the attention of the scientific community, he has been ranked in the top $1 \%$ of highly cited mathematicians in the world since 2014. In 2009, NB delivered the prestigious Shank Lecture at the Vanderbilt University on the modeling of immune competition. In 2014, he delivered a special lecture in an Oberwolfach workshop on the mathematics of self-propelled particles.

NB is Editor-in-Chief of numerous journals, including Mathematical Models and Methods in Applied Sciences (with Franco Brezzi) and Surveys in Mathematical Sciences (with Simon Salamon); Europe coordinator of two research training networks in 2000-2003 and 2004-2008 on the interaction between mathematics and oncology; Work Package Leader of various EU projects from 2008 to 2017, first on genetic diseases and subsequently on crowd dynamics and social conflicts; Chairman of numerous international conferences, including two Oberwolfach workshops; President of the Italian Society of Industrial and Applied Mathematics 2009-2016; President of the Society of the Italian Highly Cited Scientists. NB has been awarded by the Italian President of the "Third Level Honor" for his scientific contributions. 

Editorial

\title{
Special Issue "Kinetic Theory and Swarming Tools to Modeling Complex Systems-Symmetry problems in the Science of Living Systems"-Editorial and Research Perspectives
}

\author{
Nicola Bellomo ${ }^{1,2,3, *}$, Damián A. Knopoff ${ }^{4}$ and Pietro Terna ${ }^{5}$ \\ 1 Departamento de Matemática Aplicada, University of Granada, 18071 Granada, Spain \\ 2 Department of Mathematical Sciences, Politecnico di Torino, 10100 Torino, Italy \\ 3 IMATI CNR, 27100 Pavia, Italy \\ 4 CIEM CONICET and FaMAF-National University of Cordoba, 5000 Cordoba, Argentina; \\ damian.knopoff@unc.edu.ar \\ 5 School of Management and Economics, University of Torin, 10100 Torino, Italy; pietro.terna@unito.it \\ * Correspondence: nicola.bellomo@polito.it
}

Received: 4 March 2020; Accepted: 9 March 2020; Published: 13 March 2020

check for

\begin{abstract}
This editorial paper presents a special issue devoted to the development of mathematical tools from kinetic and swarms theory to the modeling and simulations of the dynamics of living systems constituted by very many interacting living entities. Applications refer to several fields: collective learning, behavioral economy, multicellular systems, vehicular traffic, and human crowds. A forward look to research perspectives is focused on the conceptual links between swarms methods and the kinetic theory approach.
\end{abstract}

Keywords: kinetic theory; living systems; social dynamics; active particles; learning; social dynamics; pattern formation

\section{Introduction}

This paper presents the conceptual framework of the special issue "Kinetic Theory and Swarming Tools to Modeling Complex Systems-Symmetry problems in the Science of Living Systems" published in the journal Symmetry. The special issue is devoted to report the research activity in the field by means of a selection of scientific articles, where mathematical tools of the kinetic theory and swarms dynamics can contribute to modeling and simulations of living systems. Indeed, it focuses on a fascinating objective which cannot be tackled by the approach of the so-called hard sciences, specifically mathematics without the invention of new mathematical theories.

It is a highly challenging objective. We cannot naively claim that the contents of this issue provide fundamental contributions to the said objective. On the other hand, it can be claimed that the contents witness the ongoing research activity as well as the growing interest of scientists this field requires an interdisciplinary approach.

Essentially, all papers published in the issue technically refer to the so called kinetic theory of active particles, which is a mathematical theory towards the modeling of large systems of interacting living entities [1]. The edited collection of surveys [2] reports about a broad variety of fields of applications from social sciences to the dynamics of vehicular traffic. The key problem of the modeling approach consists in capturing the complexity features of living systems.

Applications have been developed in various fields of life sciences and, more in general, the so-called behavioral sciences where individual behaviors play a key role in the interactions 
and subsequently on the overall dynamics. The survey [3] shows how research activity keeps attracting a growing interest of applied mathematicians and physicists. The very first step of the methodological approach is the representation of the system by a distribution function which defines the probability distribution over the state at the micro scale (individual based) of the interacting entities. Subsequently, the modeling of interactions can be developed according to the specific features of the specific system under consideration, and finally tools of statistical physics are developed to transfer the dynamics of interactions to that of the collective behaviors of the whole system.

It is worth stressing that the specific features of living systems often lead to nonsymmetric interactions, namely, loss of symmetry appears in the dynamics at the low scale. In fact, living entities always apply a selection to the various signals received in the interaction with other entities.

The interested reader can refer to [1] to obtain a picture of the details and of the framework which constitutes the conceptual basis of the mathematical theory. Therefore, we do not add here further details on the methodological approach, but simply mention that interactions present key features which are typical of living systems, namely interactions are nonlocal and nonlinearly additive, collective and often non symmetric. These features are shared also by alternative approaches such as the theory of swarms reviewed in [4] and developed after the celebrated paper by Cucker and Smale [5], while the interest of applied mathematicians and physicists is witnessed in vast literature, e.g., [6-8] including papers specifically related to economic problems $[9,10]$. In addition, we mention with the aim of providing a fully detailed framework the kinetic theory approach developed by mean field and Fokker-Plank models [11], where a variety of interesting models mainly on social dynamics are reported and implemented by sharp numerical tools.

This editorial note is not limited to report about the contents, but it aims also to develop a forward look to research perspectives somehow motivated by the contents of the issue. The presentation of the contents given in Section 2 is followed by a forward look to research perspectives which is proposed in Section 3. In more details, the outline of the research perspectives avoids being generic and focuses on a well defined topic, namely the link between swarms and kinetic approaches somehow related to the development by discrete variables methods. This topic is treated within a multiscale vision somehow inspired by the sixth Hilbert problem [12].

\section{On the Contents of the Special Issue}

Let us now present the contents of the issue following a sequence somehow related to the rationale of the modeling approach, where each paper is related to the conceptual framework and some key literature related to it.

- A new approach to collective learning is proposed in [13] following previous contributions of the authors, where they developed an approach based on the kinetic theory of active particles [14,15]. The novel contents proposed in [13] refer to the specialization of the different types of learning, which is proposed in the fist part of the paper, and the study of the interactions between collective learning and different types of dynamics, which appears in almost all behavioral phenomena, for instance social conflicts related to welfare strategies $[16,17]$. Learning dynamics is a key feature of the various dynamics treated in the papers cited in the following.

- A model of opinion dynamics is studied in [18], where a sharp asymptotic analysis shows how kinetic type models lead to diffusion problems. This paper refers to a topic which has been widely studied by the kinetic theory approach, for instance [19-21], where learning dynamics is the first step of the complex process leading to opinion formation.

- A contribution to behavioral economy is given in [22]. The authors specifically refer to the approach of the kinetic theory for active particles [23-25] which appears to be effective in capturing the main features of behavioral economy [26,27]. Heterogeneity, up to unethical behaviors [28-30], and interactions between economy and social sciences are fundamental aspects of the mathematical approach to behavioral economy. 
- The biology of cells, in particular the immune competition, has been one of the very first fields of application of kinetic theory methods [31]. Motivations to account for the specific features of cells, to be viewed as a living system, have been frequently posed to mathematicians and physicists by biologists, as shown by the celebrated paper by the Nobel Laureate Leland Hartwell [32] who, focusing on biological systems, indicates some important features which distinguish living systems from the inert matter. Indeed, research hints look at a new biology for this century [33]. The dynamics of cell motion is treated in [34], where authors account for structure of the extracellular matrix, considering cell membrane reactions, haptotaxis and chemotaxis. The modeling is performed at a microscopic scale, while a macroscopic model is derived by a scaling limit.

- The kinetic theory approach to vehicular traffic was initiated by the visionary idea of Prigogine [35]. An interesting contribution to our special issue has been delivered in [36] for models where the microscopic state includes, in addition to position and velocity, also an additional variable deemed to describe the quality of the driver-vehicle micro-system. An additional novelty of this paper is that both short-range and mean field interactions are introduced to depict velocity changes related to passing phenomena in view of modeling the role of toll gates or traffic highlights.

- Two papers have been published on modeling and simulation of the dynamics of human crowds. The first one [37] motivates the kinetic theory approach as the most appropriate scale to describe the dynamics of human crowds. Indeed, the authors show that models at this scale have the ability to capture several features of human crowds, for instance subdivision into different groups pursuing different walking strategies, heterogeneous distribution of the walking ability, interaction between emotional states and walking strategies. The second paper [38], in turn, tackles the problem of simulating the dynamics of human crowds under stress conditions in venues with internal obstacles. Applied mathematicians have devoted a great deal of energy to this research topics which has an impact on safety problems and require advanced mathematical tools as witnessed in the very recent literature, see [4,11,39-43]. The authors account for the pertinent literature and develop simulations in a geometry somehow inspired to that of Jamarat bridge. Montecarlo particle methods [44,45] have been used to develop simulations. The application of this computational approach is not straightforward due to the presence of the activity variable, on the other hand it is the most appropriate to account for the specific stochastic feature of kinetic models.

\section{On a Forward Look to Research Perspectives}

The interesting contributions to this special issue motivate some reasonings on research perspectives. However, rather than producing a list of open problems, we will focus this section on a specific research perspective, namely, a multiscale vision of living systems. This topic has been selected, out of various possible ones, according to the idea that only multiscale methods can lead to a consistent description of living systems. Indeed, this is the visionary concept, posed to the attention of mathematicians and physicists, in the Sixth Hilbert problem [12].

The reference scale, which this special issue refers to, is the mesoscale based on kinetic theory methods which has been applied by all the papers herein reviewed. It is possible, according to the methodological approach proposed in [46,47], to derive models at the macroscopic scale from the underlying description at the microscale. Some examples are available in the literature concerning vehicular traffic [48], crowd dynamics [49], and multicellular systems [50,51].

On the other hand, a key problem, related to the selection of the representation and modeling scale, is the validity of the continuity assumption of the probability distribution. Indeed, this assumption is consistent only if the number of interacting a-particles is high enough. Arguably, the number of interacting entities is not large enough to justify the aforementioned assumption. Hence, a research perspective consists in looking for dynamical systems with finite degrees of freedom suitable to model systems at the lower scale. 
This objective requires the derivation of a mathematical framework accounting for the specific features of the interaction dynamics, namely nonlinear additivity and nonlocal dynamics. A first step towards this specific objective has been proposed in [52], referring to a large system of active particles subdivided into functional subsystems. In more detail, the following general framework has been proposed:

$$
\left\{\begin{array}{l}
\frac{d u_{i j}}{d t}=v_{i j}, \\
\frac{d v_{i j}}{d t}=\sum_{p=1}^{n} \sum_{q=1}^{N_{p}} \eta_{i j}^{p q}(\mathbf{u}, \mathbf{v}) \varphi_{i j}^{p q}(\mathbf{u}, \mathbf{v})+\sum_{p=1}^{n} \mu_{i j}^{p}\left(\mathbf{u}, \mathbb{E}_{p}\right) \psi_{i j}^{p}\left(\mathbf{u}, \mathbb{E}_{p}\right),
\end{array}\right.
$$

where the subscript $i, j$ identify, respectively, the $i$-th functional subsystem and the $j$-th a-particle, while $N_{i}$ denotes the number of particles in the i-th FS, and $n$ the number of FSs. In addition:

$\eta_{i j}^{p q}$ models the interaction rate of individual based interactions between $i j$-particles and $p q$-particles; $\mu_{i j}^{p}$ models the micro-macro interaction rate between $i j$-particles and $p$-functional subsystem; $\varphi_{i j}^{p q}$ denotes the micro-micro action, which occurs with rate $\eta_{i j}^{p q}$, of an $p q$-particle over an $i j$-particle; $\psi_{i j}^{p}$ denotes the micro-macro action, which occurs with rate $\mu_{i j}^{p}$ of a $p$-functional subsystem over an $i j$-particle.

The mathematical structure is obtained modeling the action given by the product $\eta \varphi$ for the micro-micro interactions and by $\mu \psi$ for the micro-macro interactions and by equating the overall action to the acceleration of the variable $u_{i j}$, where $\mathbf{u}=\mathbf{u}(t)$ and $\mathbf{v}=\mathbf{v}(t)$ denote, respectively, the set of all states $u_{i j}$ and speed of growth $v_{i j}$. Mathematical models are obtained by implementing the structure (1) with the description of individual based interactions. The application studied in [52] refers to modeling the dynamics of prices due to the interactions between producers-sellers and buyers.

It is useful observing that the behavioral swarm method can be viewed as a generalized mathematical formalization of the Agent-Based Model (ABM) field. In an ABM perspective [53-55] we have simple of complicated agents, with internal rules, interacting one to one or in groups or with institutions. The starting point is always a population of agents, representing individuals or more generally entities, as the component of a generic system, that we construct using small parts of computer code operating in dedicated software environments. The goal is to search for regularities at the macro level generated by the behavior of the agents (micro level, if individuals; meso level, if more complex entities).

In ABMs, we are close to swarms, but operating in a very different manner. The mathematical generalization requires limitations. As a consequence, we can develop our research in a double way, with both a swarm construction and an agent-based one. The first one can be guided by strict formal limitations, requiring to have a more in-depth look at the way the agents behave; the second one offers interesting verifications that we can obtain relaxing some of the constraints introduced in the swarm model. However, the behavioral swarm method provides a more general and flexible approach worth to be further developed towards applications in behavioral sciences [3].

Funding: This research received no external funding.

Conflicts of Interest: The authors declare no conflict of interest.

\section{References}

1. Bellomo, N.; Bellouquid, A.; Gibelli, L.; Outada, N. A Quest Towards a Mathematical Theory of Living Systems; Birkhäuser: New York, NY, USA, 2017.

2. Ball, P. Why Society is a Complex Matter; Springer: Heidelberg, Germany, 2012.

3. Kwon, H.R.; Silva, E.A. Mapping the Landscape of Behavioral Theories: Systematic Literature Review. J. Plan. Lit. 2019. [CrossRef] 
4. Albi, G.; Bellomo, N.; Fermo, L.; Ha, S.-Y.; Kim, J.; Pareschi, L.; Poyato, D.; Soler, J. Traffic, crowds, and swarms. From kinetic theory and multiscale methods to applications and research perspectives. Math. Model. Methods Appl. Sci. 2019, 29, 1901-2005. [CrossRef]

5. Cucker, F; Smale, S. Emergent behavior in flocks. IEEE Trans. Automat. Contr. 2007, 52, 853-862.

6. Bellomo, N.; Ha, S.-Y. A quest toward a mathematical theory of the dynamics of swarms. Math. Model. Methods Appl. Sci. 2017, 27, 745-770. [CrossRef]

7. Ha, S.-Y.; Kim, J.; Ruggeri, T. Emergent behaviors of thermodynamic Cucker-Smale particles. SIAM J. Math. Anal. 2018, 50, 3092-3121. [CrossRef]

8. Fang, D.; Ha, S.-Y.; Jin, S. Emergent behaviors of the Cucker-Smale ensemble under attractive-repulsive couplings and Rayleigh frictions. Math. Model. Methods Appl. Sci. 2019, 19, 1349-1385. [CrossRef]

9. Ahn, S.-M.; Bae, H.-O.; Seung, S.-Y.; Kim, Y.; Lim, H. Application of flocking mechanisms, to the modeling of stochastic volatily. Math. Models Methods Appl. Sci. 2013, 23, 1603-1628. [CrossRef]

10. Bae, H.-O.; Cho, S.-Y.; Kim, J.; Yun, S.-B. A kinetic description for the herding behavior in financial market. J. Stat. Phys. 2019, 176, 398-424. [CrossRef]

11. Pareschi, L.; Toscani, G. Interacting Multiagent Systems: Kinetic Equations and Monte Carlo Methods; Oxford University Press: Oxford, UK, 2013.

12. Hilbert, D. Mathematical problems. Bull. Am. Math. Soc. 1902, 8, 437-479. [CrossRef]

13. Burini, D.; De Lillo, S. On the complex interaction between collective learning and social dynamics. Symmetry 2019, 11, 967. [CrossRef]

14. Burini, D.; De Lillo, S.; Gibelli, L. Collective learning modeling based on the kinetic theory of active particles. Phys. Life Rev. 2016, 16, 126-139. [CrossRef] [PubMed]

15. Burini, D.; Gibelli, L.; Outada, N. A kinetic theory approach to the modeling of complex living systems. In Active Particles, Volume 1; Series: Modelling Simulations Science Engineering Technology; Springer: Berlin, Germany, 2017; pp. 229-258.

16. Bellomo, N.; Herrero, M.A.; Tosin, A. On the dynamics of social conflicts looking for the Black Swan. Kinet. Relat. Models 2013, 6, 459-479. [CrossRef]

17. Furioli, G.; Pulvirenti, A.; Terraneo, E.; Toscani, G. Fokker-Planck equations in the modeling of socio-economic phenomena. Math. Mod. Meth. Appl. Sci. 2017, 27, 115-158. [CrossRef]

18. Lachowicz, M.; Leszczyński, H.; Puźniakowska-Galuch, E. Diffusive and anti-diffusive behavior for kinetic models of opinion dynamics. Symmetry 2019, 11, 1024. [CrossRef]

19. Dolfin, D.; Lachowicz, M. Modeling opinion dynamics: How the network enhances consensus. Netw. Heterog. Media 2015, 4, 877-896. [CrossRef]

20. Knopoff, D. On the modeling of migration phenomena on small networks. Math. Mod. Meth. Appl. Sci. 2013, 23, 541-563. [CrossRef]

21. Knopoff, D. On a mathematical theory of complex systems on networks with application to opinion formation. Math. Model. Methods Appl. Sci. 2014, 24, 405-426. [CrossRef]

22. Dolfin, M.; Leonida, L.; Muzzupappa, E. Forecasting Efficient Risk/Return Frontier for Equity Risk with a KTAP Approach: Case Study in Milan Stock Exchange. Symmetry 2019, 11, 1055. [CrossRef]

23. Ajmone Marsan, G.; Bellomo, N.; Gibelli, L. Stochastic evolutionary differential games toward a systems theory of behavioral social dynamics. Math. Model. Methods Appl. Sci. 2016, 26, 1051-1093. [CrossRef]

24. Bellomo, N.; Colasuonno, F.; Knopoff, D.; Soler, J. From a systems theory of sociology to modeling the onset and evolution of criminality. Netw. Heterog. Media 2015, 10, 421-441. [CrossRef]

25. Dolfin, M.; Knopoff, D.; Leonida, L.; Patti, D. Escaping the trap of "blocking": A kinetic model linking economic development and political competition. Kinet. Relat. Model. 2017, 10, 423-443. [CrossRef]

26. Thaler, R.H.; Sunstein, C. Nudge: Improving Decisions About Health, Wealth, and Happiness; Penguin: New York, NY, USA, 2016.

27. Thaler, R.H. Behavioral Economics: Past, Present, and Future. Am. Econ. Rev. 2016, 106, 1577-1600. [CrossRef]

28. Piff, P.K.; Stancato, D.M.; Coté, S.; Mendoza-Denton, R.; Keltner, D. Higher social class predicts increased unethical behavior. Proc. Natl. Acad. Sci. USA 2014, 109, 4086-4091. [CrossRef] [PubMed]

29. Salvi, S. Corruption corrupts: Society-level rule violations affect individuals' intrinsic honesty. Nature 2016, 531, 456-457. [CrossRef] [PubMed]

30. Liu, L.; Chen, X.; Szolnoki, A. Evolutionary dynamics of cooperation in a population with probabilistic corrupt enforcers and violators. Math. Model. Methods Appl. Sci. 2018, 29, 2127-2149. [CrossRef] 
31. Bellouquid, A.; Delitala, M. Modelling Complex Biological Systems-A Kinetic Theory Approach. In Modeling and Simulation in Science, Engineering and Technology; Birkhäuser: Boston, MA, USA, 2006.

32. Hartwell, H.L.; Hopfield, J.J.; Leibler, S.; Murray, A.W. From molecular to modular cell biology. Nature 1999, 402, c47-c52. [CrossRef]

33. Woese, C.R. A new biology for a new century. Microbiol. Mol. Biol. Rev. 2004, 68, 173-186. [CrossRef]

34. Knopoff, D.; Nieto, J.; Urrutia, L. Numerical simulation of a multiscale cell motility model based on the kinetic theory of active particles. Symmetry 2019, 11, 1003. [CrossRef]

35. Prigogine, I.; Herman, R. Kinetic Theory of Vehicular Traffic; Elsevier: New York, NY, USA, 1971.

36. Calvo, J.; Nieto, J.; Zagour, M. Kinetic Model for Vehicular Traffic with Continuum Velocity and Mean Field Interactions. Symmetry 2019, 11, 1093. [CrossRef]

37. Elaiw, A.; Al-Turki, Y.; Alghamdi, M. A critical analysis of behavioural crowd dynamics: From a modelling strategy to kinetic theory methods. Symmetry 2019, 11, 851. [CrossRef]

38. Elaiw, A.; Al-Turki, Y. Particle methods simulations by kinetic theory models of human crowds accounting for stress conditions. Symmetry 2020, 12, 14. [CrossRef]

39. Aylaj, B.; Bellomo, N.; Gibelli, L.; Reali, A. On a unified multiscale vision of behavioral crowds. Math. Model. Methods Appl. Sci. 2020, 30, 1-22. [CrossRef]

40. Bellomo, N.; Gibelli, L.; Outada, N. On the interplay between behavioral dynamics and social interactions in human crowds. Kinet. Relat. Model. 2019, 12, 397-409. [CrossRef]

41. Bailo, R.; Carrillo, J.A.; Degond, P. Pedestrian models based on rational behaviour. In Crowd Dynamics, Volume 1-Theory, Models, and Safety Problems; Modeling and Simulation in Science, Engineering, and Technology; Birkhäuser: New York, NY, USA, 2018.

42. Goldsztein, G.H. Self-Organization When Pedestrians Move in Opposite Directions. Multi-Lane Circular Track Model. Appl. Sci. 2020, 10, 563. [CrossRef]

43. Kim, D.; Quaini, A. A kinetic theory approach to model pedestrian dynamics in bounded domains with obstacles. Kinet. Relat. Model. 2019, 12, 1273-1296. [CrossRef]

44. Aristov, V.V. Biological systems as nonequilibrium structures described by kinetic methods. Results Phys. 2019, 13, 102232. [CrossRef]

45. Barbante, P.; Frezzotti, A.; Gibelli, L. A kinetic theory description of liquid menisci at the microscale. Kinet. Relat. Model. 2015, 8, 235-254.

46. Burini, D.; Chouhad, N. Hilbert method toward a multiscale analysis from kinetic to macroscopic models for active particles. Math. Model. Methods Appl. Sci. 2017, 27, 1327-1353. [CrossRef]

47. Burini, D.; Chouhad, N. A Multiscale view of nonlinear diffusion in biology: From cells to tissues. Math. Model. Methods Appl. Sci. 2019, 29, 791-823. [CrossRef]

48. Bellomo, N.; Bellouquid, A.; Nieto, J.; Soler, J. On the multiscale modeling of vehicular traffic: From kinetic to hydrodynamics. Discret. Cont. Dyn. B 2014, 19, 1869-1888. [CrossRef]

49. Bellomo, N.; Bellouquid, A. On multiscale models of pedestrian crowds from mesoscopic to macroscopic. Commun. Math. Sci. 2015, 13, 1649-1664. [CrossRef]

50. Bellomo, N.; Bellouquid, A.; Nieto, J.; Soler, J. On the asymptotic theory from microscopic to macroscopic growing tissue models: An overview with perspectives. Math. Model. Methods Appl. Sci. 2012, 22. [CrossRef]

51. Bellomo, N.; Bellouquid, A.; Chouhad, N. From a multiscale derivation of nonlinear cross-diffusion models to Keller-Segel models in a Navier-Stokes fluid. Math. Model. Methods Appl. Sci. 2016, 26, 2041-2069. [CrossRef]

52. Bellomo, N.; De Nigris, S.; Knopoff, D.; Morini, M.; Terna, P. Swarms dynamics towards a systems approach to social sciences and behavioral economy. Netw. Heterog. Media 2020, in press.

53. Gilbert, N.; Terna, P. How to build and use agent-based models in social science. Mind Soc. 2000, 1, 57-72. [CrossRef]

54. Tesfatsion, L. Agent-based computational economics: Modeling economies as complex adaptive systems. Inf. Sci. 2003, 149, 262-268. [CrossRef]

55. Grimm, V.; Railsback, S.F.; Vincenot, C.E.; Berger, U.; Gallagher, C.; DeAngelis, D.L.; Edmonds, B.; Ge, J.; Giske, J.; Groeneveld, J.; et al. The odd protocol for describing agent-based and other simulation models: A second update to improve clarity, replication, and structural realism. J. Artif. Soc. Simul. 2020, $23,7$.

(C) 2020 by the authors. Licensee MDPI, Basel, Switzerland. This article is an open access article distributed under the terms and conditions of the Creative Commons Attribution (CC BY) license (http:/ / creativecommons.org/licenses/by/4.0/). 
Article

\title{
On the Complex Interaction between Collective Learning and Social Dynamics
}

\author{
Diletta Burini ${ }^{1, *,+}$ and Silvana De Lillo ${ }^{1,2,+}$ \\ 1 Department of Mathematics and Computer Science, University of Perugia, 06123 Perugia, Italy \\ 2 Istituto Nazionale di Fisica Nucleare, 06123 Sezione di Perugia, Italy \\ * Correspondence: dilettaburini@alice.it \\ + These authors contributed equally to this work.
}

Received: 28 June 2019; Accepted: 22 July 2019; Published: 1 August 2019

check for updates

\begin{abstract}
This paper is motivated by the perspective ideas proposed in our previous studies, where some challenging problems, for instance qualitative analysis of the solution to nonlinear problems and micro-macro asymptotic analysis, where posed. Our work focuses on the study of the interactions between learning dynamics and other types of dynamics which can be modeled by kinetic theory methods. The contents are presented in three parts. First, a general description of different theories of learning dynamics within the framework of cognitive sciences is critically analyzed with the aim of capturing the main features of the system towards modeling. Subsequently, the class of systems which are the object of the modeling approach is defined by showing how the previous structure can be developed, thanks to new conceptual ideas, including the concept of symmetric and asymmetric learning, towards modeling. Finally, some applications are selected to show how the approach can be methodologically applied.
\end{abstract}

Keywords: learning dynamics; kinetic theory; complex systems

\section{Plan of the Paper}

A mathematical approach to the collective learning dynamics has been developed in [1-3] to model individual and collective learning by interactions involving individuals and groups of individuals. The method combines the mathematical tools of the kinetic theory of active particles [4] and the theoretical tools of evolutionary game theory [5,6]. In more detail, the sequential steps of the approach are as follows:

1. The level of learning of a certain knowledge is modeled, at the microscopic level, by a scalar variable $u \in[0,1]$, where $u=0$ represents the lowest level of achieved knowledge and $u=1$ the highest admissible level.

2. The whole system can be subdivided into functional subsystems, in which each one of them plays a different role in the learning process.

3. The overall state of the system is delivered, i.e., a probability distribution function over the microscopic state defines the collective state of the system, while macroscopic states are provided by weighted moments of this probability distribution.

4. The dynamics develops through encounters which can be either individual-based, or between individuals and the whole system.

5. The output of the interactions is modeled by developments of the theoretical tools of the evolutionary game theory, in which players are probability distributions or their averaged quantities. 
6. The probability distribution, mentioned in Item 3 , is the dependent variable of a differential system. Its dynamics is obtained by a balance of "living" particles in the elementary volume of the microscopic states.

The interested reader is referred to [1] for additional information on the modeling approach, to the survey [7] and to the book [4] for the related mathematical tools, while additional details will be given in the next sections.

The contents of our paper rely on the observation that the perspective ideas proposed in [1] leave open various problems that are definitely worth of future investigations. In more detail, this present paper focuses on the study of the interactions between learning dynamics and other types of dynamics that can be accounted for by kinetic theory tools. In fact, some recent papers have put in evidence how models which involve interactions of different dynamics can generate even outputs that could not be foreseen, but which are confirmed in reality.

A possible example is given in [8], where it is shown that an unfair wealth policy can lead to a radicalization of the opposition to the governments which has produced such policy. The opposition also ends up involving the social classes that apparently take advantage of the policy, however unfair it is. Further applications are suggested in [9], focusing on the interactions between learning and behavioral economy. An additional example is the study of the complex interaction between learning in crowd dynamics, in which the walking strategy is induced by learning social-emotional behaviors.

These examples, discussed in the last section, motivate the contents of this paper which is devoted to the modeling of the influence of learning dynamics on other types of dynamics. This is the main novelty of our paper, where, in addition, the use of discrete states of learning dynamics and of the variables of subsequent dynamics is developed, also exploiting a new metrics to model the distance between the interacting entities. The contents are proposed through three other sections.

Section 2 provides a general description of the different theories of learning dynamics in the framework of cognitive sciences. Some specific issues are extracted, without claim of completeness, from a vast and heterogeneous literature that is still waiting for a unified interpretation. This section attempts to open a dialogue between mathematics and cognitive sciences looking forward to a unified approach generated by the mathematical sciences.

Section 3 defines the specific systems to be modeled and then shows how the approach proposed in [1] can be developed, thanks to new conceptual approaches, towards the modeling of the class of systems presented in Section 2. The conceptual novelties consist of using the discrete probability distribution to describe the state of the system and the discrete stochastic games to model the interactions, and in dealing with a hierarchy where learning precedes a consequent dynamics.

Section 4 deals with applications, selected among several possible ones, to show how the approach can be specifically referred to modeling. These applications specifically refer to the two aforementioned dynamics, firstly the modeling of wealth policy opposition to governments up to radicalization and then the interaction between learning walking strategy in crowd dynamics. Lastly, we consider a third application i.e., the immune competition in heterogenous multicellular systems, where learning leads to proliferative and destructive events. The presentation of these case studies concludes with a critical analysis that, starting from the theoretical achievements of our paper, looks ahead to research perspectives.

\section{State of the Art}

This section introduces topics related to collective learning that constitute the basis for the modeling approach developed in our paper. The contents are presented in three subsections. The phenomenological description of collective learning is treated in the first subsection. An overview of the existing mathematical approaches known in the literature is delivered in the second subsection. The third subsection provides a critical analysis that defines more precisely the objectives of the mathematical method proposed from Section 3. 


\subsection{Phenomenological Description of Collective Learning}

We start examining the interrelations between individual and social learning, which appear as two different aspects of the same phenomenon of collective learning, to clarify how they are interconnected.

The concept of individual learning has come to be embedded into social and cultural contexts and interactions, due to the growing interest in Vygotsky's theory [10], to retrospective examinations of the role of social interactions in [11], and in the subsequent Bandura's revisiting [12]. Two strands are involved: the "cognitive, acquisition-oriented" conception of individual learning with respect to the "situative, participatory" concept [13]. Learning occurs in individuals' minds and, as we elaborate later, it takes place as a social and participatory process, offering two distinctively different points of view on learning.

The idea of social learning originated with the development of psychology sciences [14] and occurs when the individual learns new behaviors and concepts from others. Because of its lack of rigor, it has been ignored by psychologists over the years, and it has been relegated, at best, to the study of the background context, not exactly on a par with the learning of the individual [15]. Social learning is often combined with other dynamics, for instance participation and pro-environmental behavior, while, in general, small differences are referred to individual and social learning.

After this general presentation, we now provide, referring to [16], some characterizations of social learning that can be useful for the modeling process that we want to develop later. The first aspect to keep into account is the active social mediation of individual learning: a person or a team helps an individual to learn e.g., a teacher and a child forming a joint learning system.

Another important characterization, which deserves to be mentioned, consists of considering the social entity as a learning system. A good example is the case of a collective agency that, as a collective, acquires more knowledge, understanding, or skill e.g., a business organization develops internal procedures that satisfy the efficiency of customer requests.

Finally, the last aspect we wish to point out is learning to be a social learner: an important dimension of learning to learn is how that participate in and capitalize on the social milieu.

Over the years, a frequent confusion has emerged between the concept of social learning itself and its potential outcomes. The lack of clarity on this matter has reduced the possibility to distinguish precisely the two, however different, types of learning [17]. For these reasons, in [18], the authors try to answer the question: what is social learning?, and they identify three key problems with the term as it is currently used in the literature:

- Social learning can possibly occur even without any previously organized interaction, while social networks can contribute to the interaction dynamics [19-21].

- $\quad$ The quality, namely level of depth, of this type of learning can span from superficial levels to deep conceptual levels which can involve groups at different scales [22,23].

In conclusion, social learning is effective if a change in understanding appears in the individuals involved in the dynamics.

Closely connected with the previous analysis is the concept of collective learning which is a complex dynamics that has been variously defined in different ways. It is mainly viewed as a dynamic and cumulative process.

An interesting example is provided by the deep learning algorithms recently tested in the diagnosis of skin cancers [24]. The algorithm combines visual processing with deep learning, a type of artificial intelligence modeled by models of neural networks. Learning is then an evolutionary process related to the dynamics of collective knowledge [25].

Among the characterizations of the collective learning, we want to bring the analysis done in the context of transfer knowledge in technology milieu, where in [26] the author approaches a definition of collective learning and its interpretation within spatial theories. First, collective learning to be interpreted as a social process of cumulative knowledge $[27,28]$. 
In conclusion, if learning is to be considered collective learning, then it must be cumulative and interactive. Learning is a dynamic process, developed on the basis of an element of continuity over time, on which knowledge rests and accumulates. Furthermore, the new knowledge of a first inventor is transferred through other agents on the basis of an element of synergy and an interactive process, giving rise to a cumulative process of knowledge creation.

\subsection{A Brief Survey of Mathematical Approaches}

Due to the lack of a socio-psychological theory on social learning, mathematical approaches also differ significantly from one another, waiting for a possible uniformity. In this subsection, we present some approaches, bearing in mind the three sequential steps that occur in the rough simplification of learning dynamics in complex living systems toward modeling. The first step of learning dynamics is the perception of inputs from other individuals. These inputs are perceived within an individual domain within which the said inputs are effectively felt. Thus, interactions take place and trigger a learning process that changes their level of knowledge.

From the point of view of theoretical modeling, very interesting studies have been carried out in the framework of statistical physics methods in recent years. A very broad review concerning this general framework was presented in [29], where the authors focus their attention on different aspects emerging in social dynamics. All such aspects seem to be related to the fundamental issue of how the interactions between social agents allow the emergence of a macroscopic order starting from a disordered initial situation. In [29], the authors focus on some conceptually homogeneous topics, sharing the common aspect according to which individuals are viewed as adaptive rather than rational agents that communicate and interact with each other.

In the context of social dynamics, the agreement is one of its most important aspects. Everyday life presents many situations in which it is necessary for a group to reach shared decisions. One of the pioneer contributions to the modeling agreement/disagreement dynamics, which might be referred to opinion dynamics, has been proposed by a model proposed by Weidlich [30]. Subsequently, a modification of the Ising model has been proposed to model specific features of opinion dynamics [31,32], where the spin-spin coupling is deemed to model pairwise interaction, while a background field represented the cultural majority or propaganda. Subsequently, a branch of opinion dynamic is based on the so-called "voter model" [33], which was first considered in [34] as a model for species competition. Kinetic models of continuous opinion formation involving both the exchange of opinions between individual agents and dissemination of information are proposed in [35-37].

In the framework of stochastic models, the Axelrod model played a prominent role [38], by enlightening the two mechanisms, which, according to his theory, are fundamental in understanding of the dynamics of cultural assimilation, namely social influence and homophily.

Collective learning, on the other hand, has a very strong influence also in the modeling of collective motion. This latter appears in nature with common effects, e.g., observation of flocks of birds and swarms of insects motivated several studies in the past $[39,40]$. Humans display similar behavior in many instances, such as pedestrian motion, panic and vehicular traffic. Pedestrian behavior has been empirically studied since the 1950s [41]. The first physical modeling was proposed in [42] by a comparison with Navier-Stokes equations, but already in [25] it has been observed that in the dynamics of living entities energy and momentum are not generally conserved.

In more recent years, a new approach to behavioral-social dynamics for pedestrian crowds in the framework of the kinetic theory of active particles has been developed by dealing with the modeling of crowd dynamics to show that collective behaviors are induced by individual bases interactions $[43,44]$. The learning dynamics have an important role in crowd models as the walking strategy takes advantage of what each individual learns from the other walkers [45]. In this approach, the level of stress is not simply modeled as a parameter, but it is a variable whose dynamics is related to learning and social contagion. 


\subsection{Critical Analysis}

The mathematical modeling of collective learning should provide a characterization of all the fundamental aspects involved in the process and at the same time to be able to capture the fundamental aspects that determine the dynamical evolution of the system. The main feature to tackle is the complexity of the living systems. Indeed, the phenomenon we wish to describe can be represented considering a large collection of living entities which interact among them at microscopic level, through nonlinear interactions. The outcome of all the interactions determines the dynamical evolution of the overall system at a macroscopic level.

The aim of the present paper is to construct a mathematical framework suitable to capture the following aspects of the learning process:

- The individual's role in the collectivity. Collective learning modifies the individual's ability to develop interaction rules that evolve over time.

- Learning dynamics. Living entities can learn not only by micro-scale interactions, but also from the whole population. The key problem consists of referring the dynamics at a micro-scale to treat at the macro-scale.

- Role of networks. Networks can have an important influence on the learning dynamics, as observed in [9] both exogenous and endogenous networks have to be considered corresponding, respectively, to physically localized nodes and nodes generated by aggregations due to affinity principles.

\section{Mathematical Tools}

The derivation of mathematical models to describe the dynamics of collective learning is developed in two consecutive phases. Firstly, by defining a general structure capable of capturing the specific features of the class of systems under examination, and subsequently by creating models related to well defined learning phenomena. The derivation is obtained by refining the general structure by inserting into it models suitable to describe the interactions among the subjects involved in the learning dynamics. Examples of interactions between learning and social dynamics will be analyzed in the next section, while only methodological aspects are treated here.

Further conceptual developments are needed, with respect to [1], because our paper addresses complex dynamics for which learning is an important component, but only a preparatory step preceding other dynamics. In fact, in addition to the variables that characterize the learning process, it is also necessary to include more variables characterizing the induced dynamics.

This brief introduction defines the content of this section that will be dealt with in the following subsections addressed to the main three themes:

1. Characterization of the soft variables and representation of the system;

2. Modeling of interactions;

3. Derivation of a general structure suitable to describe the collective dynamics of interactions between learning and social dynamics.

The study will be limited to the case where the microscopic variable is defined by two components, i.e., learning and social. The methodological approach can then be generalized technically in the case of a larger number of components as discussed in the last section of our paper. Hereafter, we will use the following terminology: active particles (a-particles) to identify interacting entities; activity to indicate their status at the microscopic scale (individual based); and functional subsystems (FSs) with reference to the groups in which the population is divided.

\subsection{Soft Variables and Representation}

We consider a system of a large number of interacting entities with the following features:

- The system is composed by living entities which interact in a spatially homogeneous dynamics; 
- $\quad$ The microscopic scale corresponds to the state of each entity modeled as an a-particle [4];

- $\quad$ Each a-particle interacts with the others at both the microscopic level and the collective set of all the particles.

Therefore, according to [4], we introduce the activity, which is a vector variable with two components $\mathbf{w}=\{u, v\}$, where $u$ is the component that characterizes the level of learning and $v$ is the social component whose dynamics are also induced by $u$. Both $u$ and $v$ can be defined, according to an occasionally used jargon, soft variables.

In principles, although accounting for Sigmund's important book [46], soft variables are not measurable. However, we can conjecture and identify the minimum and maximum values that these variables may reach by defining a suitable interval where values are observed. Therefore, normalizing with respect to that domain it is obtained: $u \in[0,1] \equiv D_{u}$ and $v \in[0,1] \equiv D_{v}$. Moreover, local measures cannot be effectively made, therefore it is convenient using discrete, rather than continuous, variables.

For instance, a discrete, equally spaced collocation, can be used for the components $u$ and $v$ :

$$
C_{u}=\left\{u_{1}=0, \ldots, u_{n}=1\right\}, \quad \text { and } \quad C_{v}=\left\{v_{1}=0, \ldots, v_{n}=1\right\}
$$

Therefore, the representation is delivered by the discrete probability distributions

$$
\mathbf{f}=\left\{f_{i j}(t)=f\left(u_{i}, v_{j} ; t\right)\right\} \text { with } \sum_{i=1}^{n} \sum_{j=1}^{n} f_{i j}(t)=1 \text {. }
$$

If $f_{i j}(t)$ is known, p-order moments are computed by weighted sums:

$$
\mathbb{E}^{p}\{u\}(t)=\frac{1}{n} \sum_{i=1}^{n} \sum_{j=1}^{n} u_{i}^{p} f_{i j}(t) \text { and } \mathbb{E}^{p}\{v\}(t)=\frac{1}{n} \sum_{i=1}^{n} \sum_{j=1}^{n} v_{j}^{p} f_{i j}(t) .
$$

If active particles are subdivided into $m$ FSs, the following notation is used:

$$
\mathbf{f}^{r}=\left\{f_{i j}^{r}(t)=f^{r}\left(u_{i}, v_{j} ; t\right)\right\} \quad r=1, \ldots, m .
$$

Our paper refers to systems where the total number of particles $N$ is constant in time, so that the normalization condition is written as follows:

$$
\sum_{r=1}^{m} \sum_{i=1}^{n} \sum_{j=1}^{n} f_{i j}^{r}(t)=1
$$

where each $f_{i j}^{r}$ is a component of a probability as it has been divided by $N$.

\subsection{Modeling Interactions}

The first step of the modeling of interactions consists of selecting those interactions that play a non-trivial role in the dynamics of the system, while the second step consists of taking into account also a possible hierarchy among the activities $u$ and $v$, and eventually in modeling the output of interactions. The stochastic game theory [4] describes their dynamics by means of the frequency of the interactions and the transition probability of microscopic states of the interacting active particles which pursue a specific payoff consistent with the strategy they are able to develop. Moreover, rather than individual entities chasing their own pay-off, the whole population operates to achieve a collective well-being, where the advantage is shared by everyone. This concept is well expressed by the following quotation from Ref. [5]:

Evolutionary game theory deals with an entire population of players, all programmed to use the same strategy (or type of behavior). Strategies with higher payoff will spread within the population (this can 
be achieved by learning, by copying or inheriting strategies, or even by infection). The payoffs depend on the actions of the co-players and hence on the frequencies of the strategies within the population. Since these frequencies change according to the payoffs, this leads to a feedback loop. The dynamics of this feedback loop is the object of evolutionary game theory.

This subsection shows how interactions can be described by suitable operators, to be subsequently used in the derivation of the mathematical structure we are looking for. According to the kinetic theory of active particles, the dynamics of interactions involve candidate, field, and test active particles. In detail, we consider:

- $\quad$ Test particles of the $r$-FS with microscopic state $u_{i}, v_{j}$ and probability $f_{i j}^{r}(t)$. These particles are assumed to be representative of the whole system.

- $\quad$ Field particles of the $r$-FS with microscopic state $u_{p}, v_{q}$ and probability $f_{p q}^{r}(t)$ which are generic particles for each $r$-FS.

- $\quad$ Candidate particles, of the $k$-FS with microscopic state $u_{h}, v_{k}$ and probability $f_{h k}^{k}(t)$ which are generic particles for each $k$-FS deemed to take, due to interactions, the state of the test particles.

The modeling of learning interactions can be achieved by the interaction rate which describes the number of encounters per unit time, and the transition probability which denotes the probability that a candidate particle takes the state of the test particle due to interaction with field particles.

Dealing with these modeling issues leads straightforwardly to derive the governing evolution equation which regulates the dynamics of the class of systems under consideration. If we consider the simple case of one FS only, a formal expression of the aforementioned terms is as follows:

$\eta_{h k}^{p q}$ is the interaction rate of a $h k$-particle with a $p q$-particle, namely between particles with states $h k$ and $p q$.

$\mu_{h k}$ is the interaction rate of a $h k$-particle with the mean state $\mathbb{E}$ of the whole system.

$\mathcal{A}_{h k}^{p q}(h k \rightarrow i j)$ models the transition of a candidate $h k$-particle into the state of the test $i j$-particle due to the interaction with a field $p q$-particle.

$\mathcal{M}_{h k}(h k \rightarrow i j)$ models the transition of a candidate $h k$-particle into the state of the test $i j$-particle due to the interaction with the mean value within the system.

A balance of particles in the elementary volume of the microscopic state yields:

$$
\begin{aligned}
\frac{d}{d t} f_{i j} & =\sum_{h, k, p=1}^{n} \eta_{h k}^{p q}(\mathbf{f}) \mathcal{A}_{h k}^{p q}(h k \rightarrow i j)(\mathbf{f}) f_{h k} f_{p q}-f_{i j} \sum_{p, q=1}^{n} \eta_{i j}^{p q}(\mathbf{f}) f_{p q} \\
& +\sum_{h, k=1}^{n} \mu_{h k}(\mathbf{f}) \mathcal{M}_{h k}(h k \rightarrow i j)(\mathbf{f}) f_{h k} \mathbb{E}(\mathbf{f})-f_{i j} \mu_{i j}(\mathbf{f}) \mathbb{E}(\mathbf{f})
\end{aligned}
$$

where all interaction terms might depend on $\mathbf{f}$, while the dependent variables are a function of time.

Analogous calculations can be developed if the system is constituted by a number $m$ of interacting functional subsystems:

$$
\begin{aligned}
\frac{d}{d t} f_{i j}^{r}= & \sum_{s=1}^{m} \sum_{h, k, p, q=1}^{n} \eta_{h k}^{p q}(r, s)(\mathbf{f}) \mathcal{A}_{h k}^{p q}(h k \rightarrow i j)(\mathbf{f}) f_{h k}^{r} f_{p q}^{s}-f_{i j}^{r} \sum_{s=1}^{m} \sum_{p, q=1}^{n} \eta_{i j}^{p q}(\mathbf{f}) f_{p q}^{s} \\
& +\sum_{s=1}^{m} \sum_{h, k,=1}^{n} \mu_{i j}^{s}(\mathbf{f}) \mathcal{M}_{h k}^{s}(h k \rightarrow i j)(\mathbf{f}) f_{h k} \mathbb{E}_{s}(\mathbf{f})-f_{i j}^{r} \sum_{s=1}^{m} \mu_{i j}(\mathbf{f}) \mathbb{E}_{s}(\mathbf{f}) .
\end{aligned}
$$

Still following the methodological style of presentation of our paper, some indications can be given to show how a mathematical description of interactions leads to the derivation of the models. Mainly qualitative indications are provided, leaving a detailed formalization to specific models.

Let us indicate how a possible interaction hierarchy can account for the sequential combination of the two different dynamics, namely learning, which corresponds to the variable $u$, and the subsequent 
dynamics corresponding to $v$. We propose, without claiming full generality, an approach grounded on the assumption that dynamics of the learning component $u$ precedes and is independent of the following dynamics, which, however, also depends on $u$. This assumption is not the most general one, but it can cover. The modeling of the interaction rates and of the transition probability can account, specifically, on the said assumption.

Let us now consider the modeling of the interaction rate which requires the definition of different concepts of distance between interacting entities. Some concepts can be given, which can be applied both to the distance between candidate and field particles and to that between test and field particles. The hierarchy indicates that interactions within the same FS can be considered in sequence. Therefore, the aforementioned distance can be considered: The micro-micro distance refers to the individual microscopic states within the same functional subsystems, namely $\left|u_{i}-u_{p}\right|$ and $\left|v_{j}-v_{q}\right|$, where the subscripts $p$ and $q$ refer to the field particles in the same functional subsystem; and the micro-macro distance refers to the interaction between the individual micro-state and the whole FS whose state is defined through a suitable average, for instance the mean value. Then, one has that micro-macro distances are given by $\left|u_{i}-\mathbb{E}^{s}\right|$ and $\left|v_{j}-\mathbb{E}^{s}\right|$. As a consequence, the interaction rate can be modeled by assuming that the interaction rate decays with the distance, between the interacting entities, measured by a specific metrics. In more detail, the metrics can be obtained by an exponential decay with a distances, either micro-micro or micro-macro from a basic value delivered when the distance is equal to zero. Symmetric interactions appear if interaction rates depend on the metrics defined above, while asymmetric interactions can be modeled by introducing a weight function depending on the localization of the nodes.

Consider now the modeling of the transition probability which models the output of the interaction of a-particles, with state $u_{h}, v_{k}$ within the i-th FS due to interaction with other a-particles as well as with the FS as a whole. The aforementioned hierarchy implies that the dynamics of the learning component $u$ precedes and is independent of the dynamics of the social component $v$, whose interaction dynamics, however, depend on $u$. For this reason, the transition probability $\mathcal{A}$ is composed of the contribution of two transition probabilities, namely the first one modeling the change of the level of knowledge of the candidate particle and a second one which accounts for the subsequent dynamics conditioned by the learning level. Therefore, the transition probability can be given as a factorization of the transition probabilities over the discrete variables $u$ and $v$.

The role of exogenous networks can be taken into account by the selection of the functional subsystem to be related to the localization of the nodes as proposed in [47,48]; here, we use discrete probability rather than continuous probability density. The problem of the creation of endogenous networks generated by aggregations, which is one of the key problems posed in [49] cannot be straightforwardly treated by the approach of this paper as it needs additional work.

The specific applications treated in the next section aims at showing how the aforementioned general approach can be particularized towards specific case studies. In addition, it will be shown how the further developments will be motivated by well defined applications. It is worth mentioning that a common feature of all applications is the interaction of learning dynamics with a different type of dynamics which precedes or follows learning. In mode details, three case studies follow-first, in social dynamics, where welfare policy is an important influence over the learning dynamics underlying the opinion formation. Second, collective learning modifies the mechanical rules by which crowds move. Lastly, collective learning in multicellular systems modifies the proliferative and/or destructive interactions in the immune competition.

\section{Case Studies towards Perspectives}

The literature critically analyzed in Section 2 has enlightened a wide class of possible learning dynamics in view of a modeling approach. We do not naively claim that the methodological approach proposed in our paper can cover the aforementioned whole variety. Therefore, we will focus on the main objective of our description, namely the modeling of the interactions between learning dynamics 
and other types of dynamics which can be modeled by kinetic theory methods. This statement can be made more precise by selecting three case studies, which present different features, to show how the approach can be technically developed.

Firstly, we show how the learning dynamics, related to welfare distribution, can lead to a distribution of support/opposition to governments, which, in turn, can even generate radical opposition. Secondly, we refer to the interaction between learning and crowd dynamics, namely between learning which leads to mechanical dynamics, namely walking strategies. Lastly, we consider the immune competition in heterogenous multicellular systems, where learning leads to proliferative and destructive events. Well defined hints will be given for each of them in view of research programs which might take advantage of the suggestions of this section. As we shall see, some applications might require further developments of the approach proposed in Section 3 as we will critically analyze in the last subsection.

\subsection{Social Conflicts and Radical Opposition}

Let us firstly consider the modeling of a dynamics where a welfare policy can lead to social conflicts and radical opposition. In more detail, let us consider the dynamics treated in [8], where a certain welfare policy generates either support or opposition to the Government author of the said policy. We consider an opinion formation process driven by the learning dynamics by which individuals communicate and "learn". Already, the authors in [8] have shown that the modeling approach should account for nonlinearly additive interactions. This feature has been confirmed in some subsequent papers that have developed this pioneer idea, for instance [50-52]. The approach of [8] models the sequential interaction of two dynamics: wealth distribution which has been treated by various authors e.g., $[36,53,54]$ and opinion formation has been introduced in [37] and subsequently treated by various authors, for instance [55,56]. A survey of the literature on this topic has been reviewed in [57].

The modeling of wealth distribution precedes the modeling of support or opposition to a government. Our hint is that the modeling approach developed in [8] deserves a revisiting focused on a deep analysis on the role of learning dynamics. In particular, the following research objectives are proposed:

1. Modeling the role of the individual learning from the whole population to understand how a certain trend, which can be conditioned by the wealth distribution, can lead to radicalization of the political contrast.

2. Modeling the transition, which can be viewed as a Darwinist mutation from a radical, however democratic, opposition, to a segregation of individuals ready to break laws and bring their opposition up to extreme levels.

3. Modeling the selection following mutations by an approach which might require the addition of a new FS corresponding to security forces/actions to act against the aforementioned radicalization.

The learning dynamics presented in [8] were based on simple rules, while the modeling of the mutations towards radicalization can be developed by introducing a threshold in the radicalization level which identifies the transition to extremism.

\subsection{Collective Learning and Dynamics of Crowds}

The second case study refers to the interaction between learning dynamics which produces social behaviors and the dynamics of crowds which is a mechanical system, where human behaviors [44] appear. Learning can have an important influence over the walking strategy of individuals in the crowd as it is shown in a recent paper [45], where a modeling approach includes the propagation of stress conditions. In particular, it is shown how stress conditions can modify both the overall dynamics and density patterns, inducing the formation of overcrowded zones, which is a bad event against safety. The modeling approach proposed in [45] is based on tools of the kinetic theory initiated in [43] 
and further developed in [44], while the derivation of macroscopic equations from the underlying description at the microscopic scale delivered by kinetic models has been obtained [58,59].

This topic deserves further research developments which might take advantage of the following hints:

1. The development of a learning dynamics in a crowd, where individuals communicate and learn through small group awareness. Communications propagate stress, which in turn can generate unsafe dynamics.

2. An additional interesting example is that of swarm's dynamics where communications and learning induce a collective motion with flocking properties [60]. Such specific interaction between learning and mechanics might generate a motion suitable to optimize the swarm defense against the attack of predators [61].

3. Collective learning should include nonlocal effects that have not yet been treated in [1] nor, in full generality, in [45]. Therefore, this research perspective requires further developments of the mathematical theory proposed in Section 3 to account for space dynamics and pattern formation.

\subsection{Multicellular Systems and Immune Competition}

Lastly, let us consider the dynamics of the immune system versus cells carriers of a pathology. We consider, referring to [62], a multicellular system including three populations, namely epithelial, immune, and cancer cells. Each population has a different internal variable. Immune cells can proliferate and activate their internal variable which can correspond to the defence ability against cancer cells. Cancer cells can proliferate and progress towards states with higher pathological states, namely by increasing their proliferative ability up to metastatic competence $[63,64]$. Immune cells have the ability of depleting cancer cells, which, in opposition, can inhibit the defence activity of immune cells. The first learning process of the immune cells is the acquiring of immune competence that enriches the innate immunity [65].

These complex dynamics have been studied in $[62,66,67]$ which have been inspired by researchers in the field of immunology [68] who motivated their conjecture by the idea that learning dynamics can induce mutations both for cancer and immune cells. Subsequently, the dynamics of competition, which ends up with proliferative and destructive events, induces selection. In particular, biologists argue that the learning dynamics occurs not only across the competing species, but also within the same population. For instance, active immune cells can learn from inhibited immune cells, and similarly weakly progressing cancer cells can learn from highly progressing cancer cells.

The following hints are brought to the attention of the interested reader:

1. A possible research program would be a deep analysis of the learning dynamics and the related influence over the immune competition.

2. The approach with an additional difficulty consists of the modeling of a system with a variable number of interacting living entities as proliferative and/or destructive encounters that appear in this dynamics.

3. The number of interacting functional subsystem grows in time due to the onset, by mutations, of new subsystems.

The mathematical tools proposed in Section 3 appear to be consistent with the requirements of the first hint, while the second and third hint need developments of new theoretical tools.

\subsection{Critical Analysis towards Perspectives}

Our paper has shown how the kinetic theory approach to collective learning can be developed to model cross interactions between learning and subsequent dynamics. Three specific applications have been outlined focusing on different living systems, while possible hints have been given to develop these applications. Therefore, looking at research perspectives, it is quite natural to 
tackle the challenging problems presented in this section by taking advantage of the said hints. These applications indicate that the mathematical structures derived in Section 3 might need to be further developed to account for some aspects of the Darwinist dynamics, space propagation and proliferative/destructive events.

In addition to the above reasoning, it is worth mentioning that the approach cannot be limited to modeling, but it should also require development of computational tools. Considering that the approach refers to equations of the kinetic theory, Monte Carlo particle methods appear to be the approach consistent with the structures of these types of equations. Therefore, one has to deal with the development of pioneering works, for instance referring to the books $[69,70]$ to recent applications reported in $[36,71,72]$. Numerical simulations can also contribute to cover, however at a heuristic level, issues that are not easily tractable, such as existence of equilibrium configuration and related stability analysis.

Lastly, let us focus on the problem posed by a multiscale vision, where macro-scale models are derived from micro-scale models as delivered by kinetic type models. A unified approach to this specific multiscale problem has been proposed in [73,74]. It is a general analytic tool that can be further specialized to a micro-macro derivation for the class of systems treated in our paper.

Author Contributions: Conceptualization, D.B. and S.D.L.; Methodology, D.B. and S.D.L.; Writing-original draft, D.B.

Funding: This research received no external funding.

Conflicts of Interest: The authors declare no conflict of interest.

\section{References}

1. Burini, D.; De Lillo, S.; Gibelli, L. Collective learning modeling based on the kinetic theory of active particles. Phys. Life Rev. 2016, 16, 123-139. [CrossRef] [PubMed]

2. Burini, D.; De Lillo, S.; Gibelli, L. Learning dynamics towards modeling living systems: reply to comments on "Collective learning dynamics modeling based on the kinetic theory of active particles". Phys. Life Rev. 2016, 16, 158-162. [CrossRef] [PubMed]

3. Burini, D.; Gibelli, L.; Outada, N. A kinetic theory approach to the modeling of complex living systems. In Active Particles; Bellomo, N., Degond, P., Tadmor, E., Eds.; Birkhäuser: Basel, Switzerland, 2017; Volume 1, pp. 229-258.

4. Bellomo, N.; Bellouquid, A.; Gibelli, L.; Outada, N. A Quest towards a Mathematical Theory of Living Systems; Birkhäuser: Basel, Switzerland, 2017.

5. Hofbauer, J.; Sigmund, K. Evolutionary game dynamics. Bull. Am. Math. Soc. 2003, 40, 479-519. [CrossRef]

6. Nowak, M.A. Evolutionary Dynamics. Exploring the Equations of Life; Harvard University Press: Cambridge, MA, USA, 2006.

7. Bellomo, N.; Knopoff, D.; Soler, J. On the difficult interplay between life, "complexity", and mathematical sciences. Math. Mod. Meth. Appl. Sci. 2013, 23, 1861-1913. [CrossRef]

8. Bellomo, N.; Herrero, M.A.; Tosin, A. On the dynamics of social conflicts: Looking for the Black Swan. Kinet. Relat. Models 2013, 6, 459-479.

9. Dolfin, M.; Leonida, L.; Outada, N. Modelling human behaviour in economics and social science. Phys. Life Rev. 2017, 22, 1-21. [CrossRef] [PubMed]

10. Vygotsky, L. Interaction between learning and development. Read. Dev. Child. 1978, 23, 34-41.

11. Piaget, J. "Piaget's Theory" Piaget and His School; Springer: Berlin/Heidelberg, Germany, 1976; pp. 11-23.

12. Bandura, A. Human agency in social cognitive theory. Am. Psychol. 1989, 44, 1175-1184. [CrossRef]

13. Cole, M.; Engestrom, Y. A cultural-historical approach to distributed cognition. In Distributed Cognitions: Psychological and Educational Considerations; Salomon, G., Ed.; Cambridge University Press: New York, NY, USA, 1993; pp. 1-46.

14. Munsterberg, H. Psychology, General and Applied; D. Appleton: Boston, MA, USA, 1915.

15. Gardner, H. The Mind's New Science: A History of the Cognitive Revolution; Basic Books: New York, NY, USA, 1987. 
16. Salomon, G.; Perkins, D.N. Individual and social aspects of learning. Rev. Res. Educ. 1998, 23, 1-24. [CrossRef]

17. Armitage, D.; Marschke, M.; Plummer, R. Adaptive co-management and the paradox of learning. Glob. Environ. Chang. 2008, 18, 86-98. [CrossRef]

18. Reed, M.; Evely, A.C.; Cundill, G.; Fazey, I.; Glass, J.; Laing, A.; Newig, J.; Parrish, B.; Prell, C.; Raymond, C.; et al. What is social learning? Ecol. Soc. 2010, 15, r1. [CrossRef]

19. Borowski, I.; Le Bourhis, J.; Pahl-Wostl, C.; Barraqué, B. Spatial misfit in participatory river basin management: Effects on social learning, a comparative analysis of German and French case studies. Ecol. Soc. 2008, 13, 7. [CrossRef]

20. Kuper, M.; Dionnet, M.; Hammani, A.; Bekkar, Y.; Garin, P.; Bluemling, B. Supporting the shift from state water to community water: Lessons from a social learning approach to designing joint irrigation projects in Morocco. Ecol. Soc. 2009, 14, 19. [CrossRef]

21. Pahl-Wostl, C.; Sendzimir, J.; Jeffrey, P.; Aerts, J.; Berkamp, G.; Cross, K. Managing change toward adaptive water management through social learning. Ecol. Soc. 2007, 12, 30. [CrossRef]

22. Davidson-Hunt, I.; Berkes, F. Learning as you journey: Anishinaabe perception of social-ecological environments and adaptive learning. Conserv. Ecol. 2003, 8, 5. [CrossRef]

23. Fazey, J.A.; Martonl, F. Understanding the space of experiential variation. Act. Learn. High. Educ. 2002, 3, 234-250. [CrossRef]

24. Esteva, A.; Kuprel, B.; Novoa, R.A.; Ko, J.; Swetter, S.M.; Blau, H.M.; Thrun, S. Dermatologist-level classification of skin cancer with deep neural networks. Nature 2017, 542, 115-118. [CrossRef]

25. Garavan, T.N.; Carbery, R. Collective Learning; Encyclopedia of the Sciences of Learning; Springer: Boston, MA, USA, 2012; pp. 646-649.

26. Capello, R. Spatial transfer of knowledge in high technology milieux: Learning versus collective learning processes. Reg. Stud. 1999, 33, 353-365. [CrossRef]

27. Dosi, G. Technological paradigms and technological trajectories: A suggested interpretation of the determinants and directions of technical change. Res. Policy 1982, 11, 147-162. [CrossRef]

28. Nelson, R.R.; Winter, S.G. An Evolutionary Theory of Economic Change; Harvard University Press: Cambridge, MA, USA, 1982.

29. Castellano, C.; Fortunato, S.; Loreto, V. Statistical physics of social dynamics. Rev. Mod. Phys. 2009, 81, 591. [CrossRef]

30. Weidlich, W. The statistical description of polarization phenomena in society. Br. J. Math. Stat. Psychol. 1971, 24, 251-266. [CrossRef]

31. Galam, S.; Gefen, Y.; Shapir, Y. Sociophysics: A new approach of sociological collective behaviour. I. mean-behaviour description of a strike. J. Math. Sociol. 1982, 9, 1-13. [CrossRef]

32. Galam, S.; Moscovici, S. Towards a theory of collective phenomena: Consensus and attitude changes in groups. Eur. J. Soc. Psychol. 1991, 21, 49-74. [CrossRef]

33. Holley, R.A.; Liggett, T.M. Ergodic theorems for weakly interacting infinite systems and the voter model. Ann. Probab. 1975, 3, 643-663. [CrossRef]

34. Clifford, P.; Sudbury, A. A model for spatial conflict. Biometrika 1973, 60, 581-588. [CrossRef]

35. Carrillo, J.A.; Fornasier, M.; Rosado, J.; Toscani, G. Asymptotic flocking dynamics for the kinetic Cucker-Smale model. SIAM J. Math. Anal. 2010, 42, 218-236. [CrossRef]

36. Pareschi, L.; Toscani, G. Interacting Multiagent Systems: Kinetic Equations and Monte Carlo Methods; Oxford University Press: New York, NY, USA, 2013.

37. Toscani, G. Kinetic models of opinion formation. Commun. Math. Sci. 2006, 4, 481-496. [CrossRef]

38. Axelrod, R. The dissemination of culture: A model with local convergence and global polarization. J. Confl. Resolut. 1997, 41, 203-226. [CrossRef]

39. Czirók, A.; Barabási, A.L.; Vicsek, T. Collective motion of self-propelled particles: Kinetic phase transition in one dimension. Phys. Rev. Lett. 1999, 82, 209-212. [CrossRef]

40. Parrish, J.K.; Hamner, W.M. Animal Groups in Three Dimensions: How Species Aggregate; Cambridge University Press: Cambridge, UK, 1997.

41. Hankin, B.D.; Wright, R.A. Passenger flow in subways. J. Oper. Res. Soc. 1958, 9, 81-88. [CrossRef]

42. Henderson, L.F. The statistics of crowd fluids. Nature 1971, 229, 381-383. [CrossRef] [PubMed] 
43. Bellomo, N.; Bellouquid, A.; Knopoff, D. From the microscale to collective crowd dynamics. Multiscale Model. Simul. 2013, 11, 943-963. [CrossRef]

44. Bellomo, N.; Gibelli, L. Toward a mathematical theory of behavioral-social dynamics for pedestrian crowds. Math. Mod. Meth. Appl. Sci. 2015, 25, 2417-2437. [CrossRef]

45. Bellomo, N.; Gibelli, L.; Outada, N. On the interplay between behavioral dynamics and social interactions in human crowds. Kinet. Relat. Models 2019, 12, 397-409. [CrossRef]

46. Sigmund, K. The Calculus of Selfishness; Princeton University Series in Theoretical and Computational Biology; Princeton University Press: Princeton, NJ, USA, 2011.

47. Knopoff, D. On the modeling of migration phenomena on small networks. Math. Mod. Meth. Appl. Sci. 2013, 23, 541-563. [CrossRef]

48. Knopoff, D. On a mathematical theory of complex systems on networks with application to opinion formation. Math. Mod. Meth. Appl. Sci. 2014, 24, 405-426. [CrossRef]

49. Bonacich, P.; Lu, P. Introduction to Mathematical Sociology; Princeton University Press: Princeton, NJ, USA, 2012.

50. Dolfin, M.; Lachowicz, M. Modeling altruism and selfishness in welfare dynamics: The role of nonlinear interactions. Math. Mod. Meth. Appl. Sci. 2014, 24, 2361-2381. [CrossRef]

51. Dolfin, M.; Lachowicz, M. Modeling opinion dynamics: How the network enhances consensus. Netw. Heterog. Media 2015, 10, 421-441. [CrossRef]

52. Dolfin, M.; Knopoff, D.; Leonida, L.; Maimone Ansaldo Patti, D. Escaping the trap of 'blocking': A kinetic model linking economic development and political competition. Kinet. Relat. Models 2017, 10, 423-443. [CrossRef]

53. Bertotti, M.L.; Delitala, M. From discrete kinetic and stochastic game theory to modelling complex systems in applied sciences. Math. Mod. Meth. Appl. Sci. 2004, 14, 1061-1084. [CrossRef]

54. Bisi, M.; Spiga, G.; Toscani, G. Kinetic models of conservative economies with wealth redistribution. Commun. Math. Sci. 2009, 7, 901-916. [CrossRef]

55. Aletti, G.; Naldi, G.; Toscani, G. First-order continuous models of opinion formation. SIAM J. Appl. Math. 2007, 67, 837-853. [CrossRef]

56. Caponigro, M.; Lai, A.C.; Piccoli, B. A nonlinear model of opinion formation on the sphere. Discret. Cont. Dyn. Syst. Ser. A 2015, 35, 4241-4268.

57. Aimone Marsan, G.; Bellomo, N.; Gibelli, L. Stochastic evolutionary differential games toward a systems theory of behavioral social dynamics. Math. Mod. Meth. Appl. Sci. 2016, 26, 1051-1093. [CrossRef]

58. Bellomo, N.; Bellouquid, A. On multiscale models of pedestrian crowds from mesoscopic to macroscopic. Commun. Math. Sci. 2015, 13, 1649-1664. [CrossRef]

59. Bellomo, N.; Bellouquid, A.; Nieto, J.; Soler, J. On the multiscale modeling of vehicular traffic: From kinetic to hydrodynamics. Discret. Cont. Dyn. Syst. B 2014, 19, 1869-1888. [CrossRef]

60. Bellomo, N.; Ha, S.Y. A quest toward a mathematical theory of the dynamics of swarms. Math. Mod. Meth. Appl. Sci. 2017, 27, 745-770. [CrossRef]

61. Di Francesco, M.; Fagioli, S. A nonlocal swarm model for predators-prey interactions. Math. Mod. Meth. Appl. Sci. 2016, 26, 319-355. [CrossRef]

62. Bellouquid, A.; De Angelis, E.; Knopoff, D. From the modeling of the immune hallmarks of cancer to a black swan in biology. Math. Mod. Meth. Appl. Sci. 2013, 22, 949-978. [CrossRef]

63. Hanahan, D.; Weinberg, R.A. Hallmarks of cancer: The next generation. Cell 2011, 144, 646-674. [CrossRef]

64. Weinberg, R.A. The Biology of Cancer; Garland Sciences-Taylor and Francis: New York, NY, USA, 2007.

65. Cooper, E.L. Evolution of immune system from self/not self to danger to artificial immune system. Phys. Life Rev. 2010, 7, 55-78. [CrossRef] [PubMed]

66. Bellouquid, A.; Delitala, M. Mathematical Modeling of Complex Biological Systems; Birkhäuser: Basel, Switzerland, 2006.

67. De Angelis, E. On the mathematical theory of post-Darwinian mutations, selection, and evolution. Math. Mod. Meth. Appl. Sci. 2014, 24, 2723-2742. [CrossRef]

68. Cavallo, F.; De Giovanni, C.; Nanni, P.; Forni, G.; Lollini, P.L. 2011: The immune hallmarks of cancer. Cancer Immunol. 2011, 60, 319-326. [CrossRef] [PubMed]

69. Aristov, V.V. Direct Methods for Solving the Boltzmann Equation and Study of Nonequilibrium Flows; Springer: New York, NY, USA, 2001 
70. Bird, G.A. Molecular Gas Dynamics and the Direct Simulation of Gas Flows; Oxford University Press: Oxford, UK, 1994.

71. Albi, G.; Bellomo, N.; Fermo, L.; Ha, S.-Y.; Kim, J.; Pareschi, L.; Poyato, D.; Soler, J. Traffic, crowds, and swarms. From kinetic theory and multiscale methods to applications and research perspectives. Math. Mod. Meth. Appl. Sci. 2019, 29. [CrossRef]

72. Barbante, P.; Frezzotti, A.; Gibelli, L. A kinetic theory description of liquid menisci at the microscale. Kinet. Relat. Mod. 2015, 8, 235-254.

73. Burini, D.; Chouhad, N. Hilbert method toward a multiscale analysis from kinetic to macroscopic models for active particles. Math. Mod. Meth. Appl. Sci. 2017, 27, 1327-1353. [CrossRef]

74. Burini, D.; Chouhad, N. A multiscale view of nonlinear diffusion in biology: From cells to tissues. Math. Mod. Meth. Appl. Sci. 2019, 29, 791-823. [CrossRef]

(C) 2019 by the authors. Licensee MDPI, Basel, Switzerland. This article is an open access article distributed under the terms and conditions of the Creative Commons Attribution (CC BY) license (http:/ / creativecommons.org/licenses/by/4.0/). 
Article

\title{
Diffusive and Anti-Diffusive Behavior for Kinetic Models of Opinion Dynamics
}

\author{
Mirosław Lachowicz ${ }^{1, *,+}$, Henryk Leszczyński ${ }^{2,+}$ and Elżbieta Puźniakowska-Gałuch ${ }^{2, \dagger}$ \\ 1 Institute of Applied Mathematics and Mechanics, Faculty of Mathematics, Informatics and Mechanics, \\ University of Warsaw, ul. Banacha 2, 02-097 Warsaw, Poland \\ 2 Institute of Mathematics, University of Gdańsk, ul. Wita Stwosza 57, 80-308 Gdańsk, Poland \\ * Correspondence: M.Lachowicz@mimuw.edu.pl \\ + These authors contributed equally to this work.
}

Received: 12 July 2019; Accepted: 31 July 2019; Published: 8 August 2019

check for updates

\begin{abstract}
In the present paper, we study a class of nonlinear integro-differential equations of a kinetic type describing the dynamics of opinion for two types of societies: conformist $(\sigma=1)$ and anti-conformist $(\sigma=-1)$. The essential role is played by the symmetric nature of interactions. The class may be related to the mesoscopic scale of description. This means that we are going to statistically describe an individual state of an agent of the system. We show that the corresponding equations result at the macroscopic scale in two different pictures: anti-diffusive $(\sigma=1)$ and diffusive $(\sigma=-1)$. We provide a rigorous result on the convergence. The result captures the macroscopic behavior resulting from the mesoscopic one. In numerical examples, we observe both unipolar and bipolar behavior known in political sciences.
\end{abstract}

Keywords: opinion dynamics; symmetric interactions; kinetic equations; integro-differential equations; conformist society; individualistic society

\section{Introduction}

In Ref. [1] a general model of swarming behavior of an individual population was proposed and studied. The main aim in that paper was to study the macroscopic (so-called "hydrodynamic") limit. The mathematical structure proposed therein seems very rich and interesting from the mathematical point of view-see the analysis of its simplification in Refs. [2-4].

In the present paper, we study the time evolution of the probability density $f$. The function $f=f(t, u)$ is the distribution of an internal, microscopic state $u \in \mathbb{R}^{d}$ at time $t \geq 0$ of a (statistical or test) agent. Such a description has then a mesoscopic nature. An arbitrary vector $u \in \mathbb{R}^{d}$ can be related to a biological state, activity, opinion (e.g., political opinion), a social state of a test agent, etc.-cf. $[1,5-7]$ and references therein, $d=1,2, \ldots$. The model has therefore a wide range of possible applications in various applied sciences, such as biology, medicine, social or political sciences.

Because some results of the present paper may have a particular importance in the description in social sciences, we will refer the parameter $u$ to as the opinion of (test) agent.

The time evolution is defined by the general nonlinear integro-differential Boltzmann-like equation, see Ref. [1] and references therein,

$$
\partial_{t} f(t, u)=Q[f](t, u), \quad u \in \mathbb{R}^{d}, \quad t \geq 0,
$$

where

$$
Q[f](t, u)=\int_{\mathbb{R}^{d}}(T[f(t, .)](v, u) f(t, v)-f(t, u) T[f(t, .)](u, v)) \mathrm{d} v .
$$


The nonlinear operator $Q$ describes interactions between agents causing a change of opinion. The turning rate $T[f](u, v)$ measures the rate for an agent with opinion $u$ to change it into $v$. A simpler equation, with two possible states only, was studied in Ref. [8]—see also Ref. [6]. It is a typical kinetic (microscopic or more precisely, mesoscopic) way of describing large (social) populations. Instead of dealing with the states of all separated agents involved in the population evolution, we deal with the statistical state of one (test) agent. This state is described by the probability density $f$ that is a solution of Equation (1). The main aim is to describe general dispersion-concentration phenomena resulting from individual opinions of agents. The dispersion results in the presence of various opinions in a society, whereas the concentration represents a kind of uniformity of opinions. The main advantage of the class of models given by Equation (1) is that it can capture many scenarios that are known in social sciences.

There is an increasing interest in a kinetic approach in a similar context. Ref. [9] is dedicated to the modeling and simulation of swarms, where interactions at the microscopic scale are modeled by stochastic games. Ref. [10] describes the state of the art of swarming at the individual-based level and the macroscopic level. Ref. [5] models ensembles of social agents as behavioral, evolutionary, complex systems referring to the complexity features of living systems. Individual-based approaches based on stochastic processes are proposed in Refs. [7,11,12]. Ref. [13] studies the local stability of Dirac masses for a kinetic model of alignment. See also references therein.

The modeling process leads to a proper choice of the turning rate. In the present paper, we are going to study two different and somehow opposite choices.

Case 1. (A conformist society). Let

$$
T[f(t, .)](u, v)=\beta(u, v) f^{\gamma}(t, v), \quad u, v \in \mathbb{R}^{d},
$$

where $\gamma>1$ is a given number.

Throughout the paper we assume that $\beta$ is a symmetric function of its arguments, i.e.,

$$
\beta(u, v)=\beta(v, u) \quad \text { for all } u, v \in \mathbb{R}^{d} .
$$

This assumption, which may be related to a symmetric nature of interactions between agents, is essential for the mathematical theory presented here. In fact relaxing of the assumption leads to a completely different behavior and different macroscopic limits. In such a case one may expect first order terms that dominate in the macroscopic picture causing a drift towards some states.

The rate of transition from opinion $u$ to opinion $v$ is proportional to $\gamma$-power of the actual probability of having opinion $v$. The higher the probability, the larger the chance of a change. Therefore it is natural to refer to such a society as a conformist society (or a collectivistic society). The interaction kernel $\beta$ corresponds to the tendency of agents to change opinion. In particular, it may restrict the interactions to close opinions only—see Ref. [4]. The (sensitivity) parameter $\gamma$ describes the level of sensitivity of interactions. The greater $\gamma$, the more sensitive interactions.

The models defined by Case 1 was proposed in Ref. [1], and then studied in various directions in Refs. [2-4]. Ref. [1] proposed results of global existence in the space homogeneous case for $0<\gamma<1$, whereas $\gamma>1$ was considered in Ref. [2-4]. Assuming that $\gamma=1$ yields a trivial model, thus it is excluded. Alternatively, we may propose:

Case 2. (A moderately anti-conformist society). Let

$$
T[f(t, .)](u, v)=\beta(u, v) f(t, v) f^{\gamma-1}(t, u), \quad u, v \in \mathbb{R}^{d},
$$

where $\gamma>1$ is a given number. 
The rate of transition from opinion $u$ to opinion $v$ is proportional to the product of $\gamma-1$-power of the actual probability of having opinion $u$ and the actual probability of having opinion $v$. The higher the product, the larger the chance of a change. Therefore, we may refer to such a society as a moderately anti-conformist society (or a individualistic society). As before, the interaction kernel $\beta$ corresponds to the tendency of agents to change opinion.

One may note that the following choice of $T[f(t,)$.$] leads to the same kinetic equation,$ again justifying its name (the moderately anti-conformist society) (or individualistic society).

Case 3. Let

$$
T[f(t, .)](u, v)=\beta(u, v) f(t, v)\left(M^{\gamma-1}(t)-f^{\gamma-1}(t, v)\right), \quad u, v \in \mathbb{R}^{d}
$$

where $\gamma>1$ is a given number and $M(t)=\operatorname{ess} \sup _{u \in \mathbb{R}^{d}} f(t, u)$.

Both Cases 1 and 2 (or 3) can be put together as the following equation

$$
\begin{aligned}
& \partial_{t} f(t, u)=Q[f](t, u)= \\
& =\sigma \int_{\mathbb{R}^{d}}\left(\beta(v, u) f(t, v) f^{\gamma}(t, u)-\beta(v, u) f(t, u) f^{\gamma}(t, v)\right) \mathrm{d} v, \\
& u \in \mathbb{R}^{d}, \quad t \geq 0
\end{aligned}
$$

where $\sigma= \pm 1$. Condition $\sigma=1$ corresponds to Case 1 , whereas $\sigma=-1$ to Case 2 (or Case 3).

The main Equation (3) reads

$$
\partial_{t} f(t, u)=\sigma\left((\beta * f)(t, u) f^{\gamma}(t, u)-f(t, u)\left(\beta * f^{\gamma}\right)(t, u)\right)
$$

$u \in \mathbb{R}^{d}, t \geq 0$, whereby $(\beta * f)$ we denote the convolution-like product, i.e.,

$$
(\beta * f)(t, u)=\int_{\mathbb{R}^{d}} \beta(u, v) f(t, v) \mathrm{d} v .
$$

The $L^{p}$-norm is denoted by $\|.\|_{p}$. We may state the following existence-uniqueness result for solutions to Equation (4).

Proposition 1. Let $\gamma>1$. If $f_{0}$ is a probability density such that $f_{0} \in L^{\infty}\left(\mathbb{R}^{d}\right)$, then there exists $T>0$ such that the solution $f=f(t)$ to (4) exists and is unique in $L^{\infty}\left(\mathbb{R}^{d}\right) \cap L^{1}\left(\mathbb{R}^{d}\right)$ on the interval $[0, T)$. The solution preserves the positivity and $L^{1}$-norm (i.e., it is a probability density) on $[0, T)$. Moreover,

1. If $\sigma=-1$ the solution is global and possesses all finite $L^{p}$-norms, $p>1$, and the functions $t \mapsto\|f(t)\|_{p}$ are decreasing;

2. If $\sigma=1$ the solution, depending on initial data, is either global $(T=\infty)$ or local $(T<\infty)$, it possesses all finite $L^{p}$-norms on $[0, T), p>1$, and the functions $t \mapsto\|f(t)\|_{p}$ are increasing for $t \in[0, T)$.

Proof. The proof follows from Ref. [1]. First we observe that the operator on the right-hand side of Equation (4) is locally Lipschitz-continuous in the $L^{\infty}\left(\mathbb{R}^{d}\right) \cap L^{1}\left(\mathbb{R}^{d}\right)$-setting. Therefore, the existence in this setting on some interval $[0, T)$ follows. It is easy to see that the solution $f=f(t)$ preserves positivity and $L^{1}\left(\mathbb{R}^{d}\right)$-norm of the initial datum, $t \in[0, T)$. 
Let $\lambda(x)=x^{p}, p>1$. Then the solution $f=f(t)$ a priori satisfies

$$
\begin{aligned}
& \frac{\mathrm{d}}{\mathrm{d} t} \int_{\mathbb{R}^{d}} \lambda(f(t, u)) \mathrm{d} u=\frac{\sigma}{2} \int_{\mathbb{R}^{d}} \int_{\mathbb{R}^{d}} \beta(u, v) f(t, u) f(t, v) \times \\
& \left(f^{\gamma-1}(t, u)-f^{\gamma-1}(t, v)\right)\left(\lambda^{\prime}(f(t, u))-\lambda^{\prime}(f(t, v))\right) \mathrm{d} v \mathrm{~d} u
\end{aligned}
$$

Therefore, the conclusion follows.

Moreover, we need the smoothness of the solutions. Let $W^{m, p}\left(\mathbb{R}^{d}\right)$ and $C_{B}^{m}\left(\mathbb{R}^{d}\right)$ be the Banach spaces-the classical Sobolev space (a subspace of $L^{p}\left(\mathbb{R}^{d}\right)$ ) and the space of $m$-differentiable functions, respectively, with the usual norms denoted by $\|\cdot\|_{p}^{(m)}$ and $\|\cdot\|_{[B]}^{(m)}$, respectively.

Let $X^{(m)}=W^{m, 1}\left(\mathbb{R}^{d}\right) \cap C_{B}^{m}\left(\mathbb{R}^{d}\right), m=0,1,2, \ldots$, and $\|\cdot\|^{(m)}$ be defined

$$
\|\cdot\|^{(m)}=\|\cdot\|_{1}^{(m)}+\|\cdot\|_{[B]}^{(m)}, \quad m=0,1,2, \ldots .
$$

In particular, for $m=0$, we write $X=X^{(0)}=L^{1}\left(\mathbb{R}^{d}\right) \cap L^{\infty}\left(\mathbb{R}^{d}\right)$ and $\|\cdot\|=\|\cdot\|^{(0)}$.

Proposition 2. Let the assumption of Proposition 1 be satisfied and additionally $f_{0} \in X^{(m)}$ and

$$
(\beta * g) \in X^{(m)} \quad \text { for each } g \in X^{(m)},
$$

for some $m=1,2,3, \ldots$ Then the solution $f=f(t)$ (given by Proposition 1 ) satisfies $f(t,.) \in X^{(m)}$ for all $t \in[0, T)$.

Proof. The proof follows again by the standard application of Lipschitz-continuity in $X^{(m)}$.

\section{Formal Diffusive and Anti-Diffusive Limits}

In the present section we study (only in a formal way) the macroscopic limits of Equation (4) with both $\sigma=1$ and $\sigma=-1$. As we will see, the case $\sigma=-1$ corresponds to the diffusive macroscopic behavior, whereas $\sigma=1$-to the anti-diffusive behavior.

Suppose that the support of $\beta$ is concentrated close to the diagonal $\left\{(u, v) \in \mathbb{R}^{2 d}: u=v\right\}$, i.e.,

$$
\beta(u, v)=\frac{1}{\varepsilon^{d+2} \omega_{d}} \chi(|u-v| \leq \varepsilon),
$$

where $\omega_{d}$ is the normalizing coefficient, i.e., $d$-dimensional volume of the unit ball (centered at 0 ).

We are going to consider $\varepsilon \rightarrow 0$, because we are interested in the limit as the "support tends to the diagonal" and the non-zero values of $\beta$ tend to infinity. We may note that $\beta$ defined in Equation (6) satisfies Equations (5). The essential role here is again played by the symmetric nature of interactions. Without this nature, the limit could be completely different.

Let the solution of Equation (4), for fixed $\varepsilon>0$, be denoted by $f^{(\varepsilon)}=f^{(\varepsilon)}(t)$ and be in $X^{(3)}$. If $f^{(\varepsilon)}$ tends to $f$ in $X^{(3)}$, then $f$ is a solution to the following partial differential equation

$$
\partial_{t} f=\sigma(1-\gamma) \frac{c_{d}}{2 \omega_{d}} \nabla \cdot\left(f^{\gamma} \nabla f\right)
$$

with the positive constant $c_{d}$

$$
c_{d}=\int_{\mathbb{B}(1)}\left(v_{i}\right)^{2} \mathrm{~d} v,
$$

where $\mathbb{B}(1)$ is the unit ball. Taking $\gamma>1$ as well as $\sigma=-1$, Equation (7) is the classical porous medium equation-see Refs. [14-16] and references therein. On the other hand, taking $\gamma>1$ and $\sigma=1$ we obtain a nonlinear anti-diffusion equation. A linear anti-diffusion equation was also formally 
obtained as in a hydrodynamic limit in Ref. [8]—see (4.19) and (4.20) therein. Considering regular solutions (i.e., $C^{2}$ with respect to $u$ ), Equation (7) is equivalent to

$$
\partial_{t} f=\frac{\sigma c_{d}}{2 \omega_{d}}\left(f^{\gamma} \Delta f-f \Delta\left(f^{\gamma}\right)\right),
$$

where $\Delta$ is the Laplace operator. We generalize the above argument to Equation (4) with $\beta$ given by

$$
\beta(u, v)=\frac{1}{\varepsilon^{d+2}} \bar{\beta}\left(\frac{u-v}{\varepsilon}\right) .
$$

We do not assume that $\bar{\beta}$ is a probability density but we refer to the notion of generalized moments as well as the generalized covariance matrix in a standard way.

\section{Assumption 1. We assume}

1. The function $\bar{\beta}: \mathbb{R}^{d} \rightarrow \mathbb{R}$ is bounded, non-negative and symmetric, i.e., $\bar{\beta}(v)=\bar{\beta}(-v)$;

2. The first, second, third, and fourth generalized moments are bounded, i.e.,

$$
\int_{\mathbb{R}^{d}}|v|^{p} \bar{\beta}(v) \mathrm{d} v<+\infty \quad \text { for } p=1,2,3,4 ;
$$

3. The matrix

$$
\Sigma_{\bar{\beta}}=\left[\int_{\mathbb{R}^{d}} v_{i} v_{j} \bar{\beta}(v) \mathrm{d} v\right],
$$

the generalized covariance matrix of $\bar{\beta}$, is positive definite.

If $\bar{\beta}$ is a probability density, then the generalized moments and the generalized covariance of $\bar{\beta}$ become just moments and covariance of $\bar{\beta}$. Assumption 1 is satisfied for $\beta$ in Equation (6) if $\bar{\beta}$ is given by

$$
\bar{\beta}(v)=\frac{1}{\omega_{d}} \chi(|u-v| \leq 1) .
$$

With this $\bar{\beta}$ we put

$$
\beta(u, v)=\frac{1}{\varepsilon^{d+2}} \bar{\beta}\left(\frac{u-v}{\varepsilon}\right),
$$

and easily obtain Equation (7), which is formally equivalent to Equation (9). We intend to obtain the following generalization of Equation (9)

$$
\partial_{t} f(t, u)=\frac{\sigma}{2}\left\{f^{\gamma}(t, u) \Sigma_{\bar{\beta}} \cdot D^{2} f(t, u)-f(t, u) \Sigma_{\bar{\beta}} \cdot D^{2} f^{\gamma}(t, u)\right\} .
$$

The symbol "." stands for the inner scalar product of two $d \times d$-matrices and $D^{2} f$ is the Hessian matrix (it consists of second order derivatives in $u_{i}, u_{j}$ ). To obtain Equation (11) we use the Taylor expansions

$$
\begin{aligned}
f^{(\varepsilon)}(t, v)=f^{(\varepsilon)}(t, u)+ & (v-u) \cdot \nabla f^{(\varepsilon)}(t, u)+\frac{1}{2}(v-u) \cdot D^{2} f^{(\varepsilon)}(t, u)(v-u)+\mathcal{O}\left(|v-u|^{3}\right), \\
\left(f^{(\varepsilon)}\right)^{\gamma}(t, v) & =\left(f^{(\varepsilon)}\right)^{\gamma}(t, u)+(v-u) \cdot \nabla\left(f^{(\varepsilon)}\right)^{\gamma}(t, u) \\
& +\frac{1}{2}(v-u) \cdot D^{2}\left(f^{(\varepsilon)}\right)^{\gamma}(t, u)(v-u)+\mathcal{O}\left(|v-u|^{3}\right) .
\end{aligned}
$$


Substituting these expressions to the right-hand side of Equation (4) and taking into account Equation (10) we obtain

$$
\begin{aligned}
& \sigma \int_{\mathbb{R}^{d}}\left(\beta(u, v) f^{(\varepsilon)}(t, v)\left(f^{(\varepsilon)}\right)^{\gamma}(t, u)-\beta(u, v) f^{(\varepsilon)}(t, u)\left(f^{(\varepsilon)}\right)^{\gamma}(t, v)\right) \mathrm{d} v \\
& =\frac{\sigma}{\varepsilon^{d+2}} \int_{\mathbb{R}^{d}} \bar{\beta}\left(\frac{u-v}{\varepsilon}\right)\left(f^{(\varepsilon)}\right)^{\gamma}(t, u)\left(f^{(\varepsilon)}(t, u)+\nabla f^{(\varepsilon)}(t, u) \cdot(v-u)\right. \\
& \left.\quad+\frac{1}{2}(v-u) \cdot D^{2} f^{(\varepsilon)}(t, u)(v-u)+\mathcal{O}\left(|v-u|^{3}\right)\right) \mathrm{d} v \\
& \quad-\frac{\sigma}{\varepsilon^{d+2}} \int_{\mathbb{R}^{d}} \bar{\beta}\left(\frac{u-v}{\varepsilon}\right) f^{(\varepsilon)}(t, u)\left(\left(f^{(\varepsilon)}\right)^{\gamma}(t, u)+\nabla\left(f^{(\varepsilon)}\right)^{\gamma}(t, u) \cdot(v-u)\right. \\
& \left.\quad+\frac{1}{2}(v-u) \cdot D^{2}\left(f^{(\varepsilon)}\right)^{\gamma}(t, u)(v-u)+\mathcal{O}\left(|v-u|^{3}\right)\right) \mathrm{d} v
\end{aligned}
$$

From the symmetry of $\bar{\beta}$, the first order terms with respect to $v-u$ vanish. The second order terms yield

$$
\frac{\sigma}{2}\left\{\left(f^{(\varepsilon)}\right)^{\gamma}(t, u) \Sigma_{\bar{\beta}} \cdot D^{2}\left(f^{(\varepsilon)}\right)(t, u)-f^{(\varepsilon)}(t, u) \Sigma_{\bar{\beta}} \cdot D^{2}\left(f^{(\varepsilon)}\right)^{\gamma}(t, u)\right\} .
$$

In the limit $\varepsilon \rightarrow 0$ we formally obtain the diffusion or anti-diffusion equation Equation (11).

In the particular case of Equation (6), all mixed (generalized) second moments of $\bar{\beta}(v)=\frac{1}{\omega_{d}} \chi(|u-v| \leq 1)$ are equal to zero. In that case the pure second (generalized) moments, using Equation (8), are as follows

$$
\begin{aligned}
\sigma \int_{\mathbb{R}^{d}} & \left(\beta(v, u) f^{(\varepsilon)}(t, v)\left(f^{(\varepsilon)}\right)^{\gamma}(t, u)-\beta(v, u) f^{(\varepsilon)}(t, u)\left(f^{(\varepsilon)}\right)^{\gamma}(t, v)\right) \mathrm{d} v \\
\quad= & \frac{\sigma \mathcal{c}_{d}}{2 \omega_{d}}\left(\left(f^{(\varepsilon)}\right)^{\gamma}(t, u) \nabla^{2} f^{(\varepsilon)}(t, u)-f^{(\varepsilon)}(t, u) \nabla^{2}\left(\left(f^{(\varepsilon)}\right)^{\gamma}(t, u)\right)\right)+\mathcal{O}(\varepsilon) .
\end{aligned}
$$

In this case, in the limit $\varepsilon \rightarrow 0$, Equation (4) formally results in the diffusion/anti-diffusion equation Equation (9). Also, formally, Equation (9) turns to be a porous-media equation when $\sigma=-1$. The existence, uniqueness and regularity criteria are formulated in [15]. There is no such result for anti-diffusion equations, in particular for Equation (9) with $\sigma=1$.

\section{Convergence Result}

In this section, we are going to prove the rigorous convergence result - a diffusive limit for $\sigma=-1$. It is somehow in the spirit of the diffusive limit for a class of bilinear equations studied in Ref. [17]. We assume stronger regularity of the initial datum $f_{0}$ and obtain the convergence of order $\varepsilon^{2}$. Due to the symmetry of $\beta$ one can enhance the convergence rate from $\varepsilon^{1}$ to $\varepsilon^{2}$.

Concerning Equation (4) with $\sigma=-1$ the global existence and uniqueness follows from Proposition 1, for its diffusive limit (7)—from [15] (by the porous-media theory), whereas the case $\sigma=1$ is almost completely unknown.

We transform Equation (11) to a porous-media equation, for which one can state an existence result in the diffusive case $\sigma=-1$. Since the matrix $\Sigma_{\bar{\beta}}$ is symmetric and positive-definite, there is a $d \times d$-matrix $B$ such that $\Sigma_{\bar{\beta}}=B^{2}$. We can now define a new function $F(t, u)=f(t, B u)$ and establish the relations between the derivatives

$$
\nabla F(t, u)=(B \nabla f)(t, B u) \quad \Delta F(t, u)=\left(B^{2} \cdot D^{2} f\right)(t, B u)=\left(\Sigma_{\bar{\beta}} \cdot D^{2} f\right)(t, B u) .
$$


Herewith Equation (11) transforms to

$$
\partial_{t} F=-\frac{1}{2} F^{\gamma} \Delta F+\frac{1}{2} F \Delta F^{\gamma}
$$

The initial condition is given by $F_{0}(u)=f_{0}(B u)$. It follows

$$
\nabla \cdot\left(F^{\gamma} \nabla F\right)(t, u)=\nabla \cdot\left(F^{\gamma}(t, u)(B \nabla f)(t, B u)\right)=\frac{1}{\gamma+1} B^{2} \cdot D^{2}\left(f^{\gamma+1}\right)(t, B u) .
$$

In this way we arrive at an equation which is formally equivalent to Equation (11) and reduces to a porous-media equation

$$
\partial_{t} f=\frac{\gamma-1}{2(\gamma+1)} \Sigma_{\bar{\beta}} \cdot D^{2}\left(f^{\gamma+1}\right) .
$$

The respective function $F(t, u)=f(t, B u)$ satisfies the porous-media equation

$$
\partial_{t} F=\frac{\gamma-1}{2(\gamma+1)} \Delta\left(F^{\gamma+1}\right)
$$

We are ready to formulate an existence result.

Proposition 3. Let $\gamma>1, \sigma=-1$ and $f_{0}$ be a probability density on $\mathbb{R}^{d}$ such that $f_{0} \in X^{(p)}$. Let moreover $\bar{\beta} \in X$ satisfy Assumption 1. Then Equation (17) with the initial condition $f(0)=f_{0}$ has a unique solution $f=f(t)$ in $X^{(p)}$, on some interval $[0, T]$.

Proof. By the substitution $F(t, u)=f(t, B u)$ Equations (17) reduces to (18), in other words

$$
\partial_{t} F=\frac{\gamma-1}{2} \nabla \cdot\left(F^{\gamma} \nabla F\right)
$$

with the initial condition $F_{0}(u)=f_{0}(B u)$. The assertion follows from the porous-media existence theory.

Theorem 1. Let $\gamma>1, \sigma=-1$ as well as $f_{0}$ be a probability density on $\mathbb{R}^{d}$ such that $f_{0} \in X^{(4)}$. Let moreover $\bar{\beta} \in X$ be a non-negative function satisfying Assumption 1 and let $f^{(\varepsilon)}=f^{(\varepsilon)}(t)$ defined on $[0, T] \times \mathbb{R}^{d}$ be a solution of Equation (4) with the initial datum $f_{0}$ and with

$$
\beta(u, v)=\frac{1}{\varepsilon^{d+2}} \bar{\beta}\left(\frac{u-v}{\varepsilon}\right) .
$$

If $f=f(t)$ in $X$ is a solution to Equation (17) with the same initial datum $f_{0}$, then

$$
\sup _{[0, T]}\left\|f^{(\varepsilon)}-f\right\| \leq C \varepsilon^{2}
$$

with some constant $C>0$.

Proof. Let $f^{(\varepsilon)} \in X^{(4)}$ be the solution to Equation (4) with $\beta$ defined by (10). The idea of the proof is as follows:

- the kinetic equation is transformed into an equation formally equivalent to a porous-media equation with a small perturbation;

- $\quad$ the perturbed porous-media equation is reduced to its standard form by the change of variables $u \rightarrow B u$;

- $\quad$ the comparison principle for porous-media equations is applied. 
We fix $(t, u) \in[0, T] \times \mathbb{R}^{d}$. Using the Taylor expansions to Equation (4) as in Section 2, we obtain the right-hand side given by Equation (12). From the symmetry of $\bar{\beta}$, the first and third order terms with respect to $v-u$ vanish. The second order terms yield

$$
\begin{aligned}
I_{\varepsilon}\left(f^{(\varepsilon)}\right)(t, u) & =\frac{-1}{2}\left\{\left(f^{(\varepsilon)}\right)^{\gamma}(t, u) \Sigma_{\bar{\beta}} \cdot D^{2}\left(f^{(\varepsilon)}\right)(t, u)-f^{(\varepsilon)}(t, u) \Sigma_{\bar{\beta}} \cdot D^{2}\left(f^{(\varepsilon)}\right)^{\gamma}(t, u)\right\} \\
& =\frac{\gamma-1}{2(\gamma+1)} \Sigma_{\bar{\beta}} \cdot\left(D^{2} f^{\gamma+1}\right) .
\end{aligned}
$$

Hence Equation (12) reads

$$
\begin{aligned}
& \int_{\mathbb{R}^{d}} \frac{1}{\varepsilon^{d+2}} \bar{\beta}\left(\frac{u-v}{\varepsilon}\right)\left(f^{(\varepsilon)}(t, v)\left(f^{(\varepsilon)}\right)^{\gamma}(t, u)-f^{(\varepsilon)}(t, u)\left(f^{(\varepsilon)}\right)^{\gamma}(t, v)\right) \mathrm{d} v \\
& \quad=I_{\varepsilon}\left(f^{(\varepsilon)}\right)(t, u)+R_{\varepsilon}\left(f^{(\varepsilon)}\right)(t, u)
\end{aligned}
$$

where

$$
\begin{aligned}
R_{\varepsilon}\left(f^{(\varepsilon)}\right)(t, u) & =\int_{\mathbb{R}^{d}} \frac{1}{\varepsilon^{d+2}} \bar{\beta}\left(\frac{u-v}{\varepsilon}\right)\left(f^{(\varepsilon)}\right)^{\gamma}(t, u) \int_{0}^{1} \frac{(1-\theta)^{3}}{6} D^{4} f^{(\varepsilon)}(t, u+\theta(v-u))(v-u)^{4} \mathrm{~d} \theta \mathrm{d} v \\
& +\int_{\mathbb{R}^{d}} \frac{1}{\varepsilon^{d+2}} \bar{\beta}\left(\frac{u-v}{\varepsilon}\right) f^{(\varepsilon)}(t, u) \int_{0}^{1} \frac{(1-\theta)^{3}}{6} D^{4}\left(f^{(\varepsilon)}\right)^{\gamma}(t, u+\theta(v-u))(v-u)^{4} \mathrm{~d} \theta \mathrm{d} v,
\end{aligned}
$$

where $D^{4}\left(f^{(\varepsilon)}\right)^{p}$ consists of all fourth-order partial derivatives $\partial_{u_{i} u_{j} u_{k} u_{l}}^{4}\left(f^{(\varepsilon)}\right)^{p}$ and $D^{4}\left(f^{(\varepsilon)}\right)^{p}(t, u+$ $\theta(v-u))(v-u)^{4}$ is the 4-linear form $D^{4}\left(f^{(\varepsilon)}\right)^{p}(t, u+\theta(v-u))$ acting on four copies of the vector $v-u$. The remainder $R_{\varepsilon}\left(f^{(\varepsilon)}\right)$ can be estimated by

$$
\int_{\mathbb{R}^{d}} \frac{1}{\varepsilon^{d+2}} \bar{\beta}\left(\frac{u-v}{\varepsilon}\right) f^{(\varepsilon)}(t, u)|v-u|^{4} \int_{0}^{1} \frac{(1-\theta)^{3}}{6}\left(\left\|f^{(\varepsilon)}\right\|_{L^{\infty}}^{\gamma-1}\left\|D^{4} f^{(\varepsilon)}\right\|_{L^{\infty}}+\left\|D^{4}\left(f^{(\varepsilon)}\right)^{\gamma}\right\|_{L^{\infty}}\right) \mathrm{d} \theta \mathrm{d} v,
$$

and the $X$-norm can be estimated by $C \varepsilon^{2}$ where $C$ is a constant depending on the fourth moments of $\bar{\beta}$ and the $L^{\infty}$-norm of $D^{4} f^{(\varepsilon)}$.

Finally, we attempt to a comparison of $f^{(\varepsilon)}$ with $f$. The above consideration leads to a perturbed porous-media equation for $f^{(\varepsilon)}$

$$
\partial_{t} f^{(\varepsilon)}(t, u)=\frac{\gamma-1}{2(\gamma+1)} \Sigma_{\bar{\beta}} \cdot D^{2}\left(f^{(\varepsilon)}\right)^{\gamma+1}+R_{\varepsilon}\left(f^{(\varepsilon)}\right)(t, u) .
$$

Alike the transformation $f \rightarrow F$, one can define $F^{(\varepsilon)}(t, u):=f^{(\varepsilon)}(t, B u)$, which satisfies

$$
\partial_{t} F^{(\varepsilon)}(t, u)=\frac{\gamma-1}{2(\gamma+1)} \Delta\left(f^{(\varepsilon)}\right)^{\gamma+1}+R_{\varepsilon}\left(f^{(\varepsilon)}\right)(t, B u)
$$

with the initial condition $F^{(\varepsilon)}(0, u)=F_{0}(u)=f_{0}(B u)$. Since $F$ satisfies the porous-media Equation (18) with the same initial datum, one can apply classical comparison theorems for porous media, see $[18,19]$. This leads to the estimate

$$
\sup _{[0, T]}\left\|F^{(\varepsilon)}-F\right\| \leq C \varepsilon^{2} .
$$

Since the matrix $B$ is non-singular, we obtain a similar estimate for $f^{(\varepsilon)}-f$. This completes the proof. 
If mixed moments (or mixed generalized moments) of $\bar{\beta}$ vanish, the covariance matrix $\Sigma_{\bar{\beta}}$ is a diagonal matrix. Therefore, the diffusive/anti-diffusive limit equation, i.e., Equation (9), is a particular case of Equation (11). This fact in the case $\sigma=-1$ we state in the following:

Corollary 1. Let $\gamma>1, \sigma=-1$ as well as $f_{0}$ be a probability density on $\mathbb{R}^{d}$ such that $f_{0} \in X^{(4)}$. Let $f^{(\varepsilon)}=f^{(\varepsilon)}(t)$ defined on $[0, T] \times \mathbb{R}^{d}$ be solutions of Equation (4) with the initial datum $f_{0}$ and with $\beta$ given by (6). If $f=f(t)$ in $X, t \in[0, T]$, is a solution to Equation (7) with the same initial datum, on $[0, T] \times \mathbb{R}^{d}$, then

$$
\sup _{[0, T]}\left\|f^{(\varepsilon)}-f\right\| \leq C \varepsilon^{2}
$$

with some constant $C>0$.

Proof. It is sufficient to put $\bar{\beta}(v)=\frac{1}{\omega_{d}} \chi(|v| \leq 1)$. This is a probability density. Then the covariance matrix $\Sigma_{\bar{\beta}}$ is proportional to the identity matrix $I$ and the matrix operation $\Sigma_{\bar{\beta}} \cdot D^{2} f$ provides $\frac{c_{d}}{\omega_{d}} \Delta f$. It is seen that Equation (7) is a particular case of Equation (17).

\section{Numerical Examples}

The properties of the solutions to the considered equations can be illustrated by some numerical examples. Take $d=1, \gamma>1$ and $\sigma= \pm 1$. Since Equation (7) is a limit equation for Equation (4), one can apply some numerical methods such as the finite differences or the method of lines

$$
\partial_{t} f(t, u)=\frac{\sigma}{6}\left(f^{\gamma}(t, u) \frac{f(t, u+\varepsilon)-2 f(t, u)+f(t, u-\varepsilon))}{\varepsilon^{2}}-f(t, u) \frac{\left.f^{\gamma}(t, u+\varepsilon)-2 f^{\gamma}(t, u)+f^{\gamma}(t, u-\varepsilon)\right)}{\varepsilon^{2}}\right)
$$

with $\varepsilon>0$. As a matter of fact, this is a continuous version of the method of lines. For $\sigma=-1$ this is an approximation of a porous-media PDE, which is a parabolic equation. One can investigate the convergence.

If we rearrange Equation (19), it turns out that it coincides with an agent-based model corresponding to kinetic equations

$$
\partial_{t} f(t, u)=\frac{\sigma}{6}\left(f^{\gamma}(t, u) \frac{f(t, u+\varepsilon)+f(t, u-\varepsilon))}{\varepsilon^{2}}-f(t, u) \frac{\left.f^{\gamma}(t, u+\varepsilon)+f^{\gamma}(t, u-\varepsilon)\right)}{\varepsilon^{2}}\right) .
$$

One can see that the solutions to (20) preserve non-negativity of the initial datum as well as $L^{1}$-norm. In both cases $\sigma=-1$ and $\sigma=1$ one can pass to the limit with $\varepsilon \rightarrow 0$. Its derivation resembles the diffusive limit for (4).

Example 1. We consider the initial datum in the form

$$
f_{0}(u)=\left\{\begin{array}{cc}
C \exp \left(-\frac{1}{1-u^{2}}\right), & u \in(-1,1) \\
0, & u \notin(-1,1)
\end{array},\right.
$$

where $C>0$ is a normalizing coefficient. The initial datum $f_{0} \in X$ is an even function and has a single global maximum at $u=0$ (see Figure 1). Then the solution to the method of lines (19) has unique global maximum at zero. 


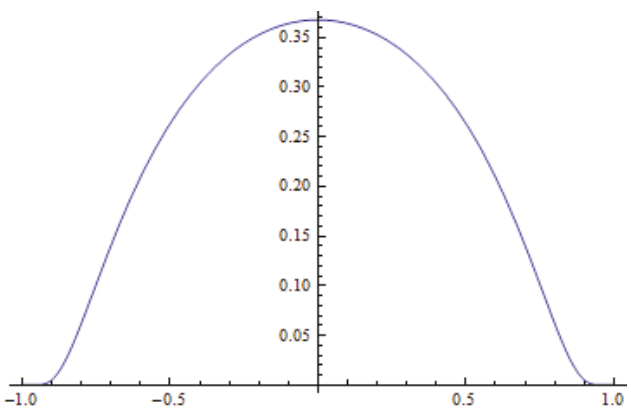

Figure 1. Initial function $f_{0}(u)=C \exp \left(\frac{-1}{1-u^{2}}\right)$ for $u \in(-1,1)$.

Please note that

$$
f(t, u) \approx f_{0}(u)+\left.t \partial_{t} f(t, u)\right|_{t=0} .
$$

If the time derivative is negative in a neighborhood of zero and $t>0$ (see Figure 2), then the maximum of the initial function is transformed into a smaller value (see Figure 3). Therefore, the solution to (19) with $\sigma=-1$ is dispersive. Otherwise, the maximum of the solution to (19) is increasing $(\sigma=1$, see Figures 4 and 5), and the solutions are not dispersive. The time derivative of the solution for $t=0$ does not have any positive twin peaks in the neighborhood of $u=0$ (see Figure 4), which indicates that the solution for anti-diffusion is unipolar. This follows from the fact that the initial datum and its derivatives satisfy the conditions given in [4] (cf. Theorem 3.1).

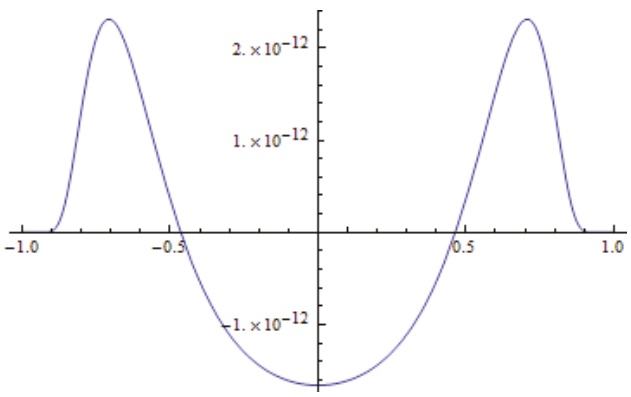

Figure 2. Time derivative $\partial_{t} f(t, u)$ for $t=0, \sigma=-1$ with $f_{0}(u)=C \exp \left(\frac{-1}{1-u^{2}}\right)$ for $u \in(-1,1)$.

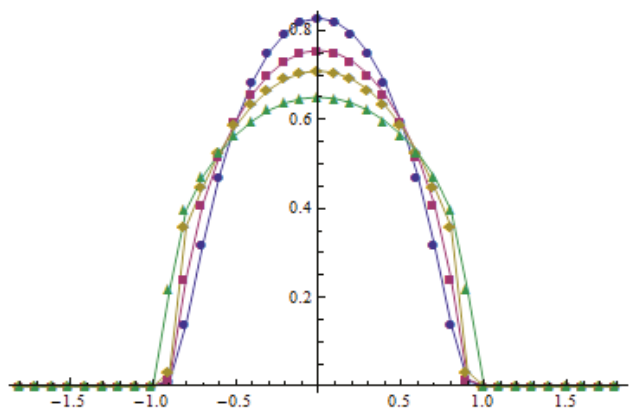

Figure 3. Method of lines for $\varepsilon=10^{-1}, f_{0}(u)=C \exp \left(\frac{-1}{1-u^{2}}\right), \sigma=-1 ; t=0.0$ (blue), $t=0.5$ (red), $t=1.0$ (yellow), $t=2.0$ (green). 
Consider $\sigma= \pm 1$. It follows from Proposition 1 that for $\sigma=-1$ the $L^{1}$-norm and non-negativity of the solution are preserved. The same properties remain for $\sigma=1$. The method of lines for $\sigma=-1$ is stable, unlike for $\sigma=1$. The evolution of the solution is presented at Figures 3 and 5 .

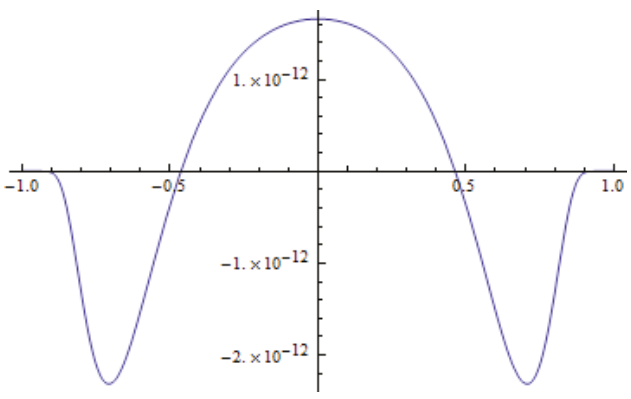

Figure 4. Time derivative $\partial_{t} f(t, u)$ for $t=0, \sigma=1$ with $f_{0}(u)=C \exp \left(\frac{-1}{1-u^{2}}\right)$ for $u \in(-1,1)$.

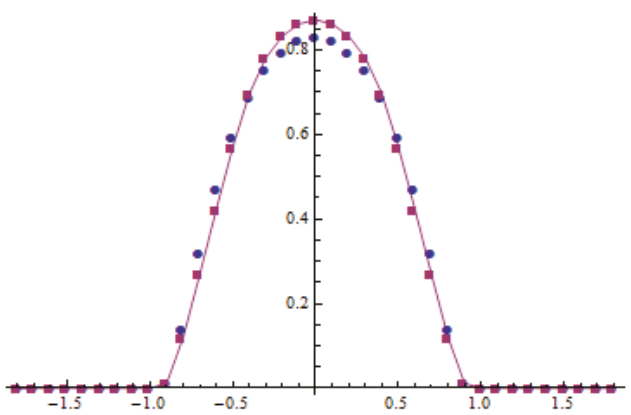

Figure 5. Method of lines for $\varepsilon=10^{-1}, f_{0}(u)=C \exp \left(\frac{-1}{1-u^{2}}\right), \sigma=1 ; t=0.0$ (blue), $t=0.2$ (red).

Example 2. We consider the following initial datum

$$
f_{0}(u)=\left\{\begin{array}{cl}
C\left(1+u^{2}\right) \exp \left(-\frac{1}{1-u^{2}}\right), & u \in(-1,1) \\
0, & u \notin(-1,1)
\end{array},\right.
$$

where $C>0$ is the normalizing coefficient. The initial datum has a single peak in the middle of the interval (see Figure 6), moreover, which has the second order derivative that is equal to zero in $u=0$ (see Figure 7). Therefore, the initial datum is "weakly" concave. The behavior of $f_{0}$ and its second order derivative is presented in Figures 6 and 7. One can see that the initial datum satisfies conditions given in [4] (see Theorem 3.1). Therefore, the method of lines for anti-diffusion transforms the unipolar initial function into the bipolar solution with twin peaks which move away from zero. 


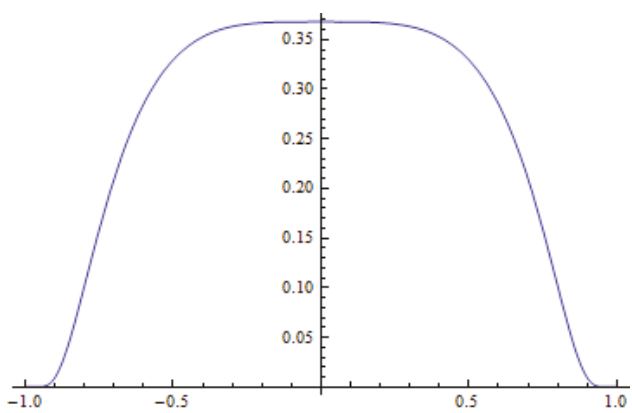

Figure 6. Initial function $f_{0}(x)=C\left(1+u^{2}\right) \exp \left(\frac{-1}{1-u^{2}}\right)$ for $u \in(-1,1)$.

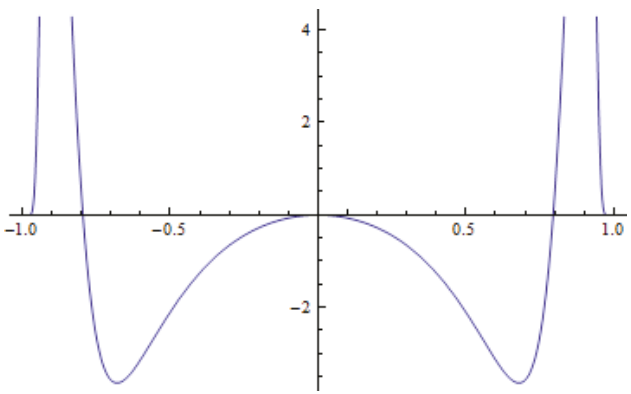

Figure 7. Derivative $f_{0}^{\prime \prime}$ for $f_{0}(u)=C\left(1+u^{2}\right) \exp \left(\frac{-1}{1-u^{2}}\right)$ for $u \in(-1,1)$.

The bipolarity can also be observed in the context of the time derivative for the initial function. The initial function in "weakly" concave in $u=0$. The time derivative for $\sigma=1$ and $t=0$ has symmetric positive twin peaks (see Figure 8). Using the formula (21) one can observe that the values of the time derivative given in the twin peaks added to the values of the initial function in the same points are significantly increasing the value of the initial function. Therefore, the bipolar behavior is observed (see Figure 9).

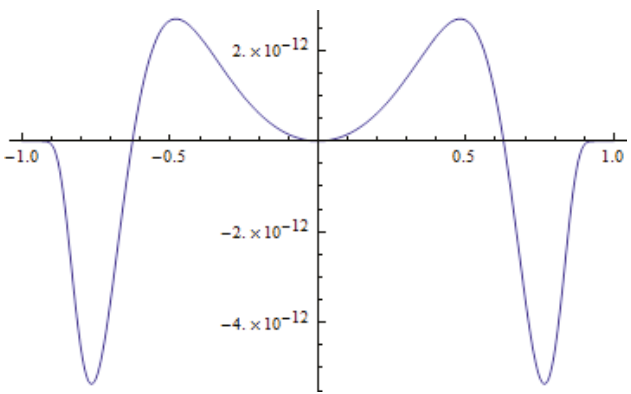

Figure 8. Derivative $\partial_{t} f(t, u)$ for $t=0, \sigma=1$ with $f_{0}(u)=C\left(1+u^{2}\right) \exp \left(\frac{-1}{1-u^{2}}\right)$ for $u \in(-1,1)$. 


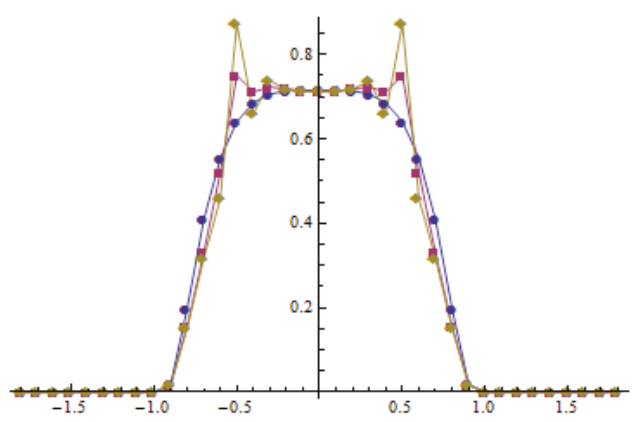

Figure 9. Method of lines for $\varepsilon=10^{-1}, f_{0}(u)=C\left(1+u^{2}\right) \exp \left(\frac{-1}{1-u^{2}}\right), \sigma=1 ; t=0.0$ (blue), $t=0.2$ (red), $t=0.24$ (yellow).

Considering $\sigma=-1$, the time derivative is presented in Figure 10, and the dispersive evolution is shown in Figure 11.

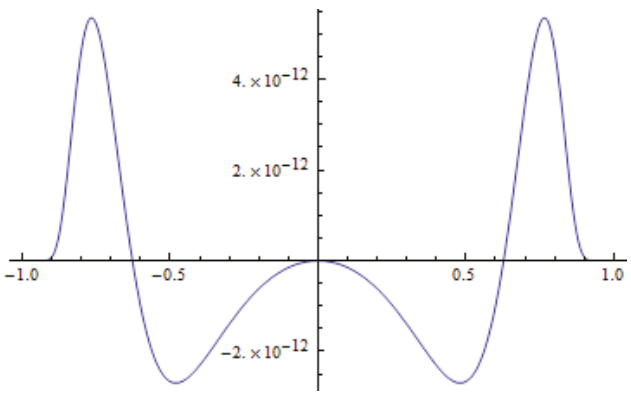

Figure 10. Derivative $\partial_{t} f(t, u)$ for $t=0, \sigma=-1$ with $f_{0}(u)=C\left(1+u^{2}\right) \exp \left(\frac{-1}{1-u^{2}}\right)$ for $u \in(-1,1)$.

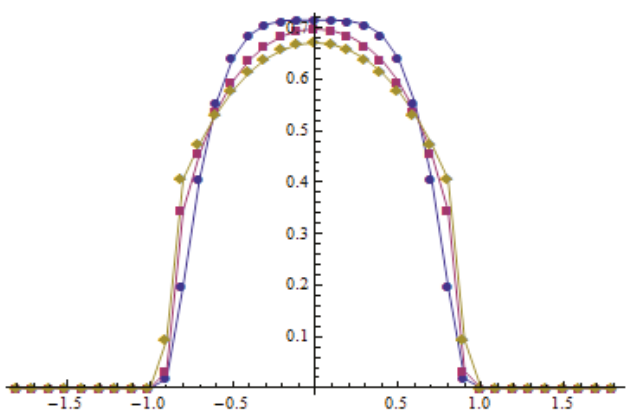

Figure 11. Method of lines for $\varepsilon=10^{-1}, f_{0}(u)=C\left(1+u^{2}\right) \exp \left(\frac{-1}{1-u^{2}}\right), \sigma=-1 ; t=0.0$ (blue), $t=0.5$ (red), $t=1.0$ (yellow).

As before, for $\sigma=-1$, the $L^{1}$-norm and non-negativity of the solution are preserved. The same properties remain for $\sigma=1$. The method of lines for $\sigma=-1$ is stable, unlike for $\sigma=1$. The evolution of the solution to the method of lines for $\sigma=\mp 1$ is presented in Figures 9 and 11.

Our numerical experiments with the method of lines applied to kinetic models in social sciences indicate the possibility of a reliable approximation of short-range interaction opinions in the case of non-conformist societies $(\sigma=-1)$. It has a natural interpretation that the model with short interactions is replaced by a discrete model with discrete opinions influencing neighboring opinions. This replacement is constrained by appropriate scaling according to diffusive limits. Concerning the 
conformist society case $(\sigma=1)$, our numerical simulations show numerical instability. However, we observed the unipolar and bipolar phenomenon on a very small time interval.

\section{Conclusions}

In the paper we studied a class of nonlinear integro-differential kinetic equations that can describe the opinion dynamics for two types of societies - the conformist $\sigma=1$ and anti-conformist $\sigma=-1$. An anti-diffusive (concentration) and a diffusive (dispersive) macroscopic pictures were observed, respectively.

The solutions to the kinetic Equation (4) are a priori positive and preserve $L^{1}$-norm in both considered cases. For $\sigma=-1$ the existence result is global, whereas for $\sigma=1$ it is only local (see Proposition 1), which can be related to a strong concentration (self-organization) in the latter case.

Assuming that $\beta$ (given by (6)) belongs to $L^{1} \cap L^{\infty}, \sigma= \pm 1, \gamma>1$, we conclude that the solutions to the kinetic equation are convergent in $X$ to the solution of a diffusion or (formally) anti-diffusion equations.

In Ref. [4] some bipolar phenomena are described for the kinetic Equation (4). It is a very well-known phenomenon in political sciences (see [4] and references therein).

The present paper opens new interesting problems related to the description of concentration-dispersive phenomena on the mesoscopic scale that may be related to various processes observed in life and social sciences.

Author Contributions: The authors equally contributed to every part of this article.

Funding: M. Lachowicz was supported by the National Science Centre, Poland: Grant 2017/25/B/ST1/00051.

Conflicts of Interest: The authors declare no conflict of interest.

\section{References}

1. Parisot, M.; Lachowicz, M. A kinetic model for the formation of swarms with nonlinear interactions. Kinet. Relat. Model. 2016, 9, 131-164. [CrossRef]

2. Lachowicz, M.; Leszczyński, H.; Parisot, M. A simple kinetic equation of swarm formation: Blow-up and global existence. Appl. Math. Lett. 2016, 57, 104-107. [CrossRef]

3. Lachowicz, M.; Leszczyński, H.; Parisot, M. Blow-up and global existence for a kinetic equation of swarm formation. Math. Model. Methods Appl. Sci. 2017, 27, 1153-1175. [CrossRef]

4. Lachowicz, M.; Leszczyński, H.; Topolski, K.A. Self-organization with small range interactions: Equilibria and creation of bipolarity. Appl. Math. Comput. 2019, 343, 156-166. [CrossRef]

5. Marsan, G.A.; Bellomo, N.; Gibelli, L. Stochastic evolutionary differential games toward a system theory of behavioral social dynamics. Math. Model. Methods Appl. Sci. 2016, 26, 1051-1093. [CrossRef]

6. Banasiak, J.; Lachowicz, M. Methods of Small Parameter in Mathematical Biology; Birkhäuser: Boston, MA, USA, 2014.

7. Lachowicz, M. Individually-based Markov processes modeling nonlinear systems in mathematical biology. Nonlinear Anal. Real World Appl. 2011, 12, 2396-2407. [CrossRef]

8. Banasiak, J.; Lachowicz, M. On a macroscopic limit of a kinetic model of alignment. Math. Model. Methods Appl. Sci. 2013, 23, 2647-2670. [CrossRef]

9. Bellomo, N.; Soler, J. On the mathematical theory of the dynamics of swarms viewed as complex systems. Math. Model. Methods Appl. Sci. 2012, 22, 1140006. [CrossRef]

10. Carrillo, J.A.; Fornasier, M.; Toscani, G.; Vecil, F. Particle, Kinetic, and Hydrodynamic Models of Swarming. In Mathematical Modeling of Collective Behavior in Socio-Economic and Life Sciences; Naldi, G., Pareschi, L., Toscani, G., Eds.; Birkhäuser: Boston, MA, USA, 2010; pp. 297-336.

11. Carrillo, J.A.; D'Orsogna, M.R.; Panferov, V. Double milling in self-propelled swarms from kinetic theory. Kinet. Relat. Model. 2009, 2, 363-378. [CrossRef]

12. Carlen, E.; Degond, P.; Wennberg, B. Kinetic limits for pair-interaction driven master equation and biological swarm models. Math. Model. Methods Appl. Sci. 2013, 23, 1339-1376. [CrossRef] 
13. Degond, P.; Frouvelle, A.; Raoul, G. Local stability of perfect alignment for a spatially homogeneous kinetic model. J. Stat. Phys. 2014, 157, 84-112. [CrossRef]

14. Aronson, D.G. The porous medium equation. In Nonlinear Diffusion Problems; Fasano, A., Primicerio, M., Eds.; Lecture Notes in Mathematics; Springer: Berlin/Heidelberg, Germany, 1986; Volume 1224; pp. 1-46.

15. Vázquez, J.J. The Porous Medium Equation: Mathematical Theory; Oxford University Press: Oxford, UK, 2007.

16. Bénilan, P.; Crandall, M.G.; Pierre, M. Solutions of porous medium equation in $\mathbb{R}^{N}$ under optimal conditions on initial values. Indiana Univ. Math. J. 1984, 33, 51-87. [CrossRef]

17. Lachowicz, M.; Wrzosek, D. Nonlocal bilinear equations: Equilibrium solutions and diffusive limit. Math. Model. Methods Appl. Sci. 2001, 11, 1393-1409. [CrossRef]

18. Benilan, P.; Brezis, H.; Crandall, M.G. A semilinear equation in $L_{1}\left(\mathbb{R}^{N}\right)$. Ann. Scuola Norm. Sup. Pisa 1975, 2, 523-555.

19. Barbu, V. Nonlinear Semigroups and Differential Equations in Banach Spaces; Noordhoff: Leyden, IL, USA, 1976.

(C) 2019 by the authors. Licensee MDPI, Basel, Switzerland. This article is an open access article distributed under the terms and conditions of the Creative Commons Attribution (CC BY) license (http:/ / creativecommons.org/licenses/by/4.0/). 

Article

\title{
Forecasting Efficient Risk/Return Frontier for Equity Risk with a KTAP Approach-A Case Study in Milan Stock Exchange
}

\author{
Marina Dolfin ${ }^{1,2, *,+}$, Leone Leonida ${ }^{2,+}$ and Eleonora Muzzupappa ${ }^{2,+}$ \\ 1 Department of Engineering, University of Messina, 98166 Messina ME, Italy \\ 2 Business School, King's College London, London WC2B 4BG, UK \\ * Correspondence: marina.dolfin@kcl.ac.uk \\ + These authors contributed equally to this work.
}

Received: 1 August 2019; Accepted: 14 August 2019; Published: 16 August 2019

check for updates

\begin{abstract}
We introduce and discuss a dynamics of interaction of risky assets in a portfolio by resorting to methods of statistical mechanics developed to model the evolution of systems whose microscopic state may be augmented by variables which are not mechanical. Statistical methods are applied in the present paper in order to forecast the dynamics of risk/return efficient frontier for equity risk. Specifically, we adopt the methodologies of the kinetic theory for active particles (KTAP) with stochastic game-type interactions and apply the proposed model to a case study analyzing a subset of stocks traded in Milan Stock Exchange. In particular, we evaluate the efficient risk/return frontier within the mean/variance portfolio optimization theory for 13 principal components of the Milan Stock Exchange and apply the proposed kinetic model to forecast its short-term evolution (within one year). The model has the aim to pave the way to many different research perspectives and applications discussed eventually in the paper. In particular, the case of efficient frontier obtained by minimizing the Conditional Value-at-Risk $(\mathrm{CVaR})$ is introduced and a preliminary result is proposed.
\end{abstract}

Keywords: Efficient frontier; kinetic theory; CVaR

\section{Introduction}

The interest in the measurement and mathematical modelization of market risk is strongly supported by the information that it can provide to market stability and portfolio management in general. In fact, the global financial crisis of 2007-2008 raised many issues about prudential regulations and witnessed the importance of resorting to mathematical modeling to test and eventually verify regulatory rules as those of Basel Accords [1].

Financial dynamics resemble the scaling laws that characterize physical systems with large numbers of interacting units [2]. It is worth noticing that, as many complex systems, financial systems reflect the dynamic interaction of a large number of interacting agents, resulting in a systemic behaviour difficult to predict and control [3,4].

Many well established models of economic systems apply methods of statistical mechanics and many of them come from the kinetic theory of rarefied gas, thus adopting the Boltzmann Equations [5,6]. In Reference [5], for instance, power law tails of Pareto type has been retrieved for the wealth distribution obtained as quasi-stationary solution and one of the authors introduced kinetic model of welfare dynamics in Reference [7].

Methods of statistical mechanics and of the kinetic theory's approach have been developed by Helbing, who modeled individual interactions by methods of game theory. A unified approach, suitable to link methods of the statistical mechanics, kinetic theory and game theory, is proposed 
in Reference [8], where master equations are derived to provide a quantitative information on the dynamics of many different social systems. Modeling the overall dynamics of modern societies, where hard sciences such as mathematics and physics can contribute to improve the quality of life is one of the main purposes of the scientific research by Helbing and coworkers [9]. A parallel approach can be found in Reference [10], which uses Boltzmann-type equations where the velocity is replaced by internal variables related to the specific social systems under consideration. Moreover, one can refer to a more specific framework of the generalized kinetic theory which is given by the Fokker-Planck approach which has been applied by various authors to modeling social sciences [11,12].

We adopt here the mathematical approach of the kinetic theory of active particles $[13,14]$, according to which the interacting entities are called active particles, in short a-particles. In some applications, the overall system may be clustered into groups of interest called functional subsystems, while the strategy expressed by the a-particles is depicted by an internal variable at the microscopic state called activity which is heterogeneously distributed among them.

The collective state of the system is modeled by a probability distribution over the microscopic state, while the interaction dynamics is modeled by suitable developments of theoretical tools of game theory [15-20], where the main feature is that players are probability distribution and the output of interactions is delivered in probability. Moreover, applications can include space dynamics [21,22].

The development of this approach to modeling social systems, which evolved through various applications to a system approach, has been recently reviewed in References $[13,14]$. We recall here, as significant examples, applications to collective learning towards opinion formation, started in References [23-27]; support-opposition to governments [3,10,28-30] and competition between cells of the immune system and carriers of pathology [31-34].

We cite here Reference [4] which ends with: "The main challenge is whether and to which extent the kinetic approach is suitable for modelling and forecasting these incentives and dynamics." In this paper we propose a basic model within the kinetic theory approach to approach the technical issue of dynamic potfolio theory, which is traditionally solved within the theory of stochastic dynamic programming, suffering, however of the "curse of dimensionality" [35].

The paper continues with four more sections and a short appendix. Section 2 introduces the basics of the Markowitz theory of portfolio management and optimization and in particular the risk/return efficient frontier whose dynamical evolution is analyzed in the paper. Section 3 describes the proposed model by introducing the mathematical structure governing the dynamics of the efficient frontier within the KTAP theory approach. The general model is then applied to a case study in which the efficient frontier is constructed by considering 13 principal components of the Milan Stock Exchange and the model is applied to forecast the time evolution of the risk distribution of 13 optimal portfolios which are derived by optimizing the return, given the risk, within the Markowitz theory approach. Section 5 discusses significant limitations of the model when applied in the context of the Markowitz portfolio theory and introduces a different possible application by considering the efficient frontier evaluated optimizing the Conditional Value-at-Risk (CVaR). A short appendix, briefly describing a fundamental property of the CVaR measure, closes the paper.

\section{The Risk/Return Efficient Frontier in Portfolio Management}

Market risk has been traditionally measured by the variance (or standard deviation) of portfolio returns and credit risk management adopted this point of view as well. We will discuss later in the paper different measures of risk that are now more commonly used in risk management. However, we initially set our model within the classical Markowitz portolio optimization framework [36,37], that is, using variance (or standard deviation) as a measure of risk and discuss in the paper generalizations of a coherent measure of risk [38] (see the Appendix A: CVaR as a coherent measure of risk).

We here briefly recall some basic assumptions of portfolio optimization and, at the same time, set the notation that will be used throughout the paper. 
Suppose having a portfolio of $n$ risky assets whose composition is determined by the weights $w_{i}, i=1, \ldots, n$, normalized such that $\sum_{i=1}^{n} w_{i}=1$. Markowitz portfolio theory characterizes return and risk of the portfolio through a weighted average of the first and second moment, respectively, of the time series of returns of the risky assets. Specifically, if $r_{i}$ is the $i$-th asset mean return and $\sigma_{i j}$ the covariance between any pair of assets $i$ and $j$, the return of the portfolio of risky asset is the sum of the returns of the individual assets, scaled by the weights, that is,

$$
r_{p}=\sum_{i=1}^{n} w_{i} r_{i}
$$

and its variance is

$$
\sigma_{p}^{2}=\sum_{i=1}^{n} \sum_{j=1}^{n} w_{i} w_{j} \sigma_{i j} .
$$

Passing to the matrix notation, the loss is therefore

$$
f(\boldsymbol{w}, \mathbf{r})=-\boldsymbol{w}^{T} \mathbf{r},
$$

where $\mathbf{r}=\left\{r_{1}, \ldots, r_{n}\right\}$ and the superscript indicate the transpose of the vector $\boldsymbol{w}=\left\{w_{1}, \ldots, w_{n}\right\}$. In our modelling setting another important quantity is the Pearson correlation coefficient, which for each pair of assets reads

$$
\rho_{i j}=\frac{\sigma_{i j}}{\sigma_{i} \sigma_{j}},
$$

with $-1<\rho_{i j}<1$ and with $\sigma_{i}=\sigma_{i i}$ variance of the $i$-th asset in the portfolio.

Portfolio theory in the mean-variance approach is mostly based on some basic assumptions; one of them being the "compactness" of the distributions of stock returns, which can be viewed as the continuity of stock prices [39]. Of course, for instance assuming the presence of transaction costs, should mean that skewness and other higher moments shouldn't be entirely ignored. Moreover, some dramatic events determining high percentages of loss in the stock market prices are ruled out. However, in a first approach we will consider that the conditions for mean-variance (or mean-standard deviation) apply. In the research perspectives we will discuss some details about generalizing these quite restrictive assumptions. Another point worth mentioning is that the theory assumes the normality of stock returns distributions [39]. This is not the case usually, but an argument is that even if asset returns are not exactly normal, the distribution of returns of a large portfolio should resemble a normal distribution.

\section{Efficient Frontier}

A main goal of quantitative investment managers and risk managers is to choose the proportions of various assets to be held in a portfolio by using methods of portfolio optimization, which aims to maximize a measure or proxy for a portfolio's return contingent on a measure or proxy for a portfolio's risk [36,37]. Basically, Markowitz theory is based on a portfolio choice problem in which one looks for the minimum risk related to a given level of return or the maximum return related to a given level of risk. Portfolios satisfying these criteria are said to be efficient and the line connecting point in the risk/return plane characterizing the risks and returns of these portfolios forms a curve called the efficient frontier. The risk/return efficient frontier characterizes the risk/return opportunities available to an investor with the minimum variance, once the return of the portfolio is fixed, or, vice versa, with the maximum return, fixed the variance, that is, the risk of the portfolio. For any portfolio below the line of the efficient frontier, there is a portfolio that has the same variance (i.e., "riskiness") but a greater expected return, that is, it is inefficient. 


\section{Statement of the Problem and Derivation of the Mathematical Structure}

In our model we are facing the topic regarding diversification in a portfolio, that is, the case in which investments are made in a wide variety of assets in order to limit the exposure to risk of a particular security.

Due to the fact that our focus is on diversification, we simplify our analysis to the case of idiosyncratic risk without considering systemic risk, which is non-diversifiable.

We derive a mathematical structure to model the resulting collective dynamics of $n$ efficient portfolios of assets by adopting the KTAP approach $[13,14]$. The mathematical structure is the discretization of a class of integro-differential equations modelling the evolution of the probability distribution over the microscopic state of the interacting entities. By assuming the Markowitz modelling framework we characterize risk by the variance and return by the mean and, in particular, each asset represented by an a-particle possesses a microscopic characteristic representing its variance (asset volatility). The variance at the current time is evaluated from the historical time series of the market prices of the asset.

Following Reference [39], we adopt the point of view that the proper way to assess the risk of an individual asset is through its impact on the volatility of the entire portfolio.

A portfolios' riskiness is discretized, so that it assumes $n$ integer values $I=\{i=1, \ldots, n\}$, ranging from the portfolio with the lowest risk to the one with the highest risk (assuming a reasonable minimal and maximal value). As a consequence, we have chosen a discrete activity variable as a natural choice in this problem in which the activity variable is going to characterize the 'riskiness' of a finite and discrete number of efficient portfolios.

The overall state of the system is described, according to the KTAP approach, by the discrete probability (see Reference [7] for instance for an application in wealth distribution):

$$
f(t)=\left\{f_{i}(t)\right\}, \quad f:[0, T] \times I \rightarrow[0,1],
$$

representing the (normalized) distribution of risk of a given number of efficient portfolios, say $n$ portfolios, and satisfying the following constraint

$$
\sum_{i=1}^{n} f_{i}(t)=1, \quad \forall t \in[0, T]
$$

for a fixed time interval $[0, T]$.

From an applicative point of view, given a set of assets, we use their historical time series of market prices, and solve $n$ optimization problems that allows one to choose $n$ optimal portfolios among the feasible set of portfolios, each of them characterized by an increasing level of riskiness. The optimal portfolios are then the efficient ones, that is, each of them is maximizing the return given the risk (the vice versa situation of minimizing the risk, given the return follows an analogous procedure).

By balancing in the elementary volume of the space of micro-state the inlet and the outlet fluxes one gets the following system of ordinary differential equations (ODEs):

$$
\frac{d f_{i}}{d t}=J_{i}[f]=\sum_{h, k=1}^{n} \mathcal{B}_{h k}^{i}[f] \eta_{h k} f_{h} f_{k}-f_{i} \sum_{k=1}^{m} \eta_{i k} f_{k}, \quad i=1,2 \ldots, n,
$$

with the initial condition

$$
f\left(t_{0}\right)=\left\{f_{i 0}\right\}, \quad f_{i 0}: I \rightarrow[0,1], \quad i=1,2 \ldots, n .
$$

An appropriate interpretation of the phenomenology is needed in order to derive the appropriate explicit expression of the following quantities: 
- $\quad \eta_{h k}$ - the interaction rate, that is, the frequency of interactions of the test a-particle with a field a-particle;

- $\quad \mathcal{B}_{h k}^{i}$ - the probability that the test a-particle in the $h$-th risk state shifts to the $i$-th risk state due to an encounter with a field a-particle in the $k$-th risk state.

Transition probabilities satisfy the following natural constraints

$$
\begin{aligned}
& \mathcal{B}_{h k}^{i} \geq 0, \quad \forall i, h, k, \\
& \sum_{i=1}^{n} \mathcal{B}_{h k}^{i}=1, \quad \forall h, k,
\end{aligned}
$$

and the encounter rate is such that

$$
\eta_{h k} \geq 0 \quad \forall h, k
$$

Modelling the Dynamics of Risky Assets in a Portfolio

To derive the model for the dynamics of portfolios' riskiness, we need to trasfer the interpretation of the phenomenology of the observed system into a heuristic specification for the transition probabilities and the interaction rates. Then, by introducing the explicit terms into the general mathematical structure (7) we get the desired model.

To set the explicit epression for the transition probabiliies, we have chosen an approach borrowing tools derived from game theory [15-20], and assuming a stochastic outcome for any binary interaction, typically related to the presence of risk, that is, to uncertainty.

The hallmarks that we use to model the transition probability density that characterizes the probability of the interacting agents to shift their riskiness status, that is, to change (by lowering or rising) their riskiness, are illustrated in the following.

When a pair of assets "interacts", i.e., they belong to the same efficient portfolio, the output of the interaction determines a (possible) increase in riskiness if the correlation coefficient between them is positive and a (possible) decrease in the riskiness if the correlation coefficient is negative. Eventually, if the two assets are uncorrelated, there is no shift in the portfolio risk to which they belong. The frequency of interactions depends on the weights of the interacting a-particle in each portfolio. Then, we can say that each a-particle, that is, each asset in the portfolio, has binary interactions with the other a-particles, delivering a hiding-chasing stochastic game driven by the correlations between each pair of involved a-particles.

According to the above guidelines, the transition probabilities are constructed by distinguishing among three cases:

1. Interactions between uncorrelated a-particles $\left(\rho_{h k}=0\right)$, or of an a particle with itself $(h=k)$ :

$$
\left\{\begin{array}{l}
\mathcal{B}_{h k}^{i=h}=1 \\
\mathcal{B}_{h k}^{i \neq h}=0
\end{array} \quad \forall i, h, k=1, \ldots, n\right.
$$

2. Interactions between positively correlated a-particles $\left(\rho_{h k}>0\right)$ :

- if $h=n$ then

$$
\left\{\begin{array}{l}
\mathcal{B}_{h k}^{i=n}=1 \\
\mathcal{B}_{h k}^{i \neq n}=0
\end{array} \forall i, h, k=1, \ldots, n\right.
$$


- if $h \neq n$ then

$$
\left\{\begin{array}{l}
\mathcal{B}_{h k}^{i=h+1}=\rho_{h k} \\
\mathcal{B}_{h k}^{i=h}=1-\rho_{h k}, \quad \forall i, h, k=1, \ldots, n \\
\mathcal{B}_{h k}^{i \neq h, h+1}=0
\end{array}\right.
$$

3. Interactions between negatively correlated a-particles $\left(\rho_{h k}<0\right)$ :

- if $h=1$ then

$$
\left\{\begin{array}{l}
\mathcal{B}_{h k}^{i=1}=1 \\
\mathcal{B}_{h k}^{i \neq 1}=0
\end{array} \forall i, h, k=1, \ldots, n\right.
$$

- if $h \neq 1$ then

$$
\left\{\begin{array}{l}
\mathcal{B}_{h k}^{i=h-1}=-\rho_{h k} \\
\mathcal{B}_{h k}^{i=h}=1+\rho_{h k}, \quad \forall i, h, k=1, \ldots, n \\
\mathcal{B}_{h k}^{i \neq h-1, h}=0
\end{array}\right.
$$

It is straightforward to verify that the above introduced transition probabilities verify the properties $(9,10)$. In proposing the basic model, binary interactions are assumed to be linear, depending on the correlation coefficient which is assumed to be the constant one measured at the current time. However, a research perspective would be that of considering that the correlation coefficient between any pair of assets updates with the time evolution of the portfolio, maybe leading to a more precise forecasting.

The encounter rate $\eta_{h k}$ determines the frequency of interactions of pairs of a-particles, that is, of assets belonging to the same portfolio. Among different possibilities to model it, we have chosen to apply the following reasoning: the frequency of interactions roughly speaking "weights "the effect of one asset with respect to another one in the portfolio and then, we model the encounter rate through the average of the weights of the interacting asset in the different portolios of the efficient frontier, that is,

$$
\eta_{h k}=\mathbb{E}\left[\boldsymbol{w}_{h}\right], \quad \forall k=1, \ldots, n,
$$

where the vector $w_{h}$ characterizes the weights of the $h$-th asset, each component representing the weight in the corresponding efficient portfolio of the risk-return frontier. Then, for instance the first component of $w_{h}$ is the weight of the $h$-th asset in the first portfolio of the efficient frontier. It is worth noticing that by adopting this viewpoint, the frequency of interaction depends only on the test a-particle (with index $h$ in this case).

\section{A Case Study on Principal Components of Milan Stock Exchange}

To derive a short-term evolution of the risk profile of $n$ efficient portfolios we use as initial condition in the mathematical structure (7) the (normalized) frequency distribution of risk obtained by applying the Markowitz portfolio optimization, with the simplifying constraint of no short positions allowed (i.e., $w_{i} \geq 0, i=1, \ldots, n$ ), on the following randomly chosen 13 stocks traded in the Milan Stock Exchange (Table 1): 
Table 1. Stocks of the case study.

\begin{tabular}{cc}
\hline Ticker & Stock \\
\hline CPR.MI & Davide Campari-Milano S.p.A. \\
SRG.MI & Snam S.p.A. \\
UNI.MI & Unipol Gruppo S.p.A. \\
AZM.MI & Azimut Holding S.p.A. \\
FCA.MI & Fiat Chrysler Automibiles NV \\
REC.MI & Recordati Industria Chimica e Farmaceutica S.p.A. \\
SFER.MI & Salvatore Ferragamo S.p.A. \\
TRN.MI & Tenaris S.A. \\
MONC.MI & Moncler S.p.A. \\
UBI.MI & Unione di Banche Italiane S.p.A. \\
JUVE.MI & Juventus Football Club S.p.A. \\
BPE.MI & BPER Banca S.p.A. \\
ENI.MI & Eni S.p.A. \\
\hline
\end{tabular}

We used monthly historical time series of prices for the above listed 13 components of the FTSE.mib over a time period of 5 years, ranging from 30/06/2014 to 28/06/2019, using 61 time recording of the market prices for the above introduced 13 stocks. The histograms of distributions of logarithmic returns are summarized in Figure 1:
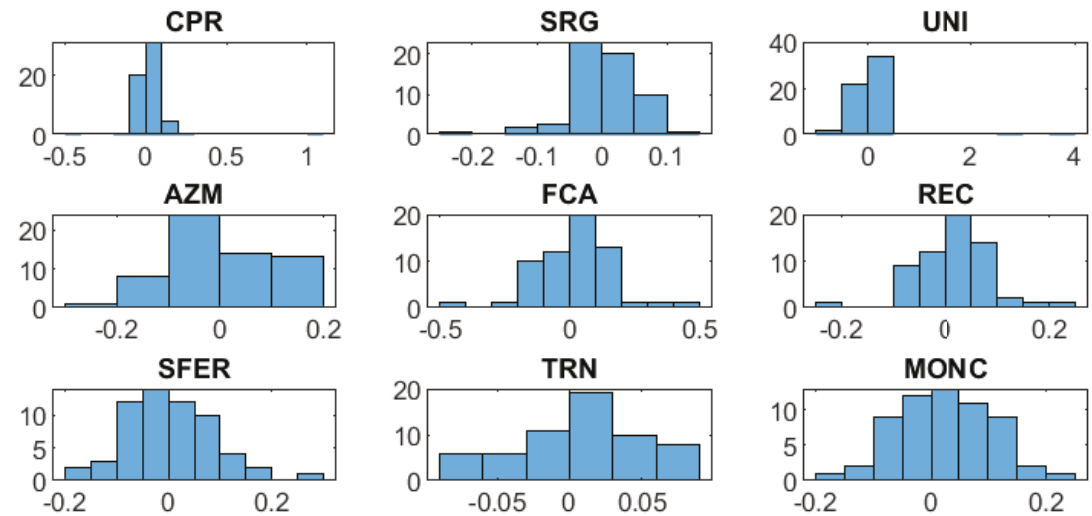

MONC
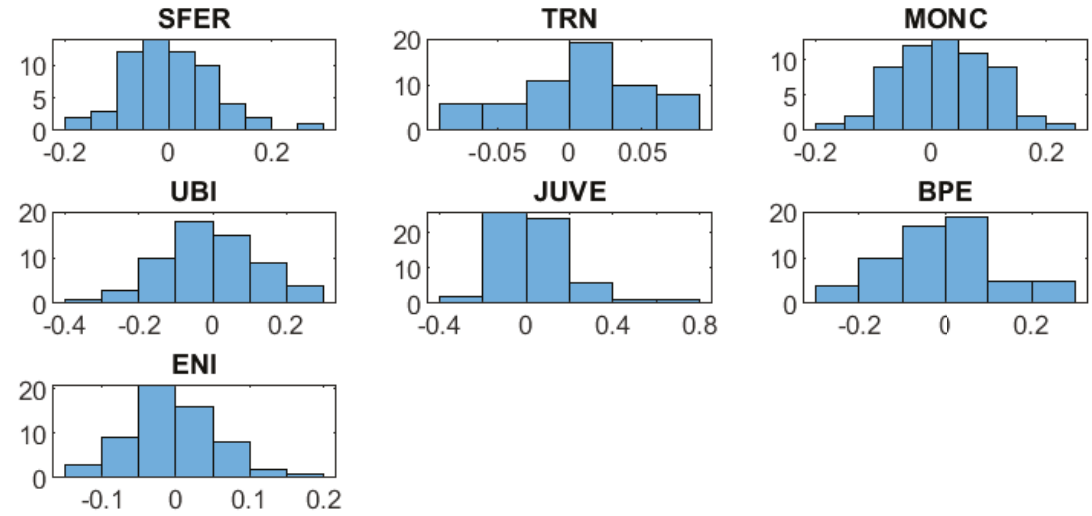

Figure 1. Logarithmic return distributions for each stock of the panel data (logarithmic return on the abscissa and frequency on the ordinate).

where for each stock the related histogram represents the logaritmic return of the asset (i.e., $\ln \left(\frac{P_{t}}{P_{t-1}}\right)$ with $P_{t}$ price at time $t$ ) on the market (in the abscissa) and the corresponding frequency of observation. 
Among them, we derive the weights of the portfolio maximizing the Sharpe ratio $S R=\frac{r_{p}-r_{f}}{\sigma_{p}}$, with $r_{f}$ risk-free rate of return. The risk and return of this portfolio is a point in the risk/return plane, indicated by the yellow star in Figure 2. The risk free rate $r_{f}$ has been fixed at 0.01 . Although the risk proxy in mean-variance is the variance of portfolio returns, the standard deviation of portfolio returns is usually displayed, and we adopt the same notation here.

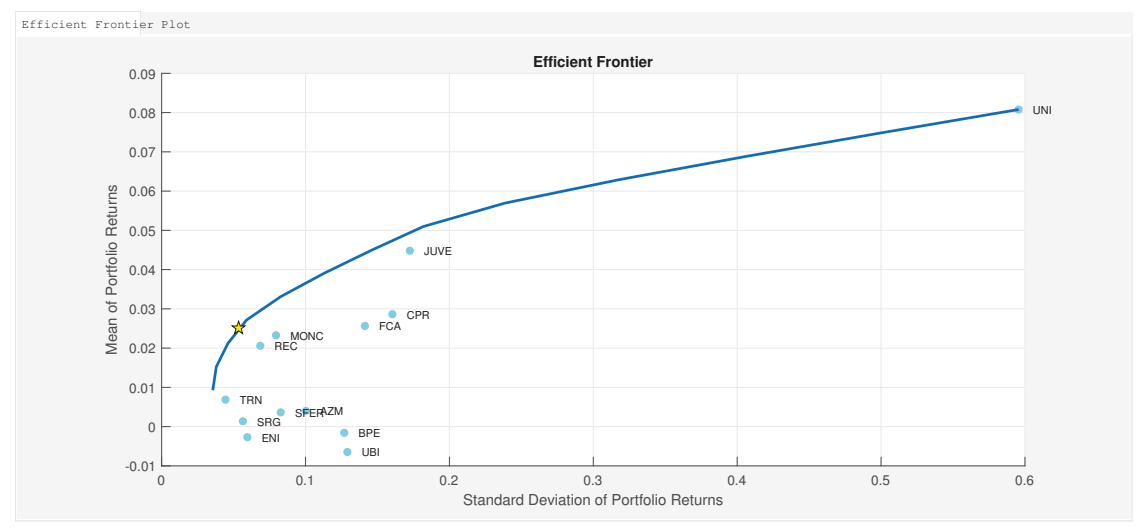

Figure 2. Efficient frontier for the given panel of data evaluated on 13 portfolios.

\section{Results and Discussion}

We use the risk distribution obtained by the mean-variance optimization performed on the given panel data of stock returns as initial data in the system of Equation (7) with the proposed modellization of the probabilities of transition and encounter rate. The vector of correlation coefficients for each pair of asset has been evaluated through the covariance matrix obtained from the historical time series of the panel data for the 13 stocks of the Milan Stock Exchange given in Table 1 using a time window of 5 years. The initial condition (8) representing the risk profile for the 13 efficient portfolios obtained with the 13 stocks is graphically represented on the left of Figure 3 below. Once evaluated the matrices of transition probabilities and encounter rate, we plug them into the system of ODEs (7). A graphical representation of the solution at time $T=10$ (months) is showed on the right part of the figure.

Moreover, in this section we discuss future research directions within the framework of our model and propose some preliminary numerical experiments.

\section{Basel Committee Capital Accords and the CVaR as Risk Measure}

Basel Accords [1] try to define rules in order to evaluate the capital necessary to support a bank's portfolio. Basically, regulators need to ensure clear rules to measure the minimal capital requirements. The first document has been introduced in 1988 and then revisioned in Basel II and Basel III and set the guidelines and rules for measuring, managing and reporting the banks' risk exposures, fundamentally requiring the use of analytical approaches.

The evalution of minimal capital requirements depends on the calculation of the probability distribution of the portfolio loss. In fact, the equity capital allocated to the portfolio must be equal to the percentile of the distribution of the portfolio loss that corresponds to the desired probability (e.g., about 0.001 for the AA quality). Credit risk modelling has the goal to obtain an estimate of the risk, expressed as statistics (usually quantiles) of the potential loss distribution within a chosen time period (in the financial industry, it is usually taken as one year). At the basis of Basel Accords there is the model introduced by Vasicek [40]. 

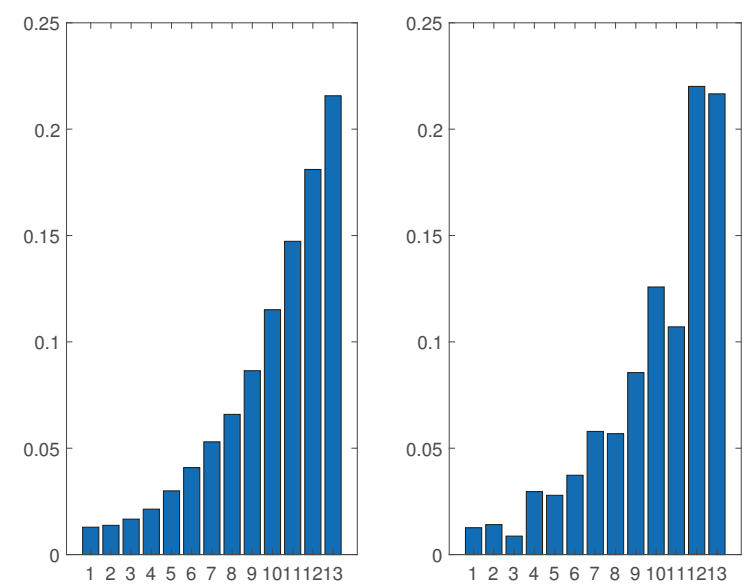

Figure 3. Risk distribution for the 13 efficient portfolios of the risk-return frontier (on the abscissa) of the case study with the mean-variance optimization at time $t_{0}$ (left) and its evolution at the time horizon $T=10$ (months) (right).

There exist different measures to evaluate potential losses. Value-at-Risk (VaR), for instance, calculates the maximum potential loss at a certain confidence level (e.g., a $97.5 \%$ VaR measures a loss that is expected to be exceeded only $2.5 \%$ of the time), while Conditional Value-at-Risk (CVaR) evaluates the average loss above the same confidence level (e.g., a 97.5\% CVaR measures the average of the worst $2.5 \%$ of losses). VaR calculates then the losses at a single threshold in the distribution (e.g., 97.5\%), whereas CVaR averages all losses exceeding the thereshold in the distribution. For this reason, $\mathrm{CVaR}$ is more efficient than VaR measures to capture the tail risks. As a consequence, even if the same threshold is used, the value of CVaR is higher than the value of VaR. The importance of this point is expecially relevant in presence of fat-tailed distributions. As a consequence, in the revised risk management framework, the 97.5 th percentile CVaR is roughly equivalent to the 99th percentile VaR used in Basel 2.5.

Measures of risk can be used as (extra) capital requirements to regulate the risk, however, how to measure financial risk is a long debated topic with primary focus on coherence properties [38] of the proposed quantities, in particular subaddittivity (see the Appendix B at the end of the paper).

The Basel Committee has proposed to substitute the VaR with the CVaR in the regulatory framework on market risks. Having this in mind, in the following we show the graphical representation of the efficient frontier obtained by minimizing the CVaR [41] to make the comparison with the mean/variance efficient frontier, for the proposed panel of data. To describe the probability distribution of returns we used a finite sample of return scenarios $y_{s}, s=1, \ldots, S$, where each $y_{s}$ is a vector that contains the returns for each of the $n$ assets under the scenario $s$ [41]. The efficient frontiers obtained with the mean-variance optimization (dashed-line) and with the CVaR optimization (solid line) are showed in Figure 4. Moreover, we graphically compare the weights for the 13 portfolios obtained with the two different approaches in Figure 5. 


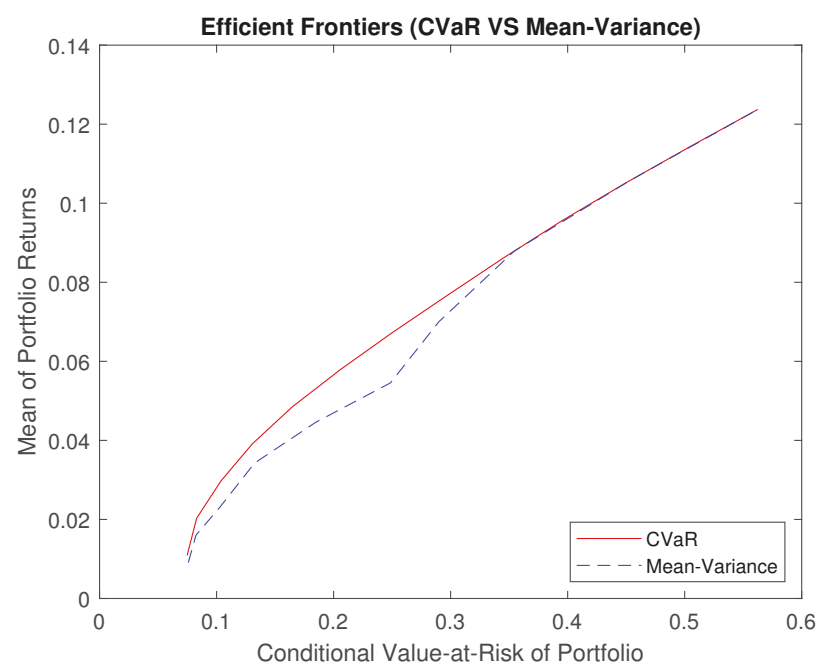

Figure 4. Conditional Value-at-Risk (CVaR) vs mean-variance optimization for the given panel of data.
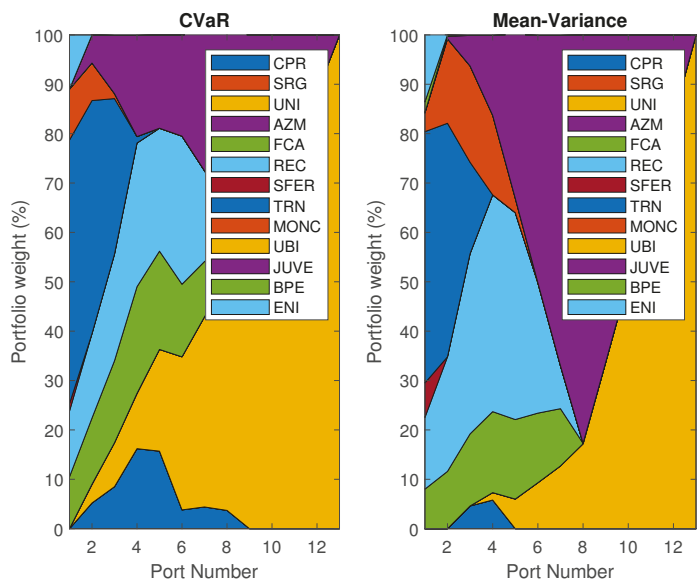

Figure 5. Portfolio weights of CVaR VS Mean-Variance optimization for the given panel of data.

We now use the risk distribution obtained by the CVaR optimization on the same panel of stock returns as initial data in the system of Equation (7), with the proposed modellization of the transition probabilities and encounter rate and, of course, the same vector of correlation coefficients as previously. The initial condition (8) representing the risk profile for the 13 efficient portfolios obtained with the 13 stocks is graphically represented on the left of Figure 6 below. We then apply the some procedure as in the case of the mean-variance efficient frontier. A graphical representation of the solution at time $T=10$ (months) is showed on the right part of Figure 6.

We have proposed the model in its simplest form, in order to express the underlying logic. It is worth noticing, however, that the KTAP approach is suitable to be applied in a more general way, allowing the better management of the heterogeneity of the different efficient portfolios. In particular, the concept of functional subsystem, applied by two of the authors of Reference [30], may be usefully applied to enhance the effect of the heterogeneity of the portfolios and of possible network interactions 
among them. Another research perspective might be that of introducing non linear transition probabilities by considering that the correlation coefficient between any pair of assets updates with the time evolution of the portfolio, so that it will depend of the probability distribution of the activity variable itself. This source of nonlinearity would allow the model to be more consistent with real-world features of the market. Introducing a continuous probability distribution for the activity variable representing risk would also lead to interesting research perspectives, mainly from a mathematical point of view. Finally, we just notice here that we have simply chosen randomly the basket of stocks among the 30 principal components of the Milan Stock Exchange. However, basing on the theory of portfolios' management, we imagine that the more the distribution of returns of a stock differs from a normal distribution, the more the two methods based on VaR or CVaR differ. Of course, this simple intuition has to be supported by quantitative measurements and we plan to investigate in this direction in a next future.
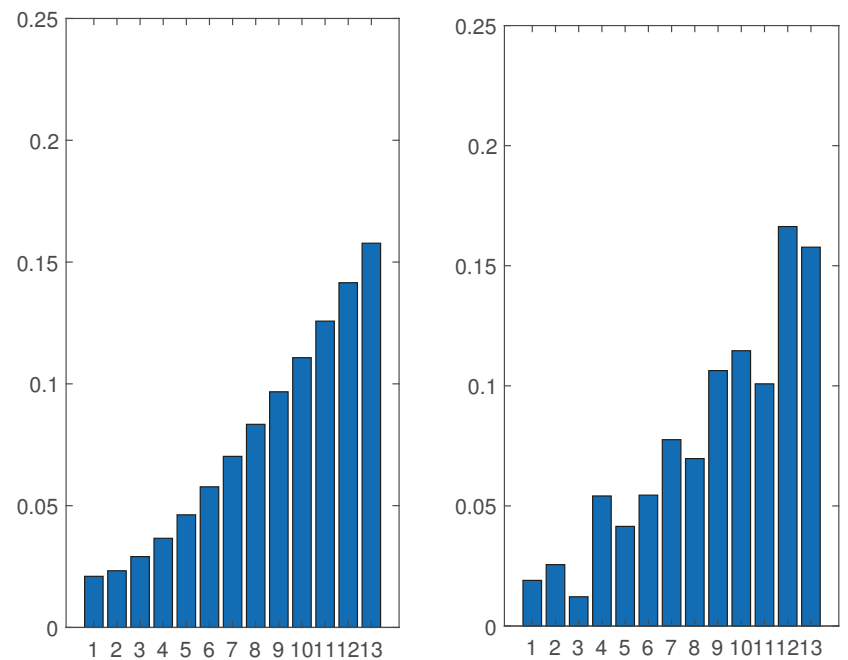

Figure 6. Risk distribution for the 13 efficient portfolios of the risk-return frontier (on the abscissa) of the case study with the CVaR optimization at time $t_{0}$ (left) and its evolution at the time horizon $T=10$ (months) (right).

Author Contributions: Conceptualization, M.D., L.L. and E.M.; Methodology, M.D.; Supervision, L.L. and E.M.; Writing-original draft, M.D., L.L. and E.M.

Funding: This research received no external funding.

Acknowledgments: The authors wish to thank the anonymous referees for their very valuable suggestions that allowed us to increase the quality of the final version of the paper.

Conflicts of Interest: The authors declare no conflict of interest.

\section{Appendix A. CVaR as a Coherent Measure of Risk}

Conditional Value-at-Risk, known also as Expected shortfall (ES), is a coherent measure of risk used to evaluate the credit risk of a portfolio. CVaR at the $\alpha \%$ level, is the portfolio return in the worst $\alpha \%$ of cases, that is, the focus is on the less profitable outcomes. If we consider a set $V$ of real-valued random variables, a risk measure is a real-valued function $\rho: V \rightarrow \mathbb{R}$. Sub-addittivity provides that

$$
\text { if } X, Y, X+Y \in V \Rightarrow \rho(X+Y) \leq \rho(X)+\rho(Y)
$$


with the simple meaning of "a merger does not create risk" [38]. The global risk of a portfolio will always be less than the sum of its partial risks due to diversification, unless it is equal when it is triggered by concurrent events. In any case it is not "natural" the possibility that the global risk is higher than the sum of the partial risks. In Reference [42] one can find the simple example of a bank made of several branches; once the capital requirement of each branch is evaluated, the regulator has to be confident that also the overall bank capital is an adequate one, without allowing the possibility that the whole bank risk turn out to be much bigger than the sum of the branches' risks. Moreover, a measure of risk should consider the entire shape of the tail of losses beyond the VaR [43], such as in the case of $\mathrm{CVaR}$

$$
C V a R_{\alpha}=\mathbb{E}\left\{X \mid X>V a R_{\alpha}\right\}
$$

measuring the mean of losses conditional to the exceedence of the VaR. One could also express the CVaR with the analytical formula

$$
C V a R_{\alpha}=\frac{1}{1-\alpha} \int_{\alpha}^{1} \operatorname{VaR}_{\beta} d \beta
$$

so that the CVaR with $\alpha$ level of confidence is the average of all the VaR with $\beta>\alpha$ confidence.

\section{Appendix B. Materials and Methods}

All data and computer codes associated with the publication are available to readers. Monthly historical series of prices have been downloaded from the website Yahoo Finance (https://it.finance.yahoo.com). Efficient frontiers, both using the mean-variance approach and the $\mathrm{CVaR}$ portfolio optimization, have been evaluated using the Financial Toolbox and the Optimization Toolbox of Matlab. The Cauchy's problem given by the ODEs (7), with the transitions probabilities and encounter rate (12)-(17) and with the initial conditions (8) given by the risk of the efficient portfolios, has been solved using the Forward Euler method for differential equations. All graphs have been plotted using Matlab.

\section{References}

1. Basel Committee on Banking Supervision 2005. International Convergence of Capital Measurement and Capital Standards, a Reviewed Framework. Available online: https://www.bis.org/publ/bcbs128.htm (accessed on 9 June 2019).

2. Lux, T.; Marchesi, M. Scaling and criticality in a stochastich multi-agent model of a financial market. Nature 1999, 397, 498-500. [CrossRef]

3. Dolfin, M.; Leonida, L.; Outada, N. Modeling human behavior in economics and social science. Phys. Life Rev. 2017, 22-23, 1-21. [CrossRef] [PubMed]

4. Kapetanios, G.; Muzzupappa, E. Regulatory capture and financial crisis. Phys. Life Rev. 2017, $22-23,44$. [CrossRef] [PubMed]

5. Cordier, S.; Pareschi, L.; Toscani, G. On a kinetic model for a simple market economy. J. Stat. Phys. 2005, 120, 263-277. [CrossRef]

6. Düring, B.; Pareschi, L.; Toscani, G. Kinetic models for optimal control of wealth inequalities. Eur. Phys. J. B 2018, 91, 265. [CrossRef]

7. Dolfin, M.; Lachowicz, M. Modeling altruism and selfishness in welfare dynamics: the role of nonlinear interactions. Math. Model. Methods Appl. Sci. 2014, 24, 2361-2381. [CrossRef]

8. Helbing, D. Quantitative Sociodynamics. Stochastic Methods and Models of Social Interaction Processes, 2nd ed.; Springer: Berlin/Heidelberg, Germany, 2010.

9. Helbing D.; Yu W. The outbreak of cooperation among success-driven individuals under noisy conditions. Proc. Natl. Acad. Sci. USA 2009, 106, 3680-3685. [CrossRef] [PubMed]

10. Pareschi, L.; Toscani, G. Interacting Multiagent Systems: Kinetic Equations and Monte Carlo Methods; Oxford University Press: Oxford, UK, 2013. 
11. Furioli, G.; Pulvirenti, A.; Terraneo, E.; Toscani, G. Fokker-Planck equations in the modeling of socio-economic phenomena. Math. Model. Methods Appl. Sci. 2017, 27, 115-158. [CrossRef]

12. Gualandi, S.; Toscani, G. Call center service times are lognormal: A Fokker-Planck description. Math. Model. Methods Appl. Sci. 2018, 8, 1513-1527. [CrossRef]

13. Ajmone Marsan, G.; Bellomo, N.; Gibelli, L. Stochastic evolutionary differential games toward a systems theory of behavioral social dynamics. Math. Model. Methods Appl. Sci. 2016, 26, 1051-1093. [CrossRef]

14. Bellomo, N.; Knopoff, D.; Soler, J. On the difficult interplay between life, "complexity" and mathematicl sciences. Math. Model. Methods Appl. Sci. 2013, 23, 1861-1913. [CrossRef]

15. Allen, B.; Nowak, M.A. Games on Networks. EMS Surv. Math. Sci. 2013, 1, 113-151. [CrossRef]

16. Camerer, C.F. Behavioral Game Theory: Experiments in Strategic Interaction; Princeton University Press: Princeton, NJ, USA, 2003.

17. Gintis, H. Game Theory Evolving, 2nd ed.; Princeton University Press: Princeton, NJ, USA, 2009.

18. Hofbauer, J.; Sigmund, K. Evolutionary game dynamics. Bull. Am. Math. Soc. 2003, 40, 479-519. [CrossRef]

19. Nash, J. Essentials of Game Theory; Elgar: Cheltenham, UK, 1996.

20. Nowak, M.A. Evolutionary Dynamics. Exploring the Equations of Life; Harward University Press: Cambridge, MA, USA, 2006.

21. Bellomo, N.; Bellouquid, A.; Knopoff, D. From the microscale to collective crowd dynamics. Multiscale Model. Simul. 2013, 11, 943-963. [CrossRef]

22. Bellomo, N.; Ha, S.-Y. A quest toward a mathematical theory of the dynamics of swarms. Math. Model. Methods Appl. Sci. 2017, 27, 745-770. [CrossRef]

23. Brugna, C.; Toscani, G. Kinetic models of opinion formation in the presence of personal conviction. Phys. Rev. E 2015, 92, 052818.. [CrossRef]

24. Burger, M.; Caffarelli, L.; Markowich, P. Partial differential equation models in the socio-economic sciences. Phylos. Trans. R. Soc. 2014, 372, 20130406. [CrossRef]

25. Burini, D.; De Lillo, S.; Gibelli, L. Collective learning modeling based on the kinetic theory of active particles. Phys. Life Rev. 2016, 16, 123-139. [CrossRef]

26. Dolfin, M.; Lachowicz, M. Modeling opinion dynamics: how the network enhances consensus. Netw. Heterogeneous Med. 2015, 10, 877-896. [CrossRef]

27. Toscani, G. Kinetic models of opinion formation. Commun. Math. Sci. 2006, 4, 481-496. [CrossRef]

28. Bellomo, N.; Herrero, M.; Tosin, A. On the dynamics of social conflicts: looking for the black swan. Kinet. Relat. Models 2013, 6, 459-479.

29. Bertotti, M.L.; Modanese, G. From microscopic taxation and redistribution models to macroscopic income distributions. Physica A 2011, 390, 3782-3793. [CrossRef]

30. Dolfin, M.; Knopoff, D.; Leonida, L.; Maimone Ansaldo Patti, D. Escaping the trap of 'blocking': A kinetic model linking economic development and political competition. KInet. Rel. Mod. 2017, 10, 423-443. [CrossRef]

31. Bellouquid, A.; Delitala, M. Modelling Complex Biological Sytems: A Kinetic Theory Approach, Series: Modeling and Simulation in Science, Engineering and Technology; Birkhäuser: Boston, Switzerland, 2006.

32. Bellouquid, A.; De Angelis, E.; Knopoff, D. From the modeling of the immune hallmarks of cancer to a black swan in biology. Math. Model. Methods Appl. Sci. 2013, 23, 949-978. [CrossRef]

33. De Angelis, E. On the mathematical theory of post-Darwinian mutations, selection and evolution. Math. Model. Methods Appl. Sci. 2014, 24, 2723-2742. [CrossRef]

34. De Lillo, S.; Delitala, M.; Salvatori, M. Modelling epidemics and virus mutations by methods of the mathematical kinetic heory for active particles. Math. Model. Methods Appl. Sci. 2009, 19, 1405-1425. [CrossRef]

35. Brandt, M.W. Portfolio Choice Problems. In Handbook of Financial Econometrics; Ait-Sahalia, Y., Hansen, L.P., Eds.; Elsevier: Oxford, UK, 2010; pp. 269-336.

36. Markowitz, H.M. Portfolio Selection. J. Financ. 1952, 7, 77-91.

37. Markowitz, H.M. Portfolio Selection: Efficient Diversification of Investments. John Wiley \& Sons, Inc.: Hoboken, NJ, USA, 1959.

38. Artznerm, P.; Delbaen, F.; Eber, J.-M.; Heat, D. Coherent measures of risk. Math. Financ. 1999, 9, $203-228$. [CrossRef] 
39. Bodie, Z.; Kane, A.; Marcus, A.J. Investments and Portfolio Management; The Mac-Graw-Hill Europe: New York, NY, USA, 2010.

40. Vasicek, O. The Distribution of Loan Portfolio Value. Risk 2002, 15, 160-172.

41. Rockafellar, R.T.; Uryasev, S. Optimization of conditional Value-at-risk. J. Risk 1998, 2, 21-24. [CrossRef]

42. Acerbi, C.; Scandolo, G. Liquidity risk theory and coherent measures of risk. Quant. Financ. 2008, 8, 681-692. [CrossRef]

43. Christoffersen, P.F. Elements of Financial Risk Management; Academic Press: Cambridge, MA, USA, 2003.

(C) 2019 by the authors. Licensee MDPI, Basel, Switzerland. This article is an open access article distributed under the terms and conditions of the Creative Commons Attribution (CC BY) license (http:/ / creativecommons.org/licenses/by/4.0/). 
Article

\title{
Numerical Simulation of a Multiscale Cell Motility Model Based on the Kinetic Theory of Active Particles
}

\author{
Damián A. Knopoff ${ }^{1, *}$, Juanjo Nieto ${ }^{2}$ and Luis Urrutia ${ }^{2}$ \\ 1 Universidad Nacional de Córdoba and CIEM (CONICET), 5000 Córdoba, Argentina \\ 2 Departamento de Matemática Aplicada, Universidad de Granada, 18071 Granada, Spain \\ * Correspondence: damian.knopoff@unc.edu.ar
}

Received: 12 June 2019; Accepted: 4 July 2019; Published: 3 August 2019

check for updates

\begin{abstract}
In this work, we deal with a kinetic model of cell movement that takes into consideration the structure of the extracellular matrix, considering cell membrane reactions, haptotaxis, and chemotaxis, which plays a key role in a number of biological processes such as wound healing and tumor cell invasion. The modeling is performed at a microscopic scale, and then, a scaling limit is performed to derive the macroscopic model. We run some selected numerical experiments aimed at understanding cell movement and adhesion under certain documented situations, and we measure the alignment of the cells and compare it with the pathways determined by the extracellular matrix by introducing new alignment operators.
\end{abstract}

Keywords: multiscale modeling; cell movement; haptotaxis; kinetic theory

\section{Introduction}

Studying and understanding cell movement is crucial in biological sciences and medicine, since it is essential to a variety of biological processes such as morphogenesis, wound healing, cancer metastasis, and immune response, among others [1]. Haptotaxis is understood as the movement of the cells due to interactions with the substances that form the extracellular matrix (ECM), and it is influenced by its structure. Depending on the cell type, its size, and if the migration is individual or collective, we can find different types of haptotactic movements [2,3]. Haptotaxis occurs either due to the presence of an oriented compound on the ECM, like a filament or a proteic fiber, or by a difference in the concentration of certain chemical compounds that stick to the ECM and promote binding of cells. The latter might be seen as a similar mechanism to chemotaxis; however, chemotaxis should not be seen as a special case of haptotaxis [4]. One of the structures that appears when studying the haptotactic movement of cells is the integrins, cell membrane adhesion proteins that bind with the substances on the ECM $[5,6]$.

On the other hand, chemotaxis is a process by which cells change their state of movement reacting to the presence of a certain free chemical compound in the surrounding media of the cell population. Usually, cells are affected by both phenomena simultaneously, even it is possible to be caused by the same substance on different states. This is the case of thrombospondin 1, forcing both haptotaxis and chemotaxis on the movement of melanoma cells [7-9].

Both processes, chemotaxis and haptotaxis, constitute directional mobilities of cells whose driving forces are gradients: for haptotaxis, a gradient of cellular adhesion sites, while for chemotaxis, a chemical concentration gradient in a soluble fluid. These gradients are naturally present in the ECM of the body during processes such as angiogenesis or artificially-induced in biomaterials where gradients are established by altering the concentration of adhesion sites on a polymer substrate. 
The binding of cells to the ECM and the different processes that modify the state of the integrins have been studied from a mathematical point of view. We refer the reader to [10] for some models focused on cell reactions involving these compounds. In [11], these reactions were incorporated into a kinetic model for cell motility, adding the concentration of integrins as a new microscopic variable, taking the main role in promoting the movement of the cellular population.

In the literature, chemotaxis has received much more attention than haptotaxis, starting with the classical model due to Patlak [12], and Keller and Segel in the 1970s [13]. The mathematical properties of this classical model for chemotaxis, in particular the blow-up of the solutions for finite time, have led to the development of new ways of studying chemotaxis from a mathematical point of view, many of them modifying the aforesaid model. The survey by Hillen and Painter [14] collected several different variations of this classical model, and they incorporated new mathematical terms modeling biological phenomena, such as nonlinear diffusion, non-constant sensibility to the chemoattractant, and control of the population by direct or indirect ways, among others. These models can also be deduced from a microscopic point of view by using the tools of hydrodynamical limits $[15,16]$.

More recent results on this challenging topic were reviewed in the surveys [17,18], while some recent achievements have enlightened the role of anomalous diffusion $[19,20]$ and of the environment where the dynamics develops [21]. Indeed, our paper considers models in the complex environment constituted by the ECM, where cells move.

On the other hand, the development of mathematical models studying haptotaxis is relatively recent. Starting with the first model of Oster et al. in the early 1980s [22], early models for haptotactic movement were proposed as a particular case of chemotaxis with a non-diffusing signal. Later, in [23], the authors introduced both the adhesion of cells to the ECM, as well as the cell-membrane integrins, responsible for the aforementioned adhesion and promoters of cell migration. Recent works [11,24] have two important differences from the previous ones: first, the modeling was done through a kinetic approach to haptotaxis; second, the directionality of the structural compounds of the ECM, and not the gradient of said compounds, took the main role in cell motility.

There exists a wide literature related to the mathematical modeling of cell motility, which due to the biological nature of the phenomena, involves an appreciable complexity. One of the most visited topics is the modeling of the invasion of healthy tissues by tumors. In particular, we remark about the contribution by Chaplain et al. [25], where a complete model containing haptotaxis due to the ECM, chemotaxis due to different chemical compounds in the surrounding media, and interactions between all the involved cells was deduced for the cancer cell invasion of a tissue. In this line, many more rigorous and numerical approaches have been tackled (see [23,24,26-28] among others) by using kinetic and/or macroscopic models, each of them with its own mathematical complexity.

It is worth mentioning that the mathematical modeling of biological phenomena and, in particular, of the dynamics of multicellular systems has recently taken advantage of the framework delivered by the so-called Kinetic Theory of Active Particles (KTAP) [29]. The main difference of the KTAP approach with respect to the classical kinetic theory to model living systems is that the microscopic state of the interacting entities, which are called active particles, includes, in addition to mechanical variables, another variable called activity, which models biological functions specific for each system under consideration. This is the approach used in the present paper, by highlighting that the activity variable allows us, in contrast with other approaches, to consider several microscopic biological variables as independent variables, and not as unknowns, whose dynamics is well defined, obtaining an important reduction of the complexity of the final model.

Some specific tools of the KTAP approach have so far been used in many other applications. For instance, competition between tumor and immune cells [30] and social dynamics [31,32], crowd dynamics [17,18,33-36], and swarming [37]. Thus, the contents of this paper may be useful for the modeling of other phenomena in life sciences. Notice that the modeling of these specific systems needs a deep understanding of the interactions between living entities, as in the case of cell dynamics, where the "living behavior" belongs to the specific functions expressed by cells. Mathematical models 
require the application of computational methods appropriate for capturing the specific features of multi-agent systems. Monte Carlo particle methods have shown the ability to handle this challenging problem of numerical analysis [38].

Finally, let us mention some specific applications on the derivation of macroscopic equations from the underlying description at the microscopic scale delivered by kinetic theory models. Various types of regular and singular perturbation methods have been applied for different types of models, as examples in vehicular traffic [34], as well as in the dynamics of cells [20,21,39]. The unified approach of a method inspired to the Hilbert problem was developed in [40], while a general reference was given by [41].

The paper is organized as follows. Section 2 presents the kinetic model for the movement of a cell population whose physical-biological properties were described in $[42,43]$ and performs the hyperbolic scaling for the underlying description at the micro- and meso-scopic scales. Section 3 is devoted to the numerical scheme, and three case-studies are proposed and analyzed with special insight into a measure of alignment for the adaptation of cells to the ECM. Finally, Section 4 presents the conclusions and some research perspectives.

\section{Description of the Model}

This section is devoted to the description of the model presented in [43] and the hyperbolic scaling. The model describes the movement of a cell population in $\mathbb{R}^{N}$ and the evolution of two chemical compounds, each one related to one of the processes described before: an oriented protein fiber, denoted by $Q(t, x, \theta)$, where $\theta \in \mathbb{S}^{N-1}$ represents its orientation, and a degenerated chemical $L(t, x)$, responsible for chemotaxis. The density of proteic fibers at time $t$ and position $x$ is denoted by $\bar{Q}(t, x)$ :

$$
\bar{Q}(t, x):=\int_{\mathbb{S}^{N-1}} Q(t, x, \theta) d \theta .
$$

We describe the cell population by means of a distribution function $f(t, x, v, y)$ depending on time $t$, space $x$, velocity $v$, and activity $y$ (which will be described below), verifying the following equation deduced in [42],

$$
\frac{\partial f}{\partial t}+v \cdot \nabla_{x} f+\nabla_{y} \cdot(G(y, \bar{Q}, L) f)=\mathcal{H}(f, Q)+\mathcal{L}(f)+\mathcal{C}(f, L),
$$

where the right-hand side models the cell mobility by way of velocity changes and the $y$-divergence term is related to the cell membrane reactions. Concretely,

- the term $\mathcal{H}$, modeling haptotaxis, is:

$$
\begin{aligned}
\mathcal{H}(f, Q)(t, x, v, y) & :=\int_{V} \int_{\mathbb{S}^{N-1}} p_{h}\left(t, x, v^{\prime}, y\right) \psi\left(v ; v^{\prime}, \theta\right) f\left(t, x, v^{\prime}, y\right) Q(t, x, \theta) d \theta d v^{\prime} \\
& -p_{h}(t, x, v, y) f(t, x, v, y) \int_{V} \int_{\mathbb{S}^{N-1}} \psi\left(v^{\prime} ; v, \theta\right) Q(t, x, \theta) d \theta d v^{\prime} ;
\end{aligned}
$$

- the turning operator $\mathcal{L}$ models random changes in velocity,

$$
\begin{aligned}
\mathcal{L}(f)(t, x, v, y) & :=\int_{V} p_{l}\left(t, x, v^{\prime}, y\right) \alpha_{1}(y) T\left(v, v^{\prime}\right) f\left(t, x, v^{\prime}, y\right) d v^{\prime} \\
& -p_{l}(t, x, v, y) \alpha_{1}(y) f(t, x, v, y) \int_{V} T\left(v^{\prime}, v\right) d v^{\prime}
\end{aligned}
$$

- $\quad$ and the chemotactic term, $\mathcal{C}$, reads:

$$
\begin{aligned}
\mathcal{C}(f, L)(t, x, v, y) & :=\int_{V} p_{c}\left(t, x, v^{\prime}, y\right) \alpha_{2}(y) K[\nabla L]\left(v, v^{\prime}\right) f\left(t, x, v^{\prime}, y\right) d v^{\prime} \\
& -p_{c}(t, x, v, y) \alpha_{2}(y) f(t, x, v, y) \int_{V} K[\nabla L]\left(v^{\prime}, v\right) d v^{\prime} .
\end{aligned}
$$


Here, $p_{h}, p_{l}$, and $p_{c}$ are the interaction frequencies, $\psi, T$ and $K$ are the interaction kernels, and $\alpha_{i}$ are nonnegative weight functions satisfying $\alpha_{1}+\alpha_{2}=1$. $V$ is the domain of velocities, which is given by the velocities verifying $0<s_{1} \leq|v| \leq s_{2}$.

In order to define the activity $y$ and the cell membrane reaction terms, we need to recover the law of mass action of the reactions produced at the cell membrane involving the two chemicals in the ECM and the receptors on the cell,

$$
\bar{Q}+R \underset{k_{-1}}{\stackrel{k_{1}}{\rightleftharpoons}} \bar{Q} R, \quad L+R \underset{k_{-2}}{\stackrel{k_{2}}{\rightleftharpoons}} L R,
$$

where $R$ stands for the free enzyme on the cell surface and $\bar{Q} R$ and $L R$ represent the respective complexes once the enzyme binds the ECM chemical. Then, $y$ is defined as the two-component vector of microscopic concentrations of the two cell-membrane compounds $\bar{Q} R$ and $L R$, respectively. It is defined in the set:

$$
Y=\left\{\left(y_{1}, y_{2}\right) \in\left(0, R_{0}\right) \times\left(0, R_{0}\right): y_{1}+y_{2}<R_{0}\right\},
$$

where $R_{0}>0$ represents the maximum concentration of receptors on the cell surface. The function $G$ is given by the expression:

$$
G(y, q, l):=\left(\begin{array}{l}
k_{1}\left(R_{0}-y_{1}-y_{2}\right) q-k_{-1} y_{1} \\
k_{2}\left(R_{0}-y_{1}-y_{2}\right) l-k_{-2} y_{2}
\end{array}\right)
$$

whose rows represent the equations associated with (2).

Finally, we introduce the macroscopic equations for the free chemicals $Q$ and $L$ in the ECM:

$$
\begin{aligned}
\frac{\partial Q}{\partial t}= & -\kappa\left(\int_{V} \int_{Y}\left(1-\left|\theta \cdot \frac{v}{|v|}\right|\right) f d v d y\right) Q \\
& -k_{1} Q \int_{V} \int_{Y}\left(R_{0}-y_{1}-y_{2}\right) f d v d y+\frac{k_{-1}}{\left|\mathbb{S}^{N-1}\right|} \int_{V} \int_{Y} y_{1} f d v d y
\end{aligned}
$$

and:

$$
\begin{aligned}
\frac{\partial L}{\partial t}= & \kappa \int_{\mathbb{S}^{N-1}}\left(\int_{V} \int_{Y}\left(1-\left|\theta \cdot \frac{v}{|v|}\right|\right) f d v d y\right) Q d \theta-r_{L} L+D_{L} \Delta_{x} L \\
& -k_{2} L \int_{V} \int_{Y}\left(R_{0}-y_{1}-y_{2}\right) f d v d y+k_{-2} \int_{V} \int_{Y} y_{2} f d v d y .
\end{aligned}
$$

\section{The Hyperbolic Scaling}

We have so far referred to macroscopic and microscopic models. However, they are not independent of each other, since they are two different ways of viewing the same reality. If we face the same real situation, whose nature requires different scales, we must describe it by combining models of the two types, which should be related in some way. This is the underlying concept of multiscale modeling: modeling the same real situation with different scales, and their relationship. The relationship between the different scales is given by scaling limit: broadly speaking, it is performed by "zooming out" the window through which we observe a phenomenon, and by transforming a microscopic model into a macroscopic model.

In this subsection, we perform the calculations leading to the nondimensionalization and scaling of the system(1)-(3)-(4) [43].

In the following, the interaction frequencies $p_{h}, p_{l}$, and $p_{c}$ are considered to be constant (otherwise, the scaling does not make sense). First of all, we define the dimensionless ("hat") variables:

$$
\begin{gathered}
t:=\hat{t} \tau, \quad x:=\hat{x} R, \quad v:=\hat{v} s_{2}, \quad y:=\hat{y} R_{0}, \\
f(t, x, v, y):=\bar{f} \hat{f}(\hat{t}, \hat{x}, \hat{v}, \hat{y}), \quad Q(t, x, \theta):=R_{0} \hat{Q}(\hat{t}, \hat{x}, \theta), \quad L(t, x):=R_{0} \hat{L}(\hat{t}, \hat{x}),
\end{gathered}
$$




$$
\begin{gathered}
p_{k}(t, x, v, y):=\bar{p}_{k}(k=h, l, c), \quad G(t, Q, L):=\bar{G} \hat{G}(\hat{y}, \hat{Q}, \hat{L}), \\
\alpha_{j}(y):=\hat{\alpha}_{j}(\hat{y}),(j=1,2), \quad T\left(v, v^{\prime}\right):=\frac{1}{s_{2}^{n}} \hat{T}\left(\hat{v}, \hat{v}^{\prime}\right), \\
\psi\left(v ; v^{\prime}, \theta\right)=\frac{1}{R_{0} s_{2}^{n}} \hat{\psi}\left(\hat{v} ; \hat{v}^{\prime}, \theta\right), \quad K\left(v, v^{\prime}\right):=\frac{1}{s_{2}^{n}} \hat{K}\left(\hat{v}, \hat{v}^{\prime}\right),
\end{gathered}
$$

where $\tau, R, \bar{f}, \bar{p}_{k}$, and $\bar{G}$ are typical quantities of their respective variables. The new variables are defined in the sets:

$$
\hat{V}:=\frac{1}{s_{2}} V, \quad \hat{Y}:=\frac{1}{R_{0}} Y .
$$

Our system then becomes:

$$
\begin{aligned}
& \frac{\partial \hat{f}}{\partial \hat{t}}+\frac{s_{2} \tau}{R} \hat{v} \cdot \nabla_{\hat{x}} \hat{f}+\frac{\tau \bar{G}}{R_{0}} \nabla_{\hat{y}} \cdot(\hat{G} \hat{f})=\bar{p}_{h} \tau \hat{\mathcal{H}}(\hat{f}, \hat{Q})+\bar{p}_{l} \tau \hat{\mathcal{L}}(\hat{f})+\bar{p}_{c} \tau \hat{\mathcal{C}}(\hat{f}, \hat{L}), \\
& \frac{\partial \hat{Q}}{\partial \hat{t}}=-\tau R_{0}^{2} s_{2}^{n} \bar{f} \kappa\left(\int_{\hat{V}} \int_{\hat{Y}}\left(1-\left|\theta \cdot \frac{\hat{v}}{|\hat{v}|}\right|\right) \hat{f} d \hat{v} d \hat{y}\right) \hat{Q} \\
& \quad-\tau R_{0}^{3} s_{2}^{n} \bar{f} k_{1} \hat{Q} \int_{\hat{V}} \int_{\hat{Y}}\left(1-\hat{y}_{1}-\hat{y}_{2}\right) \hat{f} d \hat{v} d \hat{y}+\tau R_{0}^{2} s_{2}^{n} \bar{f} \frac{k_{-1}}{\left|\mathbb{S}^{N-1}\right|} \int_{\hat{V}} \int_{\hat{Y}} \hat{y}_{1} \hat{f} d \hat{v} d \hat{y}, \\
& \frac{\partial \hat{L}}{\partial \hat{t}}= \tau R_{0}^{2} s_{2}^{n} \bar{f} \kappa \int_{\mathbb{S}^{N-1}}\left(\int_{\hat{V}} \int_{\hat{Y}}\left(1-\left|\theta \cdot \frac{\hat{v}}{|\hat{v}|}\right|\right) \hat{f} d \hat{v} d \hat{y}\right) \hat{Q} d \theta-\tau r_{L} \hat{L}+\frac{\tau}{R^{2}} D_{L} \Delta_{\hat{x}} \hat{L} \\
&-\tau R_{0}^{3} s_{2}^{n} \bar{f} k_{2} \hat{L} \int_{\hat{V}} \int_{\hat{Y}}\left(1-\hat{y}_{1}-\hat{y}_{2}\right) \hat{f} d \hat{v} d \hat{y}+\tau R_{0}^{2} s_{2}^{n} \bar{f} k_{-2} \int_{\hat{V}} \int_{\hat{Y}} \hat{y}_{2} \hat{f} d \hat{v} d \hat{y} .
\end{aligned}
$$

We impose first the normalization restrictions $\frac{s_{2} \tau}{R}=1$ and $\frac{\tau}{R^{2}} D_{L}=1$. The hyperbolic scaling corresponds to the choice:

$$
\tau \bar{p}_{l}=\frac{1}{\varepsilon}
$$

i.e., the turning time $\frac{1}{\bar{p}_{l}}$ is very small compared to the typical time $\tau$.

There are three other phenomena (cell membrane reactions, haptotaxis, and chemotaxis) to consider. We rescale the corresponding terms, assuming also that their frequencies are small compared to the turning frequency $\bar{p}_{l}$. More precisely, we choose the following relations:

$$
\frac{\bar{G}}{R_{0}}=\varepsilon^{a} \bar{p}_{l}, \quad \bar{p}_{h}=\varepsilon^{b} \bar{p}_{l}, \quad \bar{p}_{c}=\varepsilon^{d} \bar{p}_{l}
$$

where $0<a<1, b, d \geq 1$.

To scale the other two equations, we recall that they are actually macroscopic, so they will preserve their form. This is why we only define the scaled dimensionless constants involved:

$$
\begin{gathered}
\hat{\kappa}:=\tau R_{0}^{2} s_{2}^{n} \bar{f} \kappa, \quad \hat{r}_{L}:=\tau r_{L \prime} \\
\hat{k}_{i}:=\tau R_{0}^{3} s_{2}^{n} \bar{f} k_{i}, \quad \hat{k}_{-i}:=\tau R_{0}^{2} s_{2}^{n} \bar{f} k_{-i}, \quad(i=1,2) .
\end{gathered}
$$

Skipping the "hat" for the dimensionless variables, our system becomes:

$$
\varepsilon\left(\frac{\partial f_{\varepsilon}}{\partial t}+v \cdot \nabla_{x} f_{\varepsilon}\right)+\varepsilon^{a} \nabla_{y} \cdot\left(G\left(y, Q_{\varepsilon}, L_{\varepsilon}\right) f_{\varepsilon}\right)=\varepsilon^{b} \mathcal{H}\left(f_{\varepsilon}, Q_{\varepsilon}\right)+\mathcal{L}\left(f_{\varepsilon}\right)+\varepsilon^{d} \mathcal{C}\left(f_{\varepsilon}, L_{\varepsilon}\right)
$$


for the cell population, where:

$$
\begin{aligned}
\mathcal{H}\left(f_{\varepsilon}, Q_{\varepsilon}\right)(t, x, v, y) & =\int_{V} \int_{\mathbb{S}^{N-1}} \psi\left(v ; v^{\prime}, \theta\right) f_{\varepsilon}\left(t, x, v^{\prime}, y\right) Q_{\varepsilon}(t, x, \theta) d \theta d v^{\prime}-f_{\varepsilon}(t, x, v, y) \bar{Q}_{\varepsilon}(t, x), \\
\mathcal{L}\left(f_{\varepsilon}\right)(t, x, v, y) & =\int_{V} \alpha_{1}(y) T\left(v, v^{\prime}\right) f_{\varepsilon}\left(t, x, v^{\prime}, y\right) d v^{\prime}-\alpha_{1}(y) f_{\varepsilon}(t, x, v, y), \\
\mathcal{C}\left(f_{\varepsilon}, L_{\varepsilon}\right)(t, x, v, y) & =\int_{V} \alpha_{2}(y) K\left[\nabla L_{\varepsilon}\right]\left(v, v^{\prime}\right) f_{\varepsilon}\left(t, x, v^{\prime}, y\right) d v^{\prime}-\alpha_{2}(y) f_{\varepsilon}(t, x, v, y)
\end{aligned}
$$

and:

$$
\begin{aligned}
\frac{\partial Q_{\varepsilon}}{\partial t}= & -\kappa\left(\int_{V} \int_{Y}\left(1-\left|\theta \cdot \frac{v}{|v|}\right|\right) f_{\varepsilon} d v d y\right) Q_{\varepsilon} \\
& -k_{1} Q_{\varepsilon} \int_{V} \int_{Y}\left(1-y_{1}-y_{2}\right) f_{\varepsilon} d v d y+\frac{k_{-1}}{\left|\mathbb{S}^{N-1}\right|} \int_{V} \int_{Y} y_{1} f_{\varepsilon} d v d y, \\
\frac{\partial L_{\varepsilon}}{\partial t}= & \kappa \int_{\mathbb{S}^{N-1}}\left(\int_{V} \int_{Y}\left(1-\left|\theta \cdot \frac{v}{|v|}\right|\right) f_{\varepsilon} d v d y\right) Q_{\varepsilon} d \theta-r_{L} L_{\varepsilon}+\Delta_{x} L_{\varepsilon} \\
& -k_{2} L_{\varepsilon} \int_{V} \int_{Y}\left(1-y_{1}-y_{2}\right) f_{\varepsilon} d v d y+k_{-2} \int_{V} \int_{Y} y_{2} f_{\varepsilon} d v d y,
\end{aligned}
$$

for the chemicals, where:

$$
V=[s, 1] \times \mathbb{S}^{N-1}, Y=\left\{\left(y_{1}, y_{2}\right) \in(0,1) \times(0,1): y_{1}+y_{2}<1\right\},
$$

and $s:=s_{1} / s_{2}$. In order to close the macroscopic system that will appear, we choose the turning operator:

$$
\mathcal{L}(f):=\lambda \int_{V} f\left(t, x, v^{\prime}, y\right) d v^{\prime}+\beta v \cdot \int_{V} v^{\prime} f\left(t, x, v^{\prime}, y\right) d v^{\prime}-\lambda|V| f(t, x, v, y),
$$

with $\lambda=\beta \frac{1-s^{n+2}}{\left(1-s^{n}\right)(n+2)}$. If we study the equations verified by the moments of $f_{\varepsilon}$,

$$
\rho_{\varepsilon}:=\int_{V} \int_{Y} f_{\varepsilon} d v d y, \quad \rho_{\varepsilon} U_{\varepsilon}:=\int_{V} \int_{Y} v f_{\varepsilon} d v d y, \quad \rho_{\varepsilon} W_{\varepsilon}:=\int_{V} \int_{Y} y f_{\varepsilon} d v d y,
$$

we obtain the following equations:

$$
\begin{aligned}
& \frac{\partial \rho_{\varepsilon}}{\partial t}+\nabla_{x} \cdot\left(\rho_{\varepsilon} U_{\varepsilon}\right)=0, \\
& \frac{\partial\left(\rho_{\varepsilon} U_{\varepsilon}\right)}{\partial t}+\nabla_{x} \cdot\left(\mathbb{P}_{\varepsilon}+\rho_{\varepsilon} U_{\varepsilon} \otimes U_{\varepsilon}\right)=\varepsilon^{b-1} \int_{V} \int_{Y} v \mathcal{H}\left(f_{\varepsilon}, Q_{\varepsilon}\right) d v d y+\varepsilon^{d-1} \int_{V} \int_{Y} v \mathcal{C}\left(f_{\varepsilon}, L_{\varepsilon}\right) d v d y, \\
& \varepsilon \partial_{t} \rho_{\varepsilon} W_{\varepsilon}+\varepsilon \nabla_{x} \cdot \int_{V} \int_{Y} y \otimes v f d v d y+\varepsilon^{a}\left(A_{\varepsilon} W_{\varepsilon}-b_{\varepsilon}\right) \rho_{\varepsilon}=0 .
\end{aligned}
$$

Here, $\mathbb{P}_{\varepsilon}(t, x):=\int_{V} \int_{Y}\left(v-U_{\varepsilon}\right) \otimes\left(v-U_{\varepsilon}\right) f_{\varepsilon} d v d y$, is the pressure tensor, and the matrix $A_{\varepsilon}$ and the vector $b_{\varepsilon}$ are respectively given by:

$$
A_{\varepsilon}:=\left(\begin{array}{cc}
k_{1} \bar{Q}_{\varepsilon}+k_{-1} & k_{1} \bar{Q}_{\varepsilon} \\
k_{2} L_{\varepsilon} & k_{2} L_{\varepsilon}+k_{-2}
\end{array}\right), \quad b_{\varepsilon}=\left(\begin{array}{c}
k_{1} \bar{Q}_{\varepsilon} \\
k_{2} L_{\varepsilon}
\end{array}\right) .
$$

Then, we make $\varepsilon=0$ on (5) to deduce that the limiting distribution function $f_{0}$ has to be on the kernel of the turning operator, i.e., $\mathcal{L}\left(f_{0}\right)=0$, and then, we assume that the solutions are small 
perturbations of it, $f_{\varepsilon}=f_{0}+\varepsilon f_{1}$, hence a Hilbert expansion of $f_{\varepsilon}$ around $f_{0}$. Inserting the explicit form of $f_{0}$ (see [43] for details) into (12)-(13)-(14), we formally obtain the following macroscopic equations:

$$
\begin{aligned}
& \frac{\partial \rho}{\partial t}+\nabla_{x} \cdot(\rho U)=0 \\
& \frac{\partial(\rho U)}{\partial t}+2 \frac{1-s^{n+2}}{(n+2)\left(1-s^{n}\right)} \nabla_{x} \rho=\delta_{b-1} H(\rho, U, Q)+\delta_{d-1} C(\rho, U, L) \\
& \rho W=\frac{\rho}{k_{1} k_{-2} \bar{Q}+k_{-1} k_{2} L+k_{-1} k_{-2}}\left(\begin{array}{c}
k_{1} k_{-2} \bar{Q} \\
k_{-1} k_{2} L
\end{array}\right) .
\end{aligned}
$$

Here, the macroscopic integral operators are defined as:

$$
\begin{aligned}
H(\rho, U, Q) & :=\frac{\rho}{|V|}\left(\int\left(\Psi^{1}(\theta)+\frac{\beta}{\lambda} \Psi^{2}(\theta) \cdot U\right) Q(\theta) d \theta-\bar{Q}|V| U\right), \\
C(\rho, U, L) & :=\frac{\rho \alpha_{2}}{|V|}\left(\mathcal{K}^{1}[L]+\left(\frac{\beta}{\lambda} \mathcal{K}^{2}[L]-|V| \mathbb{I}\right) \cdot U\right),
\end{aligned}
$$

where the macroscopic functions that appear are given by:

$$
\begin{aligned}
& \Psi^{1}(\theta):=\int_{V} \int_{V} v \psi\left(v ; v^{\prime}, \theta\right) d v d v^{\prime}, \quad \Psi^{2}(\theta):=\int_{V} \int_{V} v \otimes v^{\prime} \psi\left(v ; v^{\prime}, \theta\right) d v d v^{\prime}, \\
& \mathcal{K}^{1}[L]:=\int_{V} \int_{V} v K[\nabla L]\left(v, v^{\prime}\right) d v d v^{\prime}, \quad \mathcal{K}^{2}[L]:=\int_{V} \int_{V} v \otimes v^{\prime} K[\nabla L]\left(v, v^{\prime}\right) d v d v^{\prime} .
\end{aligned}
$$

Finally, the limiting equations for $Q$ and $L$ can be written as follows:

$$
\begin{aligned}
& \frac{\partial Q}{\partial t}=-\kappa \frac{\rho}{|V|} g(\theta) Q+\frac{k_{1} k_{-1} k_{-2} \rho}{k_{1} k_{-2} \bar{Q}+k_{-1} k_{2} L+k_{-1} k_{-2}}\left(-Q+\frac{\bar{Q}}{\left|\mathbb{S}^{N-1}\right|}\right), \\
& \frac{\partial L}{\partial t}=\kappa \frac{\rho}{|V|} \int_{\mathbb{S}^{N-1}} g(\theta) Q(\theta) d \theta-r_{L} L+\Delta_{x} L
\end{aligned}
$$

where $g(\theta)=\int_{V}\left(1-\left|\frac{\theta \cdot v}{|v|}\right|\right) d v$.

We remark here that this obtained fluid-type macroscopic model (15) contains an equation, the third one, which relates in an algebraic direct way (and not by a differential equation, thus reducing the usual complexity of these models) the macroscopic density of moving cells $\rho$ with the two densities of ECM compounds, $\bar{Q}$ and $L$, associated with the haptotaxis and chemotaxis phenomena. A simple first application of this fact is described in Section 3.2, where a comparison of simulations at microand macro-scales is stated.

\section{The Numerical Scheme}

This section is devoted to some numerical experiments designed to test in silico the accuracy of the model and to predict cell behavior. We were mainly interested in the study of the spreading of a tumor population through a formed living structure. Depending on the cancer type and the host tissue, this invasion can occur in either an individual or collective manner (see [24]) and can give rise to several type of patterns. In this sense, there is a huge literature of mathematical models for cancer invasion (see [44]), which denotes the complexity and heterogeneity of these phenomena. Therefore, we studied several simple situations, by using the presented model, which can reproduce many of these interactions between the moving cells and the surrounding ECM, and will try to measure the accuracy of the cells in adapting their invasion by following the orientation of the ECM fiber.

Simulations were obtained by solving the system (1)-(3)-(4), endowed with initial conditions $f_{0}$ and $Q_{0}$ for the cell population and the ECM, respectively, which will be described for each different situation. The system was set in a spatially-square domain (thus $N=2$ ), $\mathbb{S}^{1}$ for velocities 
(fixed modulus $s$ ), and $y$ supported in the set $Y$ defined on (11). We considered periodic boundary conditions in $x$ and zero-flux in the activity $y$. These sets were discretized using a rectangular grid for position and activity and choosing eight directions in $\mathbb{S}^{1}$.

The numerical solution of the system was obtained by using a splitting method. The idea behind this approach was to write, at least formally, the overall evolution operator as the sum of evolution operators for each term in the model, then to pick an appropriate scheme for each term and attach the pieces together. We refer the reader to [45] for more details on the technique of splitting for numerically solving partial differential equations.

Therefore, the system was split into the following subequations:

$$
\begin{aligned}
& \frac{\partial f_{n}}{\partial t}+v \cdot \nabla_{x} f_{n}+\nabla_{y} \cdot\left(G\left(y, \bar{Q}_{n-1}, L_{n-1}\right) f_{n}\right)=0, \\
& \frac{\partial f_{n}}{\partial t}=\mathcal{H}\left(f_{n}, Q_{n-1}\right)+\mathcal{L}\left(f_{n}\right)+\mathcal{C}\left(f_{n}, L_{n-1}\right), \\
& \frac{\partial Q_{n}}{\partial t}=-\kappa\left(\int_{\mathbb{S}^{1}} \int_{Y}\left(1-\left|\theta \cdot \frac{v}{|v|}\right|\right) f_{n} d v d y\right) Q_{n} \\
& \quad-k_{1} Q_{n} \int_{\mathbb{S}^{1}} \int_{Y}\left(R_{0}-y_{1}-y_{2}\right) f_{n} d v d y+\frac{k_{-1}}{2 \pi} \int_{\mathbb{S}^{1}} \int_{Y} y_{1} f_{n} d v d y, \\
& \frac{\partial L_{n}}{\partial t}= \kappa \int_{\mathbb{S}^{1}}\left(\int_{\mathbb{S}^{1}} \int_{Y}\left(1-\left|\theta \cdot \frac{v}{|v|}\right|\right) f_{n} d v d y\right) Q_{n} d \theta-r_{L} L_{n}+D_{L} \Delta_{x} L_{n} \\
&-k_{2} L_{n} \int_{\mathbb{S}^{1}} \int_{Y}\left(R_{0}-y_{1}-y_{2}\right) f_{n} d v d y+k_{-2} \int_{\mathbb{S}^{1}} \int_{Y} y_{2} f_{n} d v d y .
\end{aligned}
$$

The system was separated into three different blocks, namely: the transport equation, the integral operators that rules the changes of velocities, and the two equations for the ECM. The order in which these blocks are solved is a key point in the modeling (therefore, in the numerical scheme): a different order could give a different behavior of the system. Then, the system was solved in the same order as the blocks have been presented, i.e.:

1. First, we solved the transport term, related to the evolution of position and cell activity;

2. Second, the integral operators were treated, thus giving the new velocities;

3. Finally, we dealt with the equations for the ECM compounds, $Q$ and $L$.

We proceed to describe the techniques used in each step.

The first part of the splitting solved the modifications on position and activity of the cells. We split it again, obtaining two terms: the first one, related to the pure transport part of the equation, was solved using a finite volume scheme; the second term, $\partial_{t} f_{n}=\left(k_{1} y_{1} \bar{Q}_{n-1}+k_{2} y_{2} L_{n-1}\right) f_{n}$, obtained from the divergence of the activity, was in fact an ODE in time, where the other variables acted as parameters, so it was directly solved using a first order Euler method to go forward in time (i.e., explicit Euler).

In the second step of the splitting, for each of the three different integral operators, we had to determine the interaction kernels. In the numerical schemes for kinetic equations, this is equivalent to building a certain table (table of games) for each one of them, containing the different transition probabilities. These will describe how, from an initial velocity $\theta_{n-1}$ coming from the previous step of the simulation, we obtained a new exit orientation $\theta_{n}$ as a weighted sum of the candidates proposed for each operator.

The turning operator is simpler: the candidate orientation $\theta_{L}$ is given by either a uniform distribution or by a normal distribution in $\mathbb{S}^{1}$ centered on the initial orientation $\theta_{n-1}$.

Turning is weighted with chemotaxis by $\alpha_{i}$, so this will be the next to be described. For each point, we calculated the gradient of the chemoattractant, $\nabla_{x} L$. Next, given the entry velocity $\theta_{n-1}$, we calculated the direction of the vector $\left(\cos \left(\theta_{n-1}\right), \sin \left(\theta_{n-1}\right)\right)+K \nabla_{x} L$, named $\theta_{C}$. Here, $K$ was associated with the sensitivity to the signal. Then, the table of games was given by a normal distribution centered on $\theta_{C}$ with variance $\sigma_{C}$. 
The joint table was then built, where each value was the weighted sum (by $\alpha_{i}$ ) of each correspondent value.

For the haptotactic phenomena, the candidate orientation, $\theta_{H}$, was given in each position by the mean orientation of the fibers on the said position:

$$
\theta_{H}:=\operatorname{direction} \text { of } \int_{\mathbb{S}^{1}} \theta Q(t, x, \theta) d \theta .
$$

In order to take this velocity to the grid of possible velocities, and to build the table, we applied a normal distribution centered on $\theta_{H}$ with (small) variance $\sigma_{H}$.

Once we had both tables, we weighed them accordingly (giving more importance to haptotaxis), and we constructed the final table of games. To avoid big leaps in the changes of velocities, the new velocity was taken as the nearest orientation from the initial velocity, in the direction of the orientation given by the calculated game. Finally, the new orientation was chosen between the candidate velocity, previously calculated, and the velocity the cell already had, representing the "decision" of a cell to follow the dictated movement.

It remains to solve the equations for both chemical compounds, $Q$ and $L$. The equation for $Q$ is essentially an ODE in time (the dependence with $x$ and $\theta$ is parametric). To solve it, for each position $\mathrm{x}$ and orientation $\theta$, we numerically computed the three integrals that appeared on the right-hand side and used a first order Euler method to advance in time. For $L$, the first step was to compute the diffusion term. To do that, first, $L(t)$ was regularized using an implemented MATLAB function, SmoothN, to avoid problems with subsequent steps; then, we calculated $\Delta_{x} L(t)$ using the implemented function De12, which computed the discrete Laplacian. Then, the dependence of the equation with respect to the spatial variable could be considered parametrical, so we solved the equation as an ODE in time: numerical computation of the integral terms, and used the explicit Euler to obtain the next iteration.

From the nondimensionalization performed in the previous section, it is worth noticing that, in fact, we had two different concentrations: one, the cell-membrane concentrations of the bounded compounds $\left(R_{0}, y_{i}\right)$, whose units were (number of whatever)/("area" units); and the concentrations of the free compounds $(\bar{Q}, L)$, with units (number of whatever)/("volume" units). Therefore, a well-designed numerical scheme must take this into consideration and modify the constants in a suitable way. We modified the cell-membrane constants, now in units of (number of whatever)/("volume"), by way of calculating the ratio between the area and the volume of a cell, and we converted the concentrations accordingly. Then,

$$
\frac{\text { integrins }}{\text { volume }}=\frac{\text { integrins }}{\text { area }} \times \frac{\text { area of a cell }}{\text { volume of a cell }},
$$

which has units of concentration in a "volume". We are in dimension $N=2$, then the "area" reads for the length unit in the cell-membrane, and the "volume" is an area. If, for example, we assume that cells have the shape of a circle of diameter $d$, the conversion factor reads:

$$
\text { Integrins per "volume" unit }=\frac{4}{d} \text { Integrins per "area" unit. }
$$

In the following, we will incorporate these computations into the numerical computations, and then, both concentrations will be equally named where there is no possibility of misunderstanding.

\subsection{Adaptation of Cells to the ECM: A Measure of Alignment}

In this subsection, we introduce some alignment functions in order to measure and understand the degradation of the ECM and the adaptation of cells to its structure. We focus our 2D simulations on three situations, as suggested in [24]: straight line-oriented fiber (following the OY axis), radially-oriented fiber, and randomly-oriented fiber. In order to measure the orientation of the fiber 
and the final adaptation of the invading cells to this tissue, we can define, for any fixed orientation $\omega \in \mathbb{S}^{1}$, the normalized operators: the fiber alignment function:

$$
F_{\omega}(t, a):=\frac{\int Q(t, x, \theta)|\theta \cdot \omega| d \theta d b}{\int Q(t, x, \theta) d \theta d b}, \quad x=a \omega^{\perp}+b \omega,
$$

which measures, at any time $t$ and any perpendicular position $a$, the mean orientation of the fiber along the straight line $a \omega^{\perp}+\langle\omega\rangle$ (note that $|\theta \cdot \omega|=1$, its maximum value, fits exactly the $\omega$-orientation). Analogously, we can define the cell alignment function:

$$
C_{\omega}(t, a):=\frac{\int f(t, x, v, y) \frac{|v \cdot \omega|}{|v|} d y d v d b}{\int \rho(t, x) d b} . \quad x=a \omega^{\perp}+b \omega,
$$

which measures, at any time $t$ and any perpendicular position $a$, the relative density of cells being on the straight line $a \omega^{\perp}+\langle\omega\rangle$ and moving with velocity on the same straight line, i.e., the ratio of cells that "follow" the orientation of the ECM fiber.

For the radial case, we can decompose the position as $x=r(\cos a, \sin a)=r \hat{x}$ and use the following radial fiber alignment function,

$$
R F(t, a):=\frac{\int Q(t, \hat{x} r, \theta)|\hat{x} \cdot \theta| d \theta d r}{\int Q(t, x, \theta) d \theta d b}
$$

in order to measure the radial orientation of the fiber, $a$ standing for the selected angle. In this case, the relative density of cells following the radial pattern can be given by:

$$
R C(t, a):=\frac{\int f(t, \hat{x} r, v, y) \frac{|v \cdot \hat{x}|}{|v|} d y d v d r}{\int \rho(t, \hat{x} r) d r}, \quad x=r(\cos a, \sin a)=r \hat{x} .
$$

\subsection{Comparison between the Behavior of Activity $\rho W$ and Its Limiting Counterpart $\rho_{0} W_{0}$}

When we obtained the macroscopic limiting system in the previous section, we defined the activity moment of the solution $\rho W$, which can be interpreted as the two-component vector of the mean concentration on the cells of the two cell-membrane compounds related to the binding with the chemicals of the ECM. The evolution of this quantity can be related to the modifications on the ECM, and the movement of fibers and $L$ due to being carried by the cells.

We checked that, in the limit (15), $\rho W$ could be obtained from a linear system of equations that yields the following explicit expression (related to the equilibrium state of the cell-membrane reactions):

$$
\rho_{0} W_{0}=\frac{\rho}{k_{1} k_{-2} \bar{Q}+k_{-1} k_{2} L+k_{-1} k_{-2}}\left(\begin{array}{c}
k_{1} k_{-2} \bar{Q} \\
k_{-1} k_{2} L
\end{array}\right) .
$$

Then, we can compute $\rho W$, both via:

$$
\rho W:=\int y f(t, x, v, y) d y d v
$$

and the previous quantity. The comparison between both quantities can measure how far the different cell-membrane reactions are from the equilibrium state. 
Now, we will describe each simulation, giving the initial data for the cell population $f_{0}$ and the ECM fibers $Q_{0}$. Furthermore, each numerical experiment assumed that initially $L=0$, which represents the fact that there was no chemoattractant in the environment, and it only appeared because of the degradation of the fibers $Q$. For each simulation, we will explain its motivation and the expected behavior, and we will interpret the results. The constants used in the numerical scheme are given in Table 1.

Table 1. Constants used in the numerical scheme.

\begin{tabular}{llll}
\hline Symbol & Description of the Constant & Value & Reference \\
\hline$R_{0}$ & $\begin{array}{l}\text { Maximum concentration of integrins on the } \\
\text { cell membrane }\end{array}$ & 1000 integrins $/ \mu \mathrm{m}^{2}$ & {$[10,46]$} \\
\hline$k_{i}$ & $\begin{array}{l}\text { Binding rate of the integrins with the } \\
\text { chemical compounds }\end{array}$ & $\begin{array}{l}1.5-0.34 \mathrm{~s}^{-1}, \\
1.4-2.3 \times 10^{-4} \mu \mathrm{m}^{2} / \mathrm{s}\end{array}$ & {$[10,47,48]$} \\
\hline$k_{-i}$ & $\begin{array}{l}\text { Unbinding rate of the integrins with the } \\
\text { chemical compounds }\end{array}$ & $0.1-3.4 \mathrm{~s}^{-1}, 2.42-0.6 \mathrm{~s}^{-1}$ & {$[10,47,48]$} \\
\hline$\alpha_{1}$ & $\begin{array}{l}\text { Relative influence of the random turning in } \\
\text { the cellular movement }\end{array}$ & $1 / 27$ & {$[9]$} \\
\hline$\alpha_{2}$ & $\begin{array}{l}\text { Relative influence of the chemotaxis in the } \\
\text { cellular movement }\end{array}$ & $26 / 27$ & {$[9]$} \\
\hline$R_{C}$ & Typical size of cells & $20-200 \mu \mathrm{m}$ & {$[2]$} \\
\hline$s$ & Typical migration speed for the cells & $0.1-1.0 \mu \mathrm{m} / \mathrm{min}$ & {$[2]$} \\
\hline$r_{L}$ & Degradation rate of the chemoattractant & $5 \times 10^{-3} \mathrm{~mol} / \mathrm{s}$ & {$[49]$} \\
\hline$D_{L}$ & Diffusion coefficient of the chemical $L$ & $8 \times 10^{-6} \mathrm{~cm} / \mathrm{s}$ & {$[49]$} \\
\hline
\end{tabular}

\subsection{First Example: Fully-Oriented ECM}

We start with the first proposed scenario: initially, we had a fully-oriented ECM, in the direction of the $\mathrm{OX}_{2}$ axis, distributed following parallel (regularized) strips in said direction, creating "pathways" of oriented fiber. Therefore, at time $t=0$, the alignment function of the fiber with respect to the vertical direction $(0,1)$ was equal to one, $F_{(0,1)}(0, a)=1$ (at least where there was some fiber), and zero in any other direction, as shown in Figure 1.
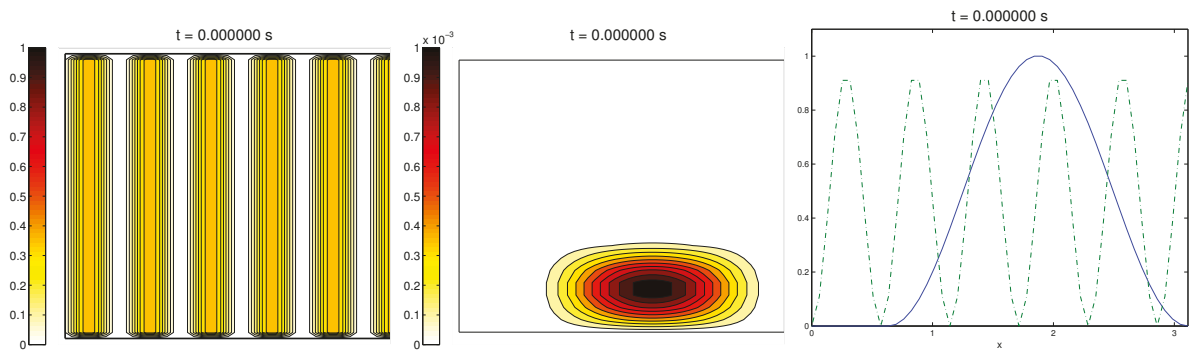

Figure 1. First example: initial conditions for the ECM fibers (oriented in the direction $(0,1)$ ), cell population, and the alignment functions $C_{(0,1)}$ (dotted) and $F_{(0,1)}$ (dashed).

For the cell population, we aimed to represent the cell invasion on a living tissue (tumoral invasion) or the creation of new structures following previously-established signals and pathways (growth of neural networks). Thus, we chose an initial cell population concentrated near the border $\{y=0\}$ and regularized, with velocities $\left(v_{1}, v_{2}\right)$ randomly chosen such that $v_{2} \geq 0$, and initial activities $y_{1}$ and $y_{2}$ were small (around $0.1 R_{0}$ ). Then, the alignment of the cells with respect to the direction $(0,1)$ was not one on every horizontal position $a$. 
Ideally, this is better represented by a fully-concentrated population in the line $\left\{x_{2}=0\right\}$ with completely random velocities. However, some regularizations and restrictions had to be made, in order to be able to perform the numerical scheme.

In this situation, we expected that $C_{(0,1)}(t, a)$ would come closer to one as time went on, meaning the alignment of the cells with the ECM; meanwhile, $F_{(0,1)}(1, a)$ should be kept close to one, as any change on the relative orientation should arise from new $Q$ incorporated by means of the cell-membrane reactions. Furthermore, the cells should move upwards, more likely following the stripes of oriented fibers, and chemical $L$ should appear from the degradation of the ECM. Finally, we expected that the cell-membrane reactions would come closer to the equilibrium state, as determined by (16). The numerical experiments confirmed our expectations: we saw that the cell population approached the strips of $Q$ and, as they reached these strips, aligned with them, following their direction. This behavior is explicit in the joint graphic for both alignment functions (Figure 2), due to the coincidence of the peaks of $C_{(0,1)}$ and $F_{(0,1)}$. In these simulations, we did not see significant ECM degradation due to the action of cells. In conclusion, we numerically confirmed that, in this example, haptotaxis dominated chemotaxis when leading cellular migration.
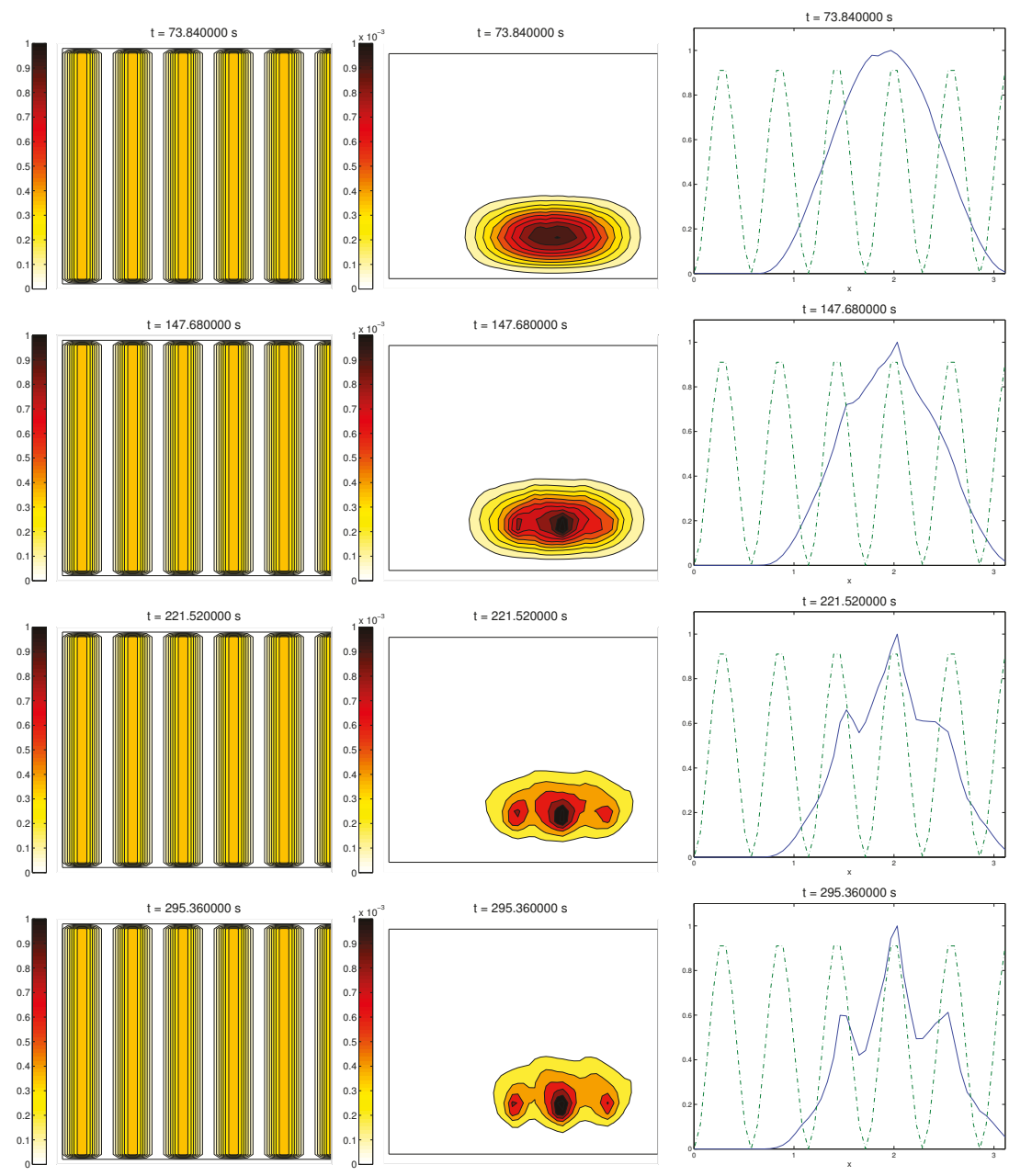

Figure 2. Cont. 

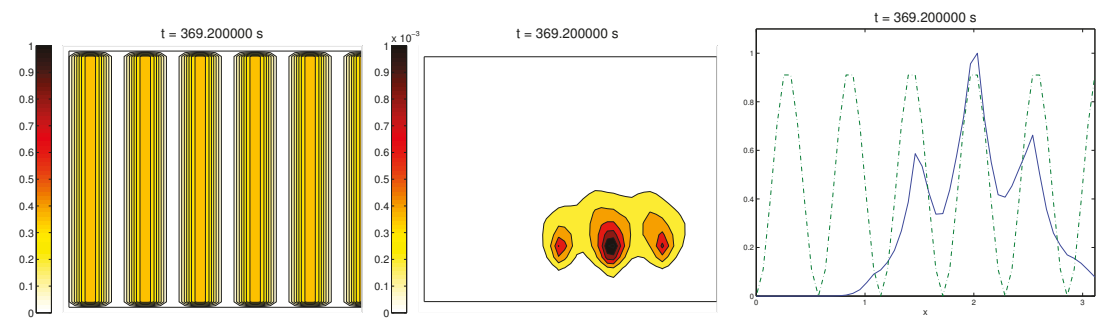

Figure 2. Graph representing the evolution of the ECM (left), cell population (center), and the alignment functions $C_{(0,1)}$ (dotted) and $F_{(0,1)}$ (dashed) for the first example.

\subsection{Second Example: Radially-Oriented ECM}

In this case study, we started with a radially-oriented distribution of proteic fibers, centered at a fixed point of the grid, which for simplicity was chosen as $(0,0)$, distributed following four straight lines equally distributed from the origin. This choice for the origin is consistent with the previous definitions of the radial orientation measures. Furthermore, the origin point would be at the center of the domain. Then, the initial radial fiber alignment function $\operatorname{RF}(0, a)$ was equal to one on the four values of the arc $a$ corresponding to the straight lines (and zero, if there was no $Q$ compound there); see Figure 3.
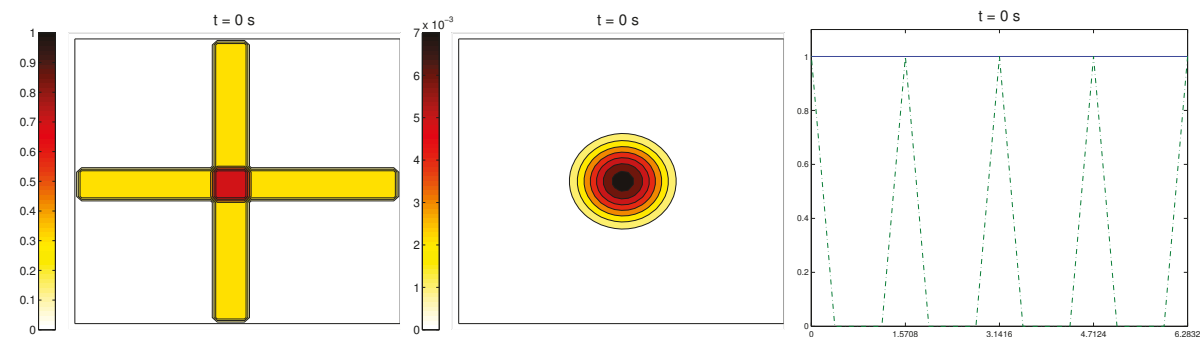

Figure 3. Second example: initial conditions for the ECM fibers, cell population, and the alignment functions $C R$ (dotted) and FR (dashed).

For the cell distribution, this situation would resemble in vitro experiments for development and invasion of a growing bacterial population. Then, $f_{0}$ was chosen as a (regularized) cut-off function of a small circular domain centered at the origin $(0,0)$, the same point from where the fiber originated. The initial velocities of cells were randomly chosen in $\mathbb{S}^{1}$. Then, the initial radial alignment operator for the cells was a random distribution, taking values between zero and one. As in the previous example, the initial activities were set small.

In this situation, we expected the same behavior as in the previous example, now with the radial alignment operators $R F$ and $R C$, instead of the fixed direction ones, $F_{\omega}$ and $C_{\omega}$, respectively. In short, $R C$ should come closer to one, meaning that cells follow the orientation ruled by the ECM, $R F$ should decrease slightly, $L$ should appear from the degradation of the matrix, and the activity should approach its equilibrium state.

Simulations partially confirmed our expectations: cells concentrate at those regions where the $Q$ compound is present and align to its orientation, following the movement along the trail, as we can see in Figure 4. We also see, in the graphic of the alignment functions $F R$ and $C R$, that the peaks of both functions are close to each other, which correspond to the influence of the other two modeled phenomena, chemotaxis and random motility. We barely see ECM degradation by means of interactions with the cell population, so these deviations must come from the random cell movement. We confirm 
again, numerically, that in this example haptotaxis dominates chemotaxis when leading the migration of cell populations.
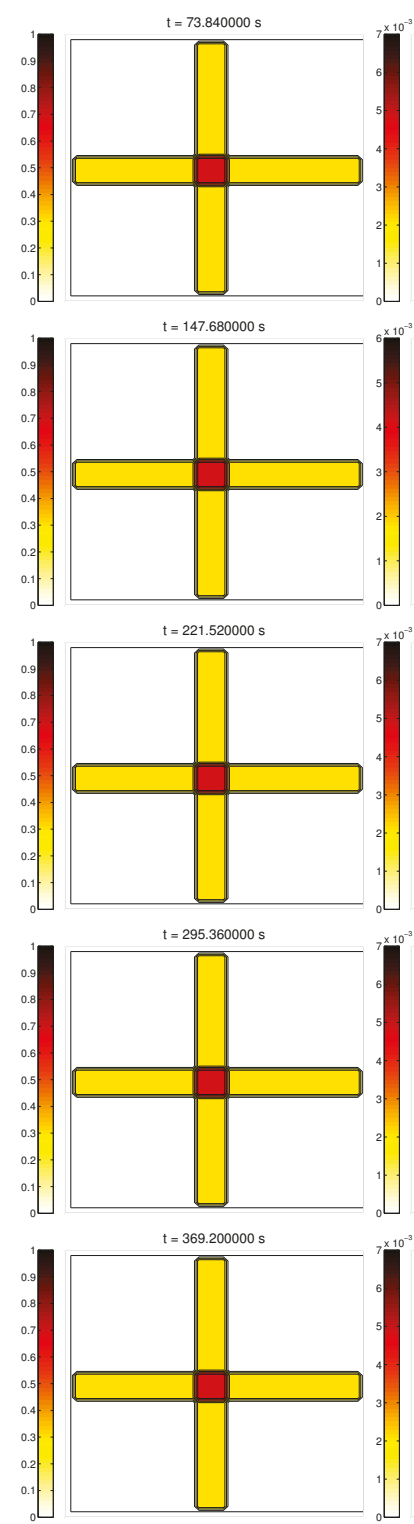

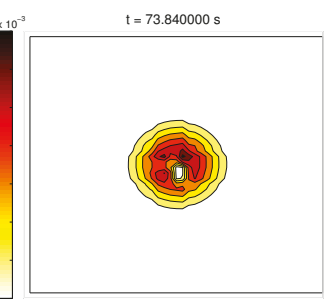

$t=147.680000 s$

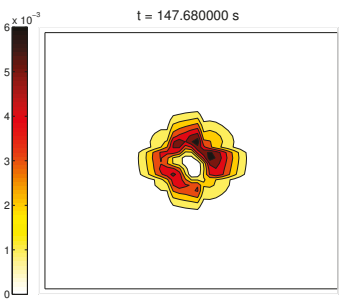

$t=221.520000 s$

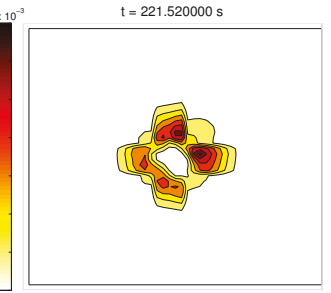

$t=295.360000 s$
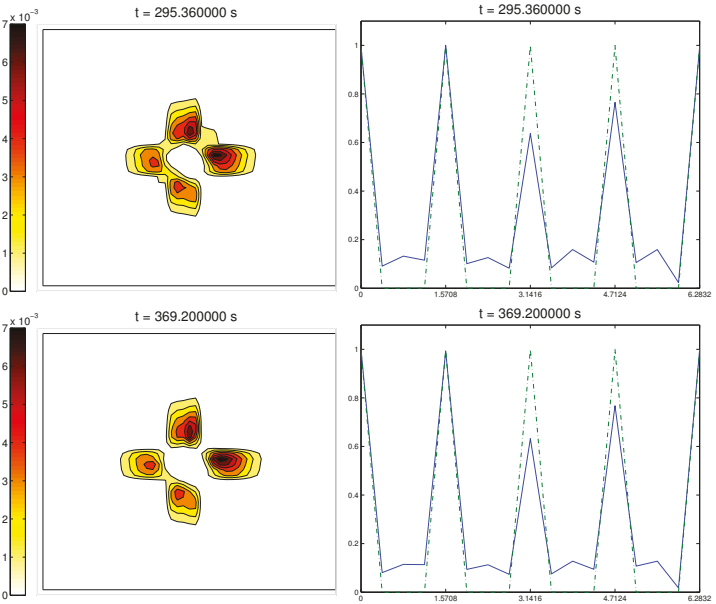

Figure 4. Graph representing the evolution of the ECM (left), cell population (center), and the alignment functions $C R_{(0,1)}$ (dotted) and $F R_{(0,1)}$ (dashed) for the second example.

\subsection{Third Example: Random ECM}

The last scenario proposed a randomly-distributed matrix, which was modified to include a certain pathway. This resembled the case of tissue development, where the ECM directs the growing of the cell population, leading to complex structures. 
For the cell distribution, we used the same initial conditions as in the first example (concentrated around $\left\{x_{2}=0\right\}$ with random velocities and small activity). Note that, in this case, measuring the alignment of the cells or the ECM with respect to a certain direction (or radial orientation), a priori, does not give any interesting information: the matrix originally did not have a preferred orientation. However, it is possible that, in subsequent steps, cells will remodel the ECM in a way that a preferential direction arises.

We expected that the cell population would concentrate in the established pathway, following this track, and few cells would remain outside the pathway. The behavior for the chemical $L$ and the limiting activity was expected to be similar to the previous examples. Furthermore, we tracked $C_{(0,1)}(t, a)$ and $F_{(0,1)}(1, a)$, to check possible remodeling on the ECM due to cell interactions or possible cell alignment caused by the creation of $L$ and the remodeled matrix. Initial conditions and results are shown in Figures 5 and 6, respectively.
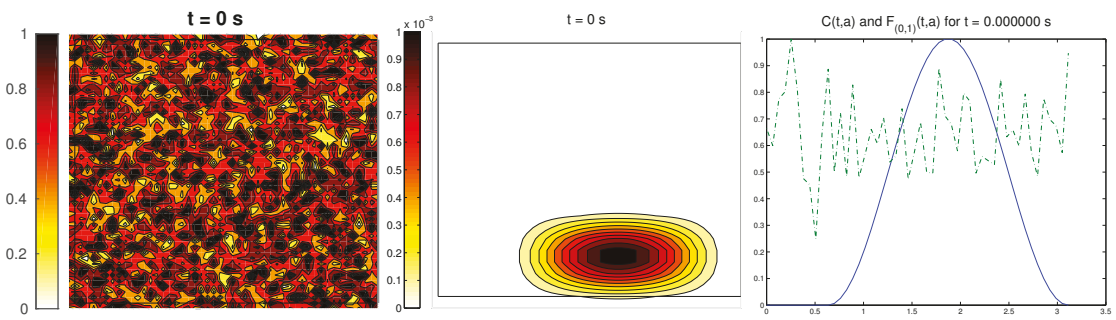

Figure 5. Third example: initial conditions for the ECM fibers (oriented in the direction $(0,1)$ ), cell population, and the alignment functions $C_{(0,1)}$ (dotted) and $F_{(0,1)}$ (dashed).
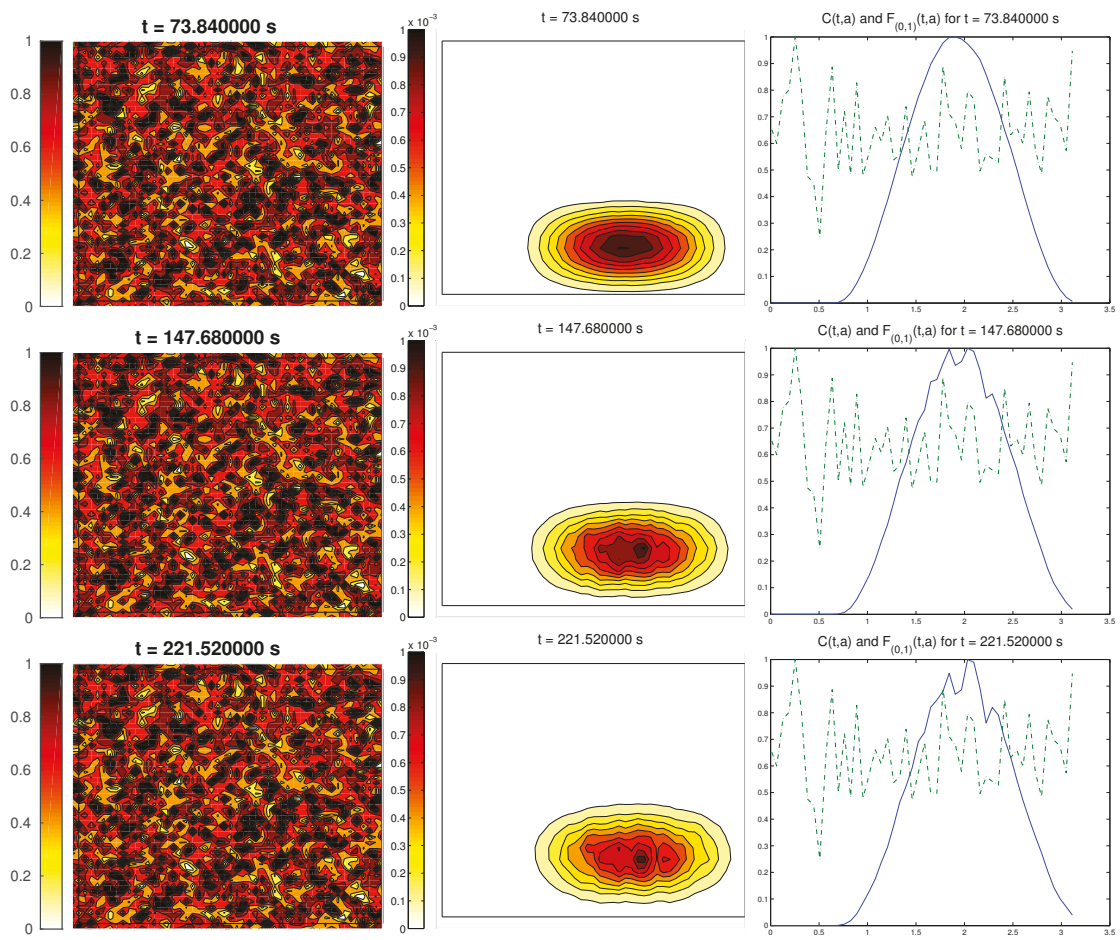

Figure 6. Cont. 

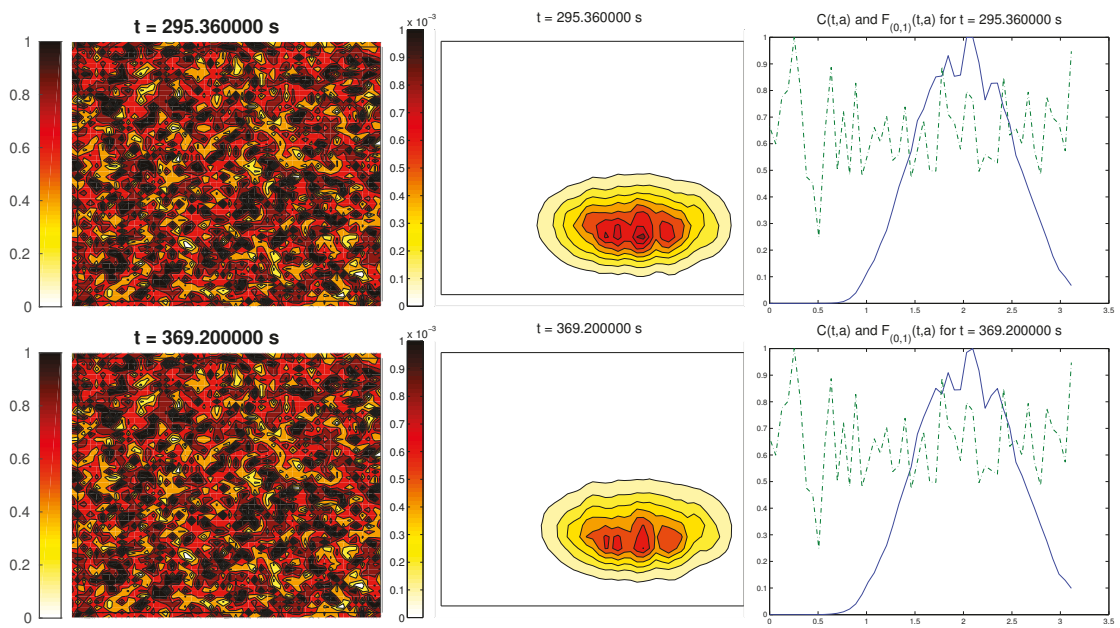

Figure 6. Graph representing the evolution of the ECM (left), cell population (center), and the alignment functions $C_{(0,1)}$ (dotted) and $F_{(0,1)}$ (dashed) for the third example.

\section{Conclusions}

In the previous sections, we presented a multiscale approach to study cell movement, caused by two important driving phenomena, namely haptotaxis and chemotaxis. The modeling was performed at the microscopic scale, and the kinetic theory of active particles allowed us to obtain a mesoscopic model. Then, a hyperbolic scaling led to the macroscopic model.

The numerical experiments aimed to explore cell behavior through three selected case studies of biological significance, as proposed in [24]. The obtained results confirmed that cells tend to follow paths and to align according to the ECM structure. In this sense, it is worth noticing the analogy with crowd dynamics [33], vehicular traffic [34], or swarming phenomena [37], where individual entities also receive some stimulus from the surrounding environment, interact among themselves, and generate certain emergent behaviors.

The alignment functionals were introduced to measure numerically the alignment of the trajectories of the cells in order to compare with the structure of the surrounding ECM. These operators could be used in the future to establish and measure similar behaviors in more complex tissue structures, as they only need the density function of the given structure.

Finally, the macroscopic fluid model obtained from the KTAP theory includes an algebraic third equation binding the densities of the two main compounds of the ECM involved in the haptotaxis and chemotaxis processes with the macroscopic density of the cell-membrane receptors, which can be used to measure, at the level of these receptors, how far the kinetic model is from the macroscopic equilibrium counterpart.

Future research perspectives include some modifications of this model in order to consider more realistic ECM structures and/or other kinds of interactions within the cells and the extracellular matrix. For instance, the incorporation of the saturation dynamics of the medium due to cellular overpopulation [27] or the inclusion of two or more subpopulations of tumor cells interacting mutually and with the surrounding tissue [28]. Furthermore, a simple kinetic system could be proposed to include biological cancer mutations involving different cell populations (quiescent, active, etc.) with different abilities, in order to obtain a more realistic measure of the alignment and the invasion profile.

Author Contributions: D.N., J.N. and L.U. equally contributed to all phases of preparation of this article.

Funding: D.K. is partially funded by Consejo Nacional de Investigaciones Científicas y Técnicas Project PIP 11220150100500 CO, Agencia Nacional de Promoción Científica y Tecnológica Project PICT 2015-1066, and 
Secretaría de Ciencia y Técnica (UNC). J.N. is partially supported by Junta de Andalucía Project P12-FQM-954 and MINECO Project RTI2018-098850-B-I00.

Conflicts of Interest: The authors declare no conflict of interest.

\section{References}

1. Ananthakrishnan, R.; Ehrlicher, A. The Forces Behind Cell Movement. Int. J. Biol. Sci. 2007, 3, $303-317$. [CrossRef] [PubMed]

2. Friedl, P.; Wolf, K. Tumour-cell invasion and migration: Diversity and escape mechanisms. Nat. Rev. Cancer 2003, 3, 362-374. [CrossRef] [PubMed]

3. Wolf, K.; Friedl, P. Molecular mechanisms of cancer cell invasion and plasticity. Br. J. Dermatol. 2006, 154 (Suppl. 1), 11-15. [CrossRef] [PubMed]

4. Keller, H.U.; Wissler, J.H.; Ploem, J. Chemotaxis is not a special case of haptotaxis. Experientia 1979, 35, 1669-1671. [CrossRef] [PubMed]

5. Elosegui-Artola, A.; Bazellières, E.; Allen, M.D.; Andreu, I.; Oria, R.; Sunyer, R.; Gomm, J.J.; Marshall, J.F.; Jones, J.L.; Trepat, X.; et al. Rigidity sensing and adaptation through regulation of integrin types. Nat. Mater. 2014, 13, 631-637. [CrossRef] [PubMed]

6. Wehrle-Haller, B. Assembly and disassembly of cell matrix adhesions. Curr. Opin. Cell Biol. 2012, 24, 569-581. [CrossRef]

7. Guo, N.H.; Krutzsch, H.C.; Negre, E.; Zabrenetzky, V.S.; Roberts, D.D. Heparin-binding peptides from the Type I repeats of Thrombospondin. J. Biol. Chem. 1992, 267, 19349-19355.

8. Guo, N.H.; Zabrenetzky, V.S; Chandrasekaran, L.; Sipes, J.M.; Lawler, J.; Krutzsch, H.C.; Roberts, D.D. Differential roles of protein Kinase C and Pertussis Toxin-sensitive G-binding proteins in modulation of melanoma cell proliferation and motility by Thrombospondin 1. Cancer Res. 1998, 58, 3154-3162.

9. Taraboletti, G.; Roberts, D.D.; Liotta, L.A. Thrombospondin-induced tumor cell migration: Haptotaxis and chemotaxis are meditated by different molecular domains. J. Cell Biol. 1987, 105, 2409-2415. [CrossRef]

10. Berry, H.; Larreta-Garde, V. Oscillatory behavior of a simple kinetic model for proteolysisis during cell invasion. Biophys. J. 1999, 77, 655-665. [CrossRef]

11. Erban, R.; Othmer, H. From signal transduction to spatial pattern formation in E. coli: A paradigm for multiscale modeling in biology. Multiscale Model. Simul. 2005, 3, 362-394. [CrossRef]

12. Patlak, C. Random walk with persistence and external bias. Bull. Math. Biophys. 1953, 15, 311-338. [CrossRef]

13. Keller, E.; Segel, L. Model for Chemotaxis. J. Theor. Biol. 1971, 30, 225-234. [CrossRef]

14. Hillen, T.; Painter, K.J. A user's guide to PDE models for chemotaxis. J. Math. Biol. 2009, 58, $183-217$. [CrossRef] [PubMed]

15. Chalub, F.A; Markovich, P.; Perthame, B.; Schmeiser, C. Kinetic models for chemotaxis and their drift-diffusion limits. Monatsh. Math. 2004, 142, 123-141. [CrossRef]

16. Othmer, H.G.; Hillen, T. The diffusion limit of transport equations II: Chemotaxis equations. SIAM J. Appl. Math. 2002, 62, 1222-1250. [CrossRef]

17. Bellomo, N.; Bellouquid, A.; Nieto, J.; Soler, J. On the asymptotic theory from microscopic to macroscopic growing tissue models: An overview with perspectives. Math. Models Methods Appl. Sci. 2012, 22, 1130001. [CrossRef]

18. Bellomo, N.; Bellouquid, A.; Tao, Y.; Winkler, M. Toward a mathematical theory of Keller-Segel models of pattern formation in biological tissues. Math. Models Methods Appl. Sci. 2015, 25, 1663-1763. [CrossRef]

19. Arias, M.; Campos, J.; Soler, J. Cross-diffusion and traveling waves in porous-media flux-saturated Keller-Segel models. Math. Models Methods Appl. Sci. 2018, 28, 2103-2129. [CrossRef]

20. Bellouquid, A.; Nieto, J.; Urrutia, L. About the kinetic description of fractional diffusion equations modeling chemotaxis. Math. Models Methods Appl. Sci. 2016, 26, 249-268. [CrossRef]

21. Bellomo, N.; Bellouquid, A.; Chouhad, N. From a multiscale derivation of nonlinear cross-diffusion models to Keller-Segel models in a Navier-Stokes fluid. Math. Model. Methods Appl. Sci. 2016, 26, 2041-2069. [CrossRef]

22. Oster, G.; Murray, J.D.; Harris, A.K. Mechanical aspects of mesenchymal morphogenesis. J. Embryol. Exp. Morphol. 1983, 78, 83-125. 
23. Mallet, D.G; Pettet, G.J. A mathematical model of integrin-mediated haptotactic cell migration. Bull. Math. Biol. 2006, 68, 231-253. [CrossRef]

24. Painter, K. Modelling cell migration strategies in the extracellular matrix. J. Math. Biol. 2009, 58, 511-543. [CrossRef]

25. Chaplain, M.A.J.; Lolas, G. Mathematical modelling of cancer cell invasion of tissue: The role of the urokinase plasminogen activation system. Math. Models Methods Appl. Sci. 2005, 15, 1685-1734. [CrossRef]

26. Kim, Y.; Jeon, H.; Othmer, H. The Role of the Tumor Microenvironment in Glioblastoma: A Mathematical Model. IEEE Trans. Biomed. Eng. 2017, 64, 519-527. [CrossRef]

27. Zhigun, A.; Surulescu, C.; Uatay, A. Global existence for a degenerate haptotaxis model of cancer invasion. Z. Angew. Math. Phys. 2016, 67, 147. [CrossRef]

28. Stinner, C.; Surulescu, C.; Uatay, A. Global existence for a go-or-grow multiscale model for tumor invasion with therapy. Math. Models Methods Appl. Sci. 2016, 11, 2163-2201. [CrossRef]

29. Bellomo, N.; Knopoff, D.; Soler, J. On the difficult interplay between life, "complexity", and mathematical sciences. Math. Models Methods Appl. Sci. 2013, 23, 1861-1913. [CrossRef]

30. Bellouquid, A.; De Angelis, E.; Knopoff, D. From the modeling of the immune hallmarks of cancer to a black swan in biology. Math. Models Methods Appl. Sci. 2013, 23, 949-978. [CrossRef]

31. Ajmone Marsan, G.; Bellomo, N.; Gibelli, L. Stochastic evolutionary differential games toward a systems theory of behavioral social dynamics. Math. Models Methods Appl. Sci. 2016, 26, 1051-1093. [CrossRef]

32. Dolfin, M.; Knopoff, D.; Leonida, L.; Patti, D. Escaping the trap of "blocking": A kinetic model linking economic development and political competition. Kinet. Relat. Models 2017, 10, 423-443. [CrossRef]

33. Bellomo, N.; Bellouquid,A.; Knopoff, D. From the micro-scale to collective crowd dynamics. Multiscale Model. Simul. 2013, 11, 943-963. [CrossRef]

34. Bellomo, N.; Bellouquid, A.; Nieto, J.; Soler, J. On the multiscale modeling of vehicular traffic: From kinetic to hydrodynamics. Discret. Cont. Dyn. Syst. Ser. B 2014, 19, 1869-1888. [CrossRef]

35. Bellomo, N.; Gibelli, L. Toward a mathematical theory of behavioral-social dynamics for pedestrian crowds. Math. Mod. Methods Appl. Sci. 2015, 25, 2417-2437. [CrossRef]

36. Bellomo, N.; Gibelli, L.; Outada, N. On the interplay between behavioral dynamics and social interactions in human crowds. Kinet. Relat. Model. 2019, 12, 397-409. [CrossRef]

37. Bellomo, N.; Ha, S.-Y. A quest toward a mathematical theory of the dynamics of swarms. Math. Mod. Methods Appl. Sci. 2017, 27, 745-770. [CrossRef]

38. Dimarco, G.; Pareschi, L. Numerical methods for kinetic equations. Acta Numer. 2014, 23, 369-520. [CrossRef]

39. Outada, N.; Vauchelet, N.; Akrid, T.; Khaladi, M. From kinetic theory of multicellular systems to hyperbolic tissue equations: Asymptotic limits and computing. Math. Mod. Methods Appl. Sci. 2016, 26, 2709-2734. [CrossRef]

40. Burini, D.; Chouhad, N. Hilbert method toward a multiscale analysis from kinetic to macroscopic models for active particles. Math. Mod. Methods Appl. Sci. 2017, 27, 1327-1353. [CrossRef]

41. Banasiak, J.; Lachowicz, M. Methods of Small Parameter in Mathematical Biology; Series: Modeling and Simulation in Science, Engineering and Technology; Birkhäuser: Boston, MA, USA, 2014.

42. Kelkel, J.; Surulescu, C. A multiscale approach to cell migration in tissue networks. Math. Mod. Methods Appl. Sci. 2012, 22, 1150017. [CrossRef]

43. Nieto, J.; Urrutia, L. A multiscale modeling of cell mobility: From kinetic to hydrodynamics. J. Math. Anal. Appl. 2016, 433, 1055-1071. [CrossRef]

44. Araujo, R.P.; McElwain, D.L.S. A history of the study of solid tumour growth: The contribution of mathematical modelling. Bull. Math. Biol. 2004, 66, 1039-1091. [CrossRef] [PubMed]

45. Holden, H.; Karlsen, K.; Lie, K.; Risebro, N. Splitting Methods for Partial Differential Equations with Rough Solutions: Analysis and MATLAB Programs; EMS Series of Lectures in Mathematics; European Mathematical Society: Zürich, Switzerland, 2010.

46. Changede, R.; Xu, X.; Margadant, F.; Sheetz, M.P.; Nascent integrin adhesions form on all matrix rigidities after integrin activation. Dev. Cell 2015, 35, 614-621. [CrossRef]

47. Welf, E.S.; Naik, U.P.; Ogunnaike, B.A. A spatial model for integrin clustering as a result of feedback between integrin activation and integrin binding. Biophys. J. 2012, 103, 1379-1389. [CrossRef] 
48. Litvinov, B.A.; Mekler, A.; Shuman, H.; Bennett, J.S.; Barsegov, V.; Weisel, J.W. Resolving two-dimensional kinetics of the integrin $\alpha I I b \beta 3$-fibrinogen interactions using binding-unbinding correlation spectroscopy. J. Biol. Chem. 2012, 287, 35275-35285. [CrossRef] [PubMed]

49. Saragosti, J.; Calvez, V.; Bournaveas, N.; Perthame, B.; Buguin, A.; Silberzan, P. Directional persistence of chemotactic bacteria in a traveling concentration wave. Proc. Natl. Acad. Sci. USA 2011, 108, 16235-16240. [CrossRef]

(c)

(C) 2019 by the authors. Licensee MDPI, Basel, Switzerland. This article is an open access article distributed under the terms and conditions of the Creative Commons Attribution (CC BY) license (http:/ / creativecommons.org/licenses/by/4.0/). 



\title{
Kinetic Model for Vehicular Traffic with Continuum Velocity and Mean Field Interactions ${ }^{\dagger}$
}

\author{
Juan Calvo $^{1}$, Juanjo Nieto ${ }^{1, *}$ and Mohamed Zagour ${ }^{2}$ \\ 1 Departamento de Matemática Aplicada and Excellence Research Unit "Modeling Nature", \\ Universidad de Granada, 18071 Granada, Spain \\ 2 Ecole Supérieure de Technologie d'Essaouira, Université Cadi Ayyad, BP. 383 Essaouira, Maroc \\ * Correspondence: jjmnieto@ugr.es \\ + To the memory of Abdelghani Bellouquid.
}

Received: 29 July 2019; Accepted: 22 August 2019; Published: 2 September 2019

check for updates

\begin{abstract}
This paper is concerned with the modeling and mathematical analysis of vehicular traffic phenomena. We adopt a kinetic theory point of view, under which the microscopic state of each vehicle is described by: (i) position, (ii) velocity and also (iii) activity, an additional varible that we use to describe the quality of the driver-vehicle micro-system. We use methods coming from game theory to describe interactions at the microscopic scale, thus constructing new models within the framework of the Kinetic Theory of Active Particles; the resulting models incorporate some of the symmetries that are commonly found in the mathematical models of the kinetic theory of gases. Short-range interactions and mean field interactions are introduced and modeled to depict velocity changes related to passing phenomena. Our main goal is twofold: (i) to use continuum-velocity variables and (ii) to introduce a non-local acceleration term modeling mean field interactions, related to, for example, the presence of tollgates or traffic highlights.
\end{abstract}

Keywords: vehicular traffic; short- and long-range interactions; kinetic theory

\section{Introduction}

The mathematical approach to vehicular traffic modeling can be developed at three different observation and representation scales, namely the microscopic, mesoscopic and macroscopic scales. Different mathematical structures correspond to each type of representation:

- microscopic scale: ordinary differential equations for the variable representing the state of each vehicle, viewed as an individual entity;

- mesoscopic scale: integro-differential equations for a probability distribution over the microscopic state of vehicles;

- $\quad$ macroscopic scale: partial differential equations for locally averaged quantities (moments of the aforementioned probability distribution); tipically density, momentum and energy are considered.

Indeed, one of the greatest difficulties of the modeling approach is the adequate choice of the representation scale. The microscopic modeling implies that we have to deal with a large number of equations and the macroscopic choice neglects the role of fluctuations and uncertainties, due the process of averaging. Note also that the last approach considers the vehicle as a single particle, instead of a system embodying both driver and mechanics; the heterogeneous behavior of the driver-vehicle micro system is not taken into account. Accordingly, the critical analysis proposed in the survey paper [1] confronts us with the fact that none of the aforesaid scale approaches is totally satisfactory; a multiscale approach is necessary to obtain a detailed description of the complex dynamics of vehicles on road. Let us comment now on some references in the literature that complement this point of 
view. In Reference [2] a hybrid model is deduced, where a detailed modeling of the dynamics of the micro-systems is implemented into a macroscopic mass conservation equation. This hybrid model is revisited in Reference [3], with the focus on the implementation of boundary conditions corresponding to the presence of tollgates, junctions or traffic highlights. This is the aim of our work but, in contrast, we will present a mesoscopic kinetic model where the velocity is still an independent variable (as for example in References [4-7]) related to the state of the driver-vehicle micro-system. We also quote here the survey on the physics and modeling of multi-particle systems [8]. The critical paper by Daganzo [9] points out some drawbacks of the driver-vehicle micro-system, where interactions can even modify the behavior of the driver -whose ability is conditioned by the local flow conditions. Reference [9] has generated various discussions and controversies documented in several works (of which we highlight Reference [10]) and also reactions to account for the aforementioned criticisms [4,5]. The approach of Reference [5] has been further developed by various papers, take for instance the proposal in Reference [6] using a discrete space variable and implementing the model over networks. Further technical developments can be found in Reference [7,11].

Our paper refers to Reference [5], where the authors proposed a kinetic model with the following main features:

1. A discrete velocity variable is used.

2. The description operates at the mesoscopic scale, in order to account for the heterogeneous behavior of the driver-vehicle micro-system.

3. The quality of the road-environment conditions is taken into account by an additional parameter. This parameter takes values between zero and one (zero for worst conditions and one for best conditions respectively) and shall play an explicit role in the description of the interactions.

Our paper is based on the kinetic theory of active particles [12] (KTAP) and draws inspiration from Reference [5]; we mention here the ability of Reference [5] to reproduce: (i) the fundamental diagrams-mean velocity and flow versus local density, (ii) clustering phenomena of vehicles with similar velocity. The analogy with social behavior KTAP models is also worth mentioning here, in particular in the field of opinion formation [13]. Heterogeneity, clustering and interactions described by evolutionary stochastic games are also key points on the modeling paradigm in this area, even if the models do not have space and velocity dependence. We extend Reference [5] by proposing a new, general mathematical structure taking into account all the possible phenomena in vehicular traffic field (short and mean-field interactions, continuous velocity and modulation of the perceived density), thus aiming at providing further developments of interest for the applications. The following modeling topics are considered: (i) interactions between vehicles accounting on perceived quantities of the vehicle flow; (ii) these interactions can be both, local and long-ranged and strongly dependent on the road conditions, (iii) dynamics under external actions (e.g., presence of tollgates). Moreover, rather than discrete velocity variables, we consider a continuous velocity distribution. These new modeling features introduced in this paper constitute what we think is a deep revision of the proposal in Reference [5].

The contents of this paper are the following: we derive a new mathematical structure in Section 2; this structure is suited to include the aforementioned features, together with those already present in Reference [5]; Section 3 shows how to derive specific models by means of inserting into the aforesaid structure various models of interactions; these models are obtained by means of a detailed phenomenological interpretation of the physical reality. Finally, Section 4 provides a brief sketch on how to construct solutions to the models introduced in Section 3.

\section{Mathematical Structures}

In this section we tackle derivation of a new, general structure, appropriate to include the specific features defined in Section 1. We do this through a sequence of subsections going from the representation of the system to the derivation of the structure. This structure is innovative with 
respect to the existing literature [5], since it includes modeling local interactions, long-range interactions and interactions with external actions as well. This structure will provide the conceptual basis for the derivation of specific models in Section 3.

\subsection{Nondimensional Representation}

Let us consider a one-dimensional flow of vehicles along a spatially periodic road of length $\ell$; the road is assumed to have a single lane. Position and velocity variables are denoted by $x$ and $v$. We introduce a limit velocity $v_{\ell}$ such that no vehicle can exceed, simply for mechanical reasons, even in the most favorable environmental conditions. We also introduce $t_{c}$, the time needed by a vehicle with velocity $v_{\ell}$ to move along the whole road, that is, $t_{c}:=\ell / v_{\ell}$. This enables us to write down dimensionless position, velocity and time variables by means of

$$
\bar{x}:=\frac{x}{\ell}, \quad \bar{v}:=\frac{v}{v_{\ell}}, \quad \bar{t}:=\frac{t}{t_{c}} .
$$

We shall drop the overlines in what follows.

We will use also dimensionless variables for macroscopic bulk quantities, as for example, the local number density $\rho=\rho(t, x)$ is defined as the actual density over $\rho_{M}$, being the latter the maximum vehicle density which corresponds to bumper-to-bumper traffic jam.

The analysis that we shall carry in this paper is based on the assumption that the state of the driver-vehicle subsystem is specified by the microscopic variables $(x, v, u) \in[0,1]^{3}$. According to the KTAP [12], $u$ is a variable that accounts for the quality of the micro-system. Namely, $u=1$ corresponds to the best quality -think of an experienced driver in a high-quality vehicle, while $u=0$ corresponds to the worst quality, that is, motion is prevented.

Following Reference [12], the subsystem driver-vehicle is regarded as an active particle, the internal variable being heterogeneously distributed over the active particles. We will use a parameter $\alpha \in[0,1]$ to measure the quality of the road including environmental conditions, that may depend on the space variable, that is, $\alpha=\alpha(x)$, in order to represent curves, local restrictions and so forth. In general, $\alpha=1$ corresponds to the best conditions and $\alpha=0$ corresponds to the worst quality that prevents motion.

We finally describe the overall state of the system by a distribution function over the states at the microscopic scale:

$$
f=f(t, x, v, u): \quad \mathbb{R}_{+} \times[0,1] \times[0,1] \times[0,1] \rightarrow \mathbb{R}_{+} .
$$

Here as usual we require $f$ to be integrable, so that $f(t, x, v, u) d x d v d u$ denotes the dimensionless distribution of vehicles which at time $t$ have position $x$, velocity $v$ and activity $u$. In particular, the local density is given by

$$
\rho(t, x)=\int_{0}^{1} \int_{0}^{1} f(t, x, v, u) d v d u .
$$

Recall that $\rho$ is normalized in term of $\rho_{M}$, which imposes a normalization on $f$.

\subsection{Interaction Domains and Perceived Quantities}

The active particle (the car-driver subsystem), has a visibility zone $\Omega_{v}=\Omega_{v}(x)=\left[x, x+\ell_{v}\right]$, where $\ell_{v}$ is the visibility length on front of the vehicle. As our road is spatially periodic, when $x+\ell_{v}>1$, the visibility zone will be $\Omega_{v}=\left[0, x+\ell_{v}-1\right] \cup[x, 1]$. This visibility length is assumed to be proportional to the quality of the environment $\alpha$ and much smaller than $\ell$. In addition, the active particle has a sensitivity zone, $\Omega_{\ell}=\left[x, x+\ell_{s}\right]$ (or $\Omega_{\ell}=\left[0, x+\ell_{s}-1\right] \cup[x, 1]$ if $x+\ell_{s}>1$ ), necessary to perceive the flow conditions in $\Omega_{\ell}$. In general $\Omega_{\ell} \subseteq \Omega_{v}$. However, we must bear in mind that the opposite case can also take place when the local conditions of the road prevent visibility. The computations in the sequel are developed under the assumption that the visibility zone includes the sensitivity zone. Note also that $\Omega_{\ell}$ can, in general, depend on $f$; this entails an additional nonlinearity. 
The driver will develop its driving strategy based on his perception of the state of the other vehicles in $\Omega_{\ell}$. Among those vehicles, the ones that are closer to the driver have a stronger influence on his strategy. To account for this, we introduce a (much) smaller domain, say $\Omega_{s}$ within which active particles are assumed to perceive an approximate estimate of the local gradients. Hence (see Reference [4]) active particles perceive a density $\rho_{p}[\rho]$ which is lower than the real one in the presence of negative gradients and higher than the real one in the presence of positive gradients. We define short-range interactions as those involving vehicles in $\Omega_{S}$ and long-range interactions as those involving vehicles mainly in $\Omega_{\ell} \backslash \Omega_{s}$.

In the approach of the KTAP, interactions are modeled after evolutionary stochastic games. We have to consider three different active particle types (driver-vehicle): (i) candidate particles, having the micro-state $\left\{x, v_{*}, u_{*}\right\}$, (ii) field particles with the state $\left\{x^{*}, v^{*}, u^{*}\right\}$ and (iii) a test particle, which is representative of the whole system. The idea is that candidate particles are localized in $x$ and can interact with field particles (considered to be localized in $\Omega_{S}$ for the case of short-range interactions and in $\Omega_{\ell}$ when dealing with long-range-that is, mean field- interactions). As a result of the interaction with the field particle, the candidate particle has a certain probability of acquiring the state of the test particle. The modeling rationale we propose in the following rests on the assumption that interactions does not modify the activity variable of both candidate and test particles.

\subsection{Mean Field Interactions}

The test vehicle is subject to interactions with those vehicles in its sensitivity zone $\Omega_{\ell}$. Both a consensus toward a common velocity (e.g., the mean speed within the visibility domain) and a clustering effect can result form this dynamics. The test vehicle becomes sensitive to these actions whenever the distance between its speed and the common velocity falls below a critical threshold.

Generally speaking, mean field interactions can be described using an individual-based acceleration term $\varphi\left(x, x^{*}, v, v^{*}, u, u^{*}\right)$. This is applied to the test vehicle $(x, v, u)$ by all the vehicles $\left(x^{*}, v^{*}, u^{*}\right)$ in its sensitivity domain $\Omega_{\ell}$. Therefore, the overall acceleration is defined as an integral accounting for the action of all vehicles in $\Omega_{\ell}$. Thus:

$$
\mathcal{F}[f](t, x, v, u)=\int_{\Lambda} \varphi\left(x, x^{*}, v, v^{*}, u, u^{*}\right) f\left(t, x^{*}, v^{*}, u^{*}\right) d x^{*} d v^{*} d u^{*},
$$

where $\Lambda:=\Omega_{\ell} \times[0,1] \times[0,1]$.

\subsection{Short-Range Interactions}

Short-range interactions involve the smaller domain $\Omega_{s}$, which is enough for a candidate particle to perceive a density $\rho_{p}$. We assume that the distribution of field particles can be approximated by the probability distribution in $x$. The description of these interactions requires to model two additional quantities:

- The encounter rate $\eta[f]$, which accounts for the number of interactions per unit time between candidate and test particles with field particles. It depends, a priori, on $t$ and $x$.

- The transition probability density $\mathcal{A}[f]\left(v_{*} \rightarrow v, v^{*}, u\right)$, which encodes the probability density that a candidate particle with velocity $v_{*}$ falls into the state of the test particle with velocity $v$ after interacting with the field particle with velocity $v^{*}$.

Now, in order to model short-range interactions we shall assume that these quantities depend on the whole distribution function $f$ (and not just on the microscopic state of the interacting particles). This dependence, which is highlighted by the square brackets, induces a nonlinearity at the microscopic scale. These interaction terms may additionally depend on the quality of the road, which is modeled by $\alpha \in[0,1]$. Finally, as a probability density, $\mathcal{A}$ is required to satisfy the condition:

$$
\mathcal{A}[f ; \alpha] \geq 0, \quad \int_{[0,1]} \mathcal{A}[f ; \alpha]\left(v_{*} \rightarrow v, v^{*}, u\right) d v=1,
$$


for all possible inputs $v_{*}, v^{*}, u$. Note that this kernel needs not be a probability density with respect to the $x$ or $u$ variables and then some integrability hypothesis should be accordingly added. Instead, we will propose an explicit expression for this term in Section 3.4; under that definition, the kernel will be a bounded function.

\subsection{Interactions with External Actions}

External actions may influence the test vehicle speed as well. Think for instance about tollgates; these enforce a maximal speed for approaching vehicles. Also, the way in which the speed can increase to the standard values after exiting the tollgate is also indicated. The effects caused by speed limits can be also described as an external action.

We model this term in the simplest way, by in using a relaxation-type term:

$$
\mathcal{T}[f](t, x, v, u)=\mu[f, x]\left(f_{e}\left(x, v_{e}(x)\right)-f(t, x, v, u)\right),
$$

where $\mu[f, x]$ represents the intensity of the action, which increases with $\rho$, while the given external density $f_{\mathcal{e}}\left(x, v_{\mathcal{e}}(x)\right)$ is suppose to adapt the velocity of the vehicles to $v_{\mathcal{e}}(x)$. Note that $\mu$ vanishes at those regions where external actions play no role.

\subsection{Assembling a Mathematical Structure}

This subsection shows how all action models that were described above can be integrated within a proper mathematical structure; this structure shall offer the conceptual basis for the derivation of different models. The structure results from a particle balance within a elementary volume in the space of microscopic states. This volume includes the phase space variables (position and velocity) together with the activity variable. The particle balance accounts for the free transport term, the transitions due to long-range interactions, the dynamics of short-range, conservative interactions (consisting in a "gain" and a "loss" term) and the trend to the velocity enforced by the external actions. At a formal level, we can write the resulting structure as follows:

$$
\partial_{t} f+v \partial_{x} f+F[f]=J[f]+\mathcal{T}[f]
$$

Here $F, J$ and $\mathcal{T}$ account for mean field interactions, short-range interactions and interactions with external actions respectively. More concretely, the structure can be written as

$$
\begin{aligned}
\partial_{t} f(t, x, v, u)+v \partial_{x} f(t, x, v, u)+\partial_{v}(\mathcal{F}[f](t, x, v, u) f(t, x, v, u)) \\
=\int_{[0,1]^{3}} \eta[f] \mathcal{A}[f ; \alpha]\left(v_{*} \rightarrow v, v^{*}, u\right) f\left(t, x, v_{*}, u\right) f\left(t, x, v^{*}, u^{*}\right) d v_{*} d v^{*} d u^{*} \\
\quad-f(t, x, v, u) \int_{[0,1]^{2}} \eta[f] f\left(t, x, v^{*}, u^{*}\right) d v^{*} d u^{*}+\mu[f, x]\left(f_{e}\left(x, v_{e}(x)\right)-f(t, x, v, u)\right) .
\end{aligned}
$$

It is worth mentioning that the nonlinear operator $J[f]$, which is also local with respect to $x$, resembles the collision kernel of Boltzmann's equation in the kinetic theory of gases; besides, the kernel $\mathcal{A}$ depends on $f$, which gives us an idea of the complexity of the mathematical structure of our approach.

\subsection{Critical Analysis}

In the authors' opinion, the following aspects of the dynamics should be taken into account when developing traffic models: the heterogeneity of the driver-vehicle subsystem, passing probabilities, aggregation dynamics for vehicles with similar velocities, the role of external actions and also the variable properties of the road-environment where the dynamics occur. We propose here a mathematical structure that is capable of including the above list of features. 
The aforesaid structure can operate as a general model derivation framework; interaction rules at the microscopic scale are fed to the global structure to obtain various models. One way to do this is by means of a phenomenological interpretation of empirical data, we quote here the book by Kerner [14] in that regard (empirical data valid in both uniform flow conditions and in transient conditions are provided; see also Reference [15]). However, we note that individual behaviors in unsteady conditions are quite different from those in steady conditions. Nevertheless, we hope that a sharp interpretation of available data will lead to models able to be validated with the information coming from new empirical data.

\section{Going from Mathematical Structures to Specific Models}

Here we develop an approach to the derivation of specific models of vehicular traffic. The procedure we outline below consists on inserting into the structure (5) models of interactions at microscopic scale. We seek a good agreement with empirical data (specifically the fundamental diagram and the emerging behaviors in unsteady flow conditions) and this will guide our descriptions of the interaction terms $\varphi, \eta, \mathcal{A}, \mu$ and $f_{e}$ characterizing the aforementioned structure.

\subsection{Modeling Accelerations}

The acceleration term $\varphi$ introduced in (2) accounts for mean field interactions. Recall that the test vehicle is subject to the action of those vehicles in front of it and within its sensitivity zone $\Omega_{\ell}$; this can induce a consensus toward a common velocity. We pose the following phenomenological interpretation of reality: the test vehicle is sensitive to these actions provided that the distance between its velocity $v$ and the observed velocity $v^{*}$ is below a certain critical threshold $d_{c}$; actually, its acceleration decays with the distance between the test and field vehicle, taking the sign of $v^{*}-v$ and being proportional to the quality of both the driver-vehicle and the road, through $u$ and $\alpha$. Then, we propose the following acceleration term:

$$
\varphi\left(x, x^{*}, v, v^{*}, u\right):=\left\{\begin{array}{lll}
\alpha u \frac{x^{*}-x}{\ell_{v}}\left(v^{*}-v\right) & \text { if } & \left|v^{*}-v\right| \leq d_{c} \\
0 & \text { if } & \left|v^{*}-v\right|>d_{c} .
\end{array}\right.
$$

Note that our definition of $\varphi$ does not depend on $u^{*}$. Whenever $x^{*} \in \Omega_{\ell}=\left[x, x+\ell_{v}\right]$ we have $0 \leq \frac{x^{*}-x}{\ell_{v}} \leq 1$ and taking $z^{*}=\frac{x^{*}-x}{\ell_{v}}$ in (2), it becomes

$$
\begin{aligned}
\mathcal{F}[f] & =\ell_{v} \int_{[0,1]^{3}} \varphi\left(x, x+\ell_{v} z^{*}, v, v^{*}, u\right) f\left(t, x+\ell_{v} z^{*}, v^{*}, u^{*}\right) d z^{*} d v^{*} d u^{*} \\
& =\alpha u \ell_{v}\left(\mathcal{F}_{1}[f](t, x)-v \mathcal{F}_{2}[f](t, x)\right),
\end{aligned}
$$

where

$$
\begin{aligned}
& \mathcal{F}_{1}[f](t, x)=\int_{0}^{1} \int_{B^{*}} \int_{0}^{1} z^{*} v^{*} f\left(t, \ell_{v} z^{*}+x, v^{*}, u^{*}\right) d z^{*} d v^{*} d u^{*}, \\
& \mathcal{F}_{2}[f](t, x)=\int_{0}^{1} \int_{B^{*}} \int_{0}^{1} z^{*} f\left(t, \ell_{v} z^{*}+x, v^{*}, u^{*}\right) d z^{*} d v^{*} d u^{*},
\end{aligned}
$$

with $B^{*}=\left\{v^{*} \in[0,1]:\left|v^{*}-v\right|<d_{c}\right\}$. In particular, the derivative of $\mathcal{F}$ with respect to $v$ yields

$$
\partial_{v}(\mathcal{F}[f])(t, x, v, u)=-\alpha u \ell_{v} \mathcal{F}_{2}[f](t, x)
$$




\subsection{Modeling the Perceived Density}

The concept of perceived density was introduced in Reference [16]. Following Reference [16], this quantity should be smaller (greater) than the real one in the presence of negative (positive) density gradients. For that aim, the following expression was introduced in Reference [4]:

$$
\rho_{p}[\rho] \equiv \rho_{p}[\rho(t, x)]:=\rho+\frac{\partial_{x} \rho}{\sqrt{1+\left(\partial_{x} \rho\right)^{2}}}\left[(1-\rho) H\left(\partial_{x} \rho\right)+\rho H\left(-\partial_{x} \rho\right)\right],
$$

where $H(\cdot)$ is the Heaviside function. Thus, the perceived density increases the value of $\rho$ to the largest admissible value $\rho=1$ in the presence of positive gradients, while in the presence of negative gradients the perceived density decreases from the actual density $\rho$ to the lowest admissible value $\rho=0$.

In our framework, which includes mean field interactions through a force term, this type of models for the perceived density can lead to incorrect results. More concretely, in previous models with discrete velocity and no acceleration, the density $\rho$ and the perceived density $\rho_{p}$ remain between zero and one (in a nondimensional representation) and this fact is essential to define the transition probability densities that are involved in the other terms. However, in our new framework with continuous velocity variable we cannot ensure a priori that the density $\rho$ will remain below one for all time; this is due to the presence of the new acceleration term.

Here we present a simplified scenario in which the above issue is fixed, keeping the spirit of formula (9) as much as possible. For that aim we introduce a controlled perceived density-which, by the way, does not involve gradients, thus producing a simpler mathematical model. If we assume that the influence of positive gradients is most relevant when: (i) the actual density is high, which tends to increase the value of the perceived density, (ii) the actual density is low, which tends to decrease the value of the perceived density, then we can propose a piecewise linear version of (9), see Figure 1 below. Actually, we propose a perceived density $\rho_{p}[\rho]$ as a Lipschitz function of $\rho$ (dashed line on the picture) which represents the effect of negative gradients for $\rho \sim 0$, the effect of positive gradients for $\rho \sim 1$ and enforces the maximum perceived density to lie between zero and one. The last property will be essential in what follows to construct the transition probability densities modeling short-range interactions.

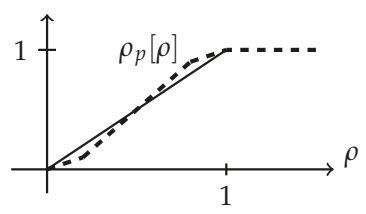

Figure 1. Cartoon showing how to construct perceived density maps (dashed line) starting from the identity map.

\subsection{Modeling the Encounter Rate}

The encounter rate $\eta[f]$ introduced in Section 2.4 describes the rate of interactions between candidate and test particles with field particles. We assume that this term increases when the local perceived density increases, starting from a minimal value $\eta_{0}$-corresponding to driving in vacuum conditions. We propose the following expression:

$$
\eta[f](t, x)=\eta_{0}\left(1+\gamma_{\eta} \rho_{p}[\rho(t, x)]\right),
$$

where $\gamma_{\eta}$ is the growth coefficient and $\rho_{p}$ is the perceived density defined in the previous paragraph. 


\subsection{Modeling Short-Range Interactions}

Let us introduce the transition probability density $\mathcal{A}[f]\left(v_{*} \rightarrow v, v^{*}, u\right)$ modeling short-range interactions. As said before, this term accounts for the probability density for a candidate particle having the state $\left\{x, v_{*}, u\right\}$ to acquire the state $\{x, v, u\}$ (state of the test particle) after interaction with the field particles with state $\left\{x, v^{*}, u^{*}\right\}$. Note that the activity variable is not modified by the interaction (which, however, modifies the speed) as hinted by the notation.

The modeling approach proposed in our paper is based on the following assumptions, which actually represent, from a continuous point of view, the velocity-discrete table of games introduced in Reference [5]:

1. Short-range interactions just modify the velocity -they do not modify the activity variable.

2. A priori, $\mathcal{A}$ should depend on the following: (i) the velocities of the interacting particles, (ii) the perceived density, (iii) the activity variable and (iv) the quality of the road. In this way, we have $\mathcal{A}=\mathcal{A}\left(v_{*} \rightarrow v, v^{*}, u, \alpha, \rho_{p}\right)$. Here the dynamics is enhanced by the product $\alpha u$ and limited by the perceived density -actually, it is totally prevented whenever $\rho_{p}=1$.

3. We assume that the candidate particle with velocity $v_{*}$, after interacting with the field particles with velocity $v^{*}$, reaches a new velocity $v$ that belongs to the interval $\left[v_{m}, v_{M}\right]$ given by

$$
\begin{aligned}
& v_{m}=\max \left\{0, \min \left\{v_{*}, v^{*}\right\}-\kappa(1-\alpha u) \rho_{p}\left(\left|v_{*}-v^{*}\right|+\exp \left(-\left|v_{*}-v^{*}\right|\right)\right)\right\}, \\
& v_{M}=\min \left\{1, \max \left\{v_{*}, v^{*}\right\}+\kappa \alpha u\left(1-\rho_{p}\right)\left(\left|v_{*}-v^{*}\right|+\exp \left(-\left|v_{*}-v^{*}\right|\right)\right)\right\} .
\end{aligned}
$$

In the above $\kappa$ is a small constant; this enables $v_{m}$ to be close to the $\min \left\{v_{*}, v^{*}\right\}$ and $v_{M}$ to be close to the $\max \left\{v_{*}, v^{*}\right\}$. Note that these choices of $v_{m}$ and $v_{M}$ guarantee the fulfillment of the condition $0<v_{m}<v_{M}<1$ even if $v_{*}=v^{*}$.

To proceed, we must consider the following two scenarios separately:

\subsubsection{Interaction with Faster Particles}

If $v_{*} \leq v^{*}$, that is, if the candidate particle detects faster field particles, it has a trend to maintain its velocity or to increase it and, in the latter case, the probability of accelerating decreases with $v-v_{m}$ where $v \in\left[v_{m}, v_{M}\right]$. We propose the following probability density,

$$
\mathcal{A}[f]\left(v_{*} \rightarrow v, v^{*}, u\right)=\left(1-\alpha u\left(1-\rho_{p}\right)\right) \frac{e^{-\frac{\left|v-v_{*}\right|^{2}}{\sigma_{1}}}}{Z\left(\sigma_{1}\right)}+\alpha u\left(1-\rho_{p}\right) \frac{\left(v_{M}-v\right)^{2}}{Z_{2}}, \quad v \in\left[v_{m}, v_{M}\right],
$$

and zero otherwise. This generalizes the table of games defined in Reference [5] in the case of discrete velocities. Here the variance $\sigma_{1}$ represents a tendency of the particle to modify its velocity under good conditions and it is given by $\sigma_{1}=\kappa \alpha u\left(1-\rho_{p}\right)$ with $\kappa \ll 1$. Moreover, $Z(\sigma)=\int_{v_{m}}^{v_{M}} e^{-\frac{\left|v-v_{*}\right|^{2}}{\sigma}} d v$ and $Z_{p}=\int_{v_{m}}^{v_{M}}\left(v_{M}-v\right)^{p} d v$ are the respective partition functions, which enforce the normalization condition $\int_{0}^{1} \mathcal{A} d v=1$. We note (see Figure 2 (left)) that this probability density has analogous nonlinear behavior as that of the table of games in Reference [5]. More precisely, in good road conditions $\alpha$ and activity $u$, the candidate particle has a tendency to accelerate, thus reaching new velocities greater than its pre-interaction velocity $v_{*}$. Quite the contrary, decreasing the value of $\alpha$ or $u$ diminishes the probability of accelerating.

\subsubsection{Interaction with Slower Particles}

When $v_{*}>v^{*}$, that is, if the candidate particle detects slower field particles, it has a tendency to decrease its velocity or to maintain it. In order to reproduce the behavior of the discrete table of 
games by Reference [5], we propose the following dichotomy function as the corresponding probability density ruling these interactions:

$$
\mathcal{A}[f]\left(v_{*} \rightarrow v, v^{*}, u\right)=\alpha u\left(1-\rho_{p}\right) \frac{e^{-\frac{\left|v-v_{*}\right|^{2}}{\sigma_{2}}}}{Z\left(\sigma_{2}\right)}+\left(1-\alpha u\left(1-\rho_{p}\right)\right) \frac{v_{M}-v}{Z_{1}}, \quad v \in\left[v_{m}, v_{M}\right] .
$$

Note that $\mathcal{A}[f]$ is set to zero for $v \notin\left[v_{m}, v_{M}\right]$. Here we have $\sigma_{2}=\kappa\left(1-\alpha u\left(1-\rho_{p}\right)\right)$; the partition functions are defined as in the previous paragraph. We remark that if $\alpha \rightarrow 0$ or $u \rightarrow 0$, the probability to decelerate is greater than the probability of keeping the current velocity. On the contrary, if $\alpha \rightarrow 1$ and $u \rightarrow 1$, the candidate particle has a tendency to maintain its velocity (see Figure 2 (right)).

Now the global transition probability density $\mathcal{A}[f]=\mathcal{A}[f]\left(v_{*} \rightarrow v, v^{*}, u\right)$ can be rewritten accordingly:

$$
\begin{aligned}
\mathcal{A}[f]\left(v_{*} \rightarrow v, v^{*}, u\right)= & {\left[H\left(v^{*}-v_{*}\right)\left((1-P) \frac{e^{-\frac{\left|v-v_{*}\right|^{2}}{\sigma_{1}}}}{Z\left(\sigma_{1}\right)}+P \frac{\left(v_{M}-v\right)^{2}}{Z_{2}}\right)\right.} \\
& \left.+\left(1-H\left(v^{*}-v_{*}\right)\right)\left(P \frac{e^{-\frac{\left|v-v_{*}\right|^{2}}{\sigma_{2}}}}{Z\left(\sigma_{2}\right)}+(1-P) \frac{v_{M}-v}{Z_{1}}\right)\right] \chi_{\left[v_{m}, v_{M}\right]}(v) .
\end{aligned}
$$

We use the shorthand notation $P=\alpha u\left(1-\rho_{p}\right)$, being $H(\cdot)$ the Heaviside function and $\chi_{B}$ the characteristic function of a set $B$.
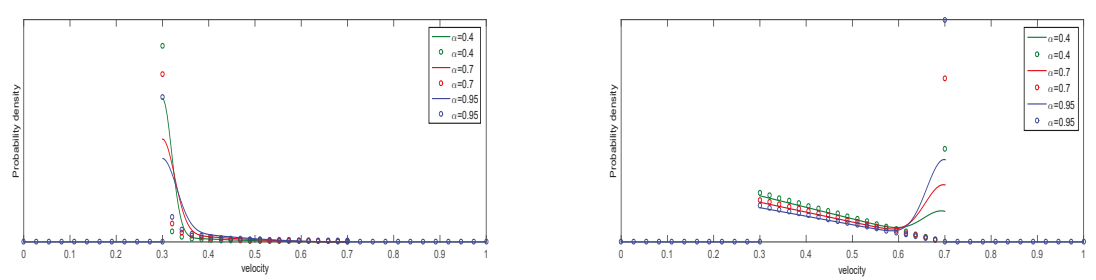

Figure 2. Comparison between discrete (from Reference [5]) and continuous transition probability densities $\mathcal{A}$. Left: interaction with faster particles. Right: interaction with slower particles.

\subsection{Modeling External Actions}

The effect of external actions is to enforce a prescribed speed, as it occurs, for example, in the presence of tollgates. This term is described as in Equation (4), where $f_{e}$ is a given function of the prescribed velocity $v_{e}=v_{e}(x)$-a step-wise function, say. Therefore, the simplicity of Equation (4) entails that only the rate $\mu$ needs to be modeled. Following the same rationale applied to the encounter rate $\eta$ in Section 3.3, the following expression can be used:

$$
\mu[f](t, x)=\mu_{0}(x)\left(1+\gamma_{\mu} \rho_{p}[\rho(t, x)]\right),
$$

where $\gamma_{\mu}$ is the growth coefficient and $\rho_{p}$ is the perceived density, whereas $\mu_{0}$ accounts for the spatial localization of the external actions.

\subsection{Parameters and Final Model}

The interaction terms to be implemented into the structure provided by Equation (5) have been modeled in a simple way along this section. The resulting model includes the following parameters, relating to diverse aspects of vehicular traffic flows:

- $\quad \alpha$ quantifies the quality of the road-weather conditions; 
- $\quad \ell_{v}$ is the length of the sensitivity zone $\Omega_{\ell}$. It depends on $\alpha$ by means of $\ell_{v}=\alpha L$;

- $\quad \eta_{0}$ is the minimal value of the encounter rate;

- $\gamma_{\eta}$ and $\gamma_{\mu}$ which stand for the growth coefficient of the encounter rate $\eta$ and the intensity of the action $\mu$, respectively.

Bearing in mind the proposed modeling of the interaction terms, we get our definitive traffic model:

$$
\begin{aligned}
\partial_{t} f(t, x, v, u)+v \partial_{x} f(t, x, v, u)+\partial_{v}(\mathcal{F}[f](t, x, v, u) f(t, x, v, u)) \\
=\quad \int_{[0,1]^{3}} \eta[f] \mathcal{A}[f]\left(v_{*} \rightarrow v\right) f\left(t, x, v_{*}, u\right) f\left(t, x, v^{*}, u^{*}\right) d v_{*} d v^{*} d u^{*} \\
\quad-f(t, x, v, u) \int_{[0,1]^{2}} \eta[f] f\left(t, x, v^{*}, u^{*}\right) d v^{*} d u^{*}+\mu[f, x]\left(f_{\mathcal{e}}\left(x, v_{\mathcal{e}}(x)\right)-f(t, x, v, u)\right) .
\end{aligned}
$$

It is worth mentioning that in this paper we have proposed a probability density (13) by drawing inspiration on the table of games proposed by Reference [5], that features discrete velocity and activity variables. Moreover, we included mean field interactions and nonlinear interactions as well (taking into account the perceived density $\rho_{p}$ ), considered as one of the paradigms of complexity. A few works proposing to model the probability density in terms of a continuous velocity variable can be found in the vehicular traffic literature, see for example, References $[7,17]$.

\section{An Iterative Method to Construct Solutions}

In this section we propose a scheme to construct a solution of (15) by means of an iterative method. We shall pose the (linear) approximating problems on the whole space $[0, \infty] \times \mathbb{R}^{3}$ in order to rely on well-known results for linear transport equations and then we will prove that this amounts to a periodic extension of the linearization of the original problem. Note that accelerations could produce, a priori, velocities out of the interval $[0,1]$, which also justifies the extension to the whole $\mathbb{R}$. With the aid of this extension we will prove in Lemma 1 below that this possibility does not actually take place. Thus, we will first consider a linearized version of model (15) on $[0, \infty] \times \mathbb{R}^{3}$ and state some properties that will enable us to construct approximate solutions.

\subsection{Linearized Transport Model}

As our starting point we consider the following transport model on $(t, x, v, u) \in[0, \infty] \times \mathbb{R}^{3}$ :

$$
\partial_{t} f+v \partial_{x} f+\partial_{v}(\mathcal{F}[h] f)=g, \quad f(0, x, v, u)=f_{0}(x, v, u) .
$$

Here $h$ and $g$ are given; these are smooth functions on $[0, \infty] \times \mathbb{R}^{3}$. We assume those to be 1 -periodic on $x$ and also to vanish for $(v, u) \notin[0,1]^{2}$. The initial data $f_{0}$ is assumed to be smooth, 1-periodic on $x$ and compactly supported on $(v, u) \in[0,1]^{2}$. The acceleration term $\mathcal{F}[h]$ is given by (7) for $(t, x, v, u) \in[0, \infty] \times \mathbb{R}^{3}$ and in this fashion it is 1-periodic in $x$. Note that our original model (15) is posed on $(t, x, v, u) \in[0, \infty] \times[0,1]^{3}$; we start by proving that, under natural hypothesis, solutions of (16) preserve the periodicity property on $x$ and the support property for $v$ and $u$.

Lemma 1 (Periodicity and support). Let $h, g$ and $f_{0}$ be smooth functions, vanishing for $(v, u) \notin[0,1]^{2}$ and 1-periodic in $x$. Then, the function $f$ verifying (16) does also vanish for $(v, u) \notin[0,1]^{2}$ and is 1-periodic in $x$, for all $t \geq 0$.

Proof. Thanks to well-known results (see References [18,19] for example), a regular solution of (16) exists, is unique and can be represented explicitly in terms of its characteristic curves, as we will see 
straightaway on formula (19). Denote these curves by $(X(s), V(s), U(s))$, they depend on an initial tuple $(t, x, v, u)$ and are determined as the solutions to

$$
\begin{aligned}
& X^{\prime}(s)=V(s), V^{\prime}(s)=\mathcal{F}[h](s, X(s), V(s), U(s)), U^{\prime}(s)=0, \quad 0 \leq s \leq t, \\
& X(t)=x, V(t)=v, U(t)=u .
\end{aligned}
$$

We will also use the notation $(X(s, x), V(s, v), U(s, u))$ whenever we need to stress the dependence on initial conditions. We remark that under our assumptions the transport field can be easily shown to be locally Lipschitz; this ensures that the associated charateristic curves given by (17) are well-defined objects in the classical sense.

Let us show that the following equality

$$
(X(s ; x)+k, V(s), u)=(X(s ; x+k), V(s), u), \forall k \in \mathbb{N}
$$

holds. Actually, we only have to observe that the acceleration term $\mathcal{F}[h]$ is 1-periodic with respect to $x$ provided $h$ is. Then we note that the initial value problems verified by both sides of equality (18) are the same. The uniquenes of this initial value problem concludes the proof of (18). Note in passing that, denoting by $J(s, t)$ the Jacobian of the change $(x, v, u) \mapsto(X(s), V(s), U(s))$ (which depends implicitly on $x$ ), we can deduce the same periodicity property (18) for $J(s, t)$; for that we just take derivatives on (18) to obtain the jacobian and use the smoothness of $h$.

Next we prove the following: if $V(t)=v \notin[0,1]$ then the $V$-component of the solution of (17) verifies that $V(s) \notin[0,1]$. It is equivalent to show the same property for the solution of the "forward" Cauchy problem (17), that is, we take $t=0$ and $s \geq 0$ and we show that $V(s ; v) \in[0,1]$ when $V(0)=v \in[0,1]$, for all $s \geq 0$. We argue by contradiction. Recall that

$$
\mathcal{F}[h]=\alpha u \ell_{v}\left(\mathcal{F}_{1}[h](t, x)-v \mathcal{F}_{2}[h](t, x)\right)
$$

and note that $\mathcal{F}_{2}[h] \geq \mathcal{F}_{1}[h]$. Assume that, for some positive time $s>0$, we have $V(s ; v)>1$ and then define $s_{1}=\inf \{s \geq 0: V(s ; v)>1\}$. Thus, $V\left(s_{1} ; v\right)=1$ and there exists some $\epsilon>0$ such that $V\left(s_{1}+\epsilon ; v\right)>1$ and $V^{\prime}\left(s_{1}+\epsilon ; v\right)>0$. We arrive to a contradiction because

$$
\begin{aligned}
0 & <V^{\prime}\left(s_{1}+\epsilon ; v\right)=\alpha u \ell_{v}\left(\mathcal{F}_{1}[h](t, X(s))-V\left(s_{1}+\epsilon ; v\right) \mathcal{F}_{2}[h](t, X(s))\right) \\
& \leq \alpha u \ell_{v}\left(\mathcal{F}_{1}[h](t, X(s))-\mathcal{F}_{2}[h](t, X(s))\right) \leq 0
\end{aligned}
$$

Analogously, arguing by contradiction we can prove that, for any positive time $s>0$, we have $V(s ; v) \geq 0$, which concludes the argument. To prove the analogous property with respect to the variable $u$ is straightforward, given that $U(s)=u$ for all $s$.

Finally, we conclude the proof of Lemma 1 by writting the solution of (16) as

$$
f(t, x, v, u)=J(0, t) f_{0}(X(0), V(0), u)+\int_{0}^{t} J(s, t) g(s, X(s), V(s), u) d s,
$$

and using the properties of $X, V$ and $U$ we just proved above.

Next lemma, where $\|\cdot\|_{L^{p}}$ stands for the norm on $L^{p}\left([0,1]^{3}\right)$, shows us some a priori estimates about the solutions, which can be found in References [18,19]; for the sake of completeness, we present here a sketch of the proof.

Lemma 2 ( $L^{p}$ estimates). Given $h, g$ and $f_{0}$ verifying the hypothesis of Lemma 1 and such that

$$
\left\|f_{0}\right\|_{L^{p}}+\|h(t, \cdot)\|_{L^{p}}+\|g(t, \cdot)\|_{L^{p}}<C<\infty
$$


for all $t \geq 0$ and $p=1, \infty$, then, (16) has a unique solution given by (19) and verifying

$$
\|f(t, \cdot)\|_{L^{p}}<C(T)<\infty
$$

for any $T>0$ and $t \in[0, T]$.

Proof. The theory of linear transport equations shows that the Jacobian satisfies $\partial_{s} J(s, t)=$ $J(s, t) \partial_{v}(\mathcal{F}[h])(s, X(s), V(s), u)$ and $J(t, t)=1$. Then, using (8), the equation for $J$ reads $\partial_{s} J(s, t)=$ $-\alpha J(s, t) u \ell_{v} \mathcal{F}_{2}[h](s, X(s))$. Upon integration,

$$
J(s, t)=\exp \left\{-\int_{t}^{s} \alpha u \ell_{v} \mathcal{F}_{2}[h](\tau, X(\tau)) d \tau\right\} .
$$

We remark that $J$ is not compactly supported in $(v, u)$; However, all the instances of $J$ in (19) are multiplied by functions that are compactly supported in $(v, u)$. Then, taking $L^{p}$ norms on (19) we obtain the desired estimate with

$$
C(T)=e^{T\|h\|_{\infty}}\left\|f_{0}\right\|_{p}+T e^{T\|h\|_{\infty}}\|g\|_{p}
$$

When $p=1$ this can be refined to $C(T)=\left\|f_{0}\right\|_{1}+\int_{0}^{t}\|g(s)\|_{1} d s$.

\subsection{The Iterative Procedure for the Full Traffic Model}

Let us now address the full model (15), posed on the whole space $[0, \infty] \times \mathbb{R}^{3}$ and with a perceived density $\rho_{p}$ given by the Lipschitz function introduced on on Section 3.2. In order to do that, let us consider, for $n \in \mathbb{N}$, the following iterative scheme:

$$
\partial_{t} f^{n+1}+v \partial_{x} f^{n+1}+\partial_{v}\left(\mathcal{F}\left[f^{n}\right] f^{n+1}\right)=g^{n}, \text { for }(t, x, v, u) \in[0, \infty] \times \mathbb{R}^{3} .
$$

This is the linear transport Equation (16) with $h=h^{n}=f^{n}$, where $g=g^{n}$ is given by:

$$
\begin{aligned}
g^{n}(t, x, v, u):= & \int_{[0,1]^{3}} \eta\left[f^{n}\right] \mathcal{A}\left[f^{n}\right]\left(v_{*} \rightarrow v\right) f^{n}\left(t, x, v_{*}, u\right) f^{n}\left(t, x, v^{*}, u^{*}\right) d v_{*} d v^{*} d u^{*} \\
& -f^{n} \int_{[0,1]^{2}} \eta\left[f^{n}\right] f^{n}\left(t, x, v^{*}, u^{*}\right) d v^{*} d u^{*}+\mu_{n}\left[f^{n}, x\right]\left(f_{e}^{n}\left(x, v_{e}(x)\right)-f^{n}(t, x, v, u)\right) .
\end{aligned}
$$

We prescribe smooth initial data $f^{n+1}(0, x, v, u)=f_{0}^{n+1}(x, u, v)$ that are 1-periodic with respect to $x$ and whose $(v, u)$-support is contained on $[0,1]^{2}$. For $n=0$, we can take for example $f^{0}(t, x, v, u)=$ $f_{0}^{0}(x, v, u)$. Here we also assume that the equilibrium $f_{e}^{n}\left(x, v_{e}(x)\right)$ is smooth, 1-periodic with respect to $x$ and such that $v_{e}(x) \in[0,1]$ for all $x$ and that

$$
\int_{[0,1]} f_{e}^{n}\left(x, v_{e}(x)\right) d x=\int_{[0,1]^{3}} f_{0}^{n}(x, v, u) d x d v d u .
$$

Moreover, we take $\mu_{n}[f, x]:=\mu_{0}^{n}(x)\left(1+\gamma_{\mu} \rho_{p}(\rho)\right)$ with $\mu_{0}^{n}$ being smooth and 1-periodic with respect to $x$.

We impose the following convergences for the given initial datum, equilibrium density and external actions intensity function:

$$
\begin{aligned}
& f_{0}^{n} \stackrel{n \rightarrow \infty}{\longrightarrow} f_{0} \text { in } L^{1}\left([0,1]^{3}\right), \quad\left\|f_{0}^{n}\right\|_{L^{\infty}\left([0,1]^{3}\right)} \leq C<\infty, \forall n \in \mathbb{N}, \\
& f_{e}^{n} \stackrel{n \rightarrow \infty}{\longrightarrow} f_{e} \text { in } L^{1}(0,1), \quad\left\|f_{e}^{n}\right\|_{L^{\infty}(0,1)} \leq C<\infty, \forall n \in \mathbb{N}, \\
& \mu_{0}^{n} \stackrel{n \rightarrow \infty}{\longrightarrow} \mu_{0} \text { in } L^{1}(0,1), \quad\left\|\mu_{0}^{n}\right\|_{L^{\infty}(0,1)} \leq C<\infty, \forall n \in \mathbb{N} .
\end{aligned}
$$


The next result gives sufficient conditions to ensure that the sequence $f^{n}$ converges to a solution of (15) with $f_{0}$ as initial data.

Theorem 1. Let $f_{0}^{n}, f_{e}^{n}$ and $\mu_{0}^{n}$ smooth sequences of initial data, equilibrium densities and external actions intensity functions respectively, all of them being 1-periodic with respect to $x$ and verifying (21). Let the sequence $f_{0}^{n}$ be supported on $[0,1]^{2}$ with respect to $(v, u)$. Then, by assuming the $L^{\infty}\left([0, \infty] ; L^{1}\left([0,1]^{3}\right)\right)$-convergence of the sequence $f^{n}$ of solutions of (20), we can ensure that the limit $f$ solves (15) in a weak sense.

Proof. We can prove by induction that $h^{n}$ and $g^{n}$ verify the hypothesis described on Lemma 1 and 2. Therefore, the sequence $f^{n}$ is 1-periodic with respect to $x$ and supported on $[0,1]^{2}$ with respect to $(v, u)$. In order prove that its limit is a solution of (15), we start from the weak formulation of (20); let a test function $\Phi \in C_{0}^{\infty}\left([0, \infty) \times(0,1)^{3}\right)$, then we have

$$
\begin{aligned}
0= & \int_{[0,1]^{3}} f_{0}^{n+1} \Phi(0, x, v, u) d x d v d u+\int_{0}^{\infty} \int_{[0,1]^{3}} f^{n+1}\left(\partial_{t} \Phi+v \partial_{x} \Phi\right) d t d x d v d u \\
& +\int_{0}^{\infty} \int_{[0,1]^{3}} f^{n+1} \mathcal{F}\left[f^{n}\right] \partial_{v} \Phi d t d x d v d u+\int_{0}^{\infty} \int_{[0,1]^{3}} \mu\left[f^{n}, x\right]\left(f_{e}^{n}-f^{n}\right) \Phi d t d x d v d u \\
& +\int_{0}^{\infty} \int_{[0,1]^{6}} \eta\left[f^{n}\right] \mathcal{A}\left[f^{n}\right] f^{n}\left(t, x, v_{*}, u\right) f^{n}\left(t, x, v^{*}, u^{*}\right) \Phi d v_{*} d v^{*} d u^{*} d t d x d v d u \\
& -\int_{0}^{\infty} \int_{[0,1]^{5}} \eta\left[f^{n}\right] f^{n}(t, x, v, u) f^{n}\left(t, x, v^{*}, u^{*}\right) \Phi d v^{*} d u^{*} d t d x d v d u
\end{aligned}
$$

Using the convergence hypothesis for $f^{n}$ and the boundness of $\varphi, \mathcal{A}\left[f^{n}\right], \eta\left[f^{n}\right]$ and $\mu\left[f^{n}, x\right]$, it is easy to deduce that each of the terms above converges. To illustrate it, let us show for example the convergence of the third term, which reads

$\int_{0}^{\infty} \int_{[0,1]^{3}} f^{n+1} \mathcal{F}\left[f^{n}\right] \partial_{v} \Phi d t d x d v d u=\int_{0}^{\infty} \int_{[0,1]^{3}} \int_{\Lambda} \partial_{v} \Phi \varphi f^{n+1}(t, x, v, u) f^{n}\left(t, x^{*}, v^{*}, u^{*}\right) d x^{*} d v^{*} d u^{*} d t d x d v d u$

We use now standard results for transport equations, for example, the results in Reference [20] to deduce the convergence of the product measure,

$$
f^{n+1}(t, x, v, u) f^{n}\left(t, x^{*}, v^{*}, u^{*}\right) \rightarrow f(t, x, v, u) f\left(t, x^{*}, v^{*}, u^{*}\right) \text { in } C\left(0, T ; \mathcal{M}\left([0,1]^{6}\right)-\text { weak }-*\right),
$$

Actually we can show that in this case the convergence takes place in $C\left(0, T ; L^{1}\left([0,1]^{6}\right)\right)$. This allows us to pass to the limit, thus obtaining

$$
\int_{0}^{\infty} \int_{[0,1]^{3}} f^{n+1} \mathcal{F}\left[f^{n}\right] \partial_{v} \Phi d t d x d v d u \rightarrow \int_{0}^{\infty} \int_{[0,1]^{3}} f \mathcal{F}[f] \partial_{v} \Phi d t d x d v d u
$$

The rest of the terms are handled in a similar way. Note here that, thanks to the boundness of the perceived density $\rho_{p}[\rho]$ and (21), the three $\mathcal{A}\left[f^{n}\right], \eta\left[f^{n}\right]$ and $\mu_{n}\left[f^{n}, x\right]$ converge pointwise and in $L^{\infty}\left([0, \infty] \times[0,1]^{3}\right)-$ weak $^{*}$ to $\mathcal{A}[f], \eta[f]$ and $\mu[f, x]$ respectively. Thus, we conclude the convergence of all the terms and then,

$$
\begin{aligned}
0= & \int_{[0,1]^{3}} f_{0} \Phi(0, x, v, u) d x d v d u+\int_{0}^{\infty} \int_{[0,1]^{3}} f\left(\partial_{t} \Phi+v \partial_{x} \Phi\right) d t d x d v d u \\
& +\int_{0}^{\infty} \int_{[0,1]^{3}} f \mathcal{F}[f] \partial_{v} \Phi d t d x d v d u+\int_{0}^{\infty} \int_{[0,1]^{3}} \mu[f, x]\left(f_{e}-f\right) \Phi d t d x d v d u \\
& +\int_{0}^{\infty} \int_{[0,1]^{6}} \eta[f] \mathcal{A}[f] f\left(t, x, v_{*}, u\right) f\left(t, x, v^{*}, u^{*}\right) \Phi d v_{*} d v^{*} d u^{*} d t d x d v d u \\
& -\int_{0}^{\infty} \int_{[0,1]^{5}} \eta[f] f(t, x, v, u) f\left(t, x, v^{*}, u^{*}\right) \Phi d v^{*} d u^{*} d t d x d v d u
\end{aligned}
$$


that is, $f$ is a weak solution of (15) with initial datum $f_{0}$.

Author Contributions: J.C., J.N. and M.Z. equally contributed to all phases of preparation of this article.

Funding: J.C. and J.N. are partially supported by Junta de Andalucía Project P12-FQM-954 and MINECO Project RTI2018-098850-B-I00. J.C. is supported by Universidad de Granada ("Plan propio de investigación, programa 9") through FEDER funds. M.Z. was supported by CNRST (Morocco), project "Modèles Mathématiques appliqués à l'environnement, à l'imagerie médicale et aux biosystèmes".

Conflicts of Interest: The authors declare no conflict of interest.

\section{References}

1. Bellomo, N.; Dogbé, C. On the modeling of traffic and crowds: A survey of models, speculations, and perspectives. SIAM Rev. 2011, 53, 409-463. [CrossRef]

2. Dolfin, M. From vehicle-driver behaviors to first order traffic flow macroscopic model. Appl. Math. Lett. 2012, 25, 2162-2167. [CrossRef]

3. Dolfin, M. Boundary conditions for first order macroscopic models of vehicular traffic in the presence of tollgates. Appl. Math. Comput. 2014, 234, 260-266. [CrossRef]

4. Bellomo, N.; Bellouquid, A.; Nieto, J.; Soler, J. On the multiscale modeling of vehicular traffic: From kinetic to hydrodynamics. Discrete Cont. Dyn. Syst. Ser. B 2014, 19, 1869-1888. [CrossRef]

5. Bellouquid, A.; De Angelis, E.; Fermo, L. Towards the modeling of vehicular traffic as a complex system: A kinetic theory approach. Math. Models Methods Appl. Sci. 2012, 22, 1140003. [CrossRef]

6. Fermo, L.; Tosin, A. A fully-discrete-state kinetic theory approach to traffic flow on road networks. Math. Models Methods Appl. Sci. 2015, 25, 423-461. [CrossRef]

7. Puppo, G.; Semplice, M.; Tosin, A.; Visconti, G. Kinetic models for traffic flow resulting in a reduced space of microscopic velocities. Kinet. Relat. Model. 2017, 10, 823-854. [CrossRef]

8. Helbing, D. Traffic and related self-driven many-particle systems. Rev. Mod. Phys. 2001, 73, 1067-1141. [CrossRef]

9. Daganzo, C.F. Requiem for second-order fluid approximations of traffic flow. Transp. Res. B 1995, 29, 277-286. [CrossRef]

10. Helbing, D.; Johansson, A. On the controversy around Daganzo's requiem and for the Aw-Rascle's resurrection of second-order traffic flow models. Eur. Phys. J. 2009, 69, 549-562. [CrossRef]

11. Puppo, G.; Semplice, M.; Tosin, A.; Visconti, G. Fundamental diagrams in traffic flow: The case of heterogeneous kinetic models. Commun. Math. Sci. 2016, 14, 643-669. [CrossRef]

12. Bellomo, N.; Knopoff, D.; Soler, J. On the difficult interplay between life, complexity and mathematical sciences. Math. Models Methods Appl. Sci. 2013, 23, 1861-1913. [CrossRef]

13. Dolfin, M.; Lachowicz, M. Modeling opinion dynamics: How the network enhances consensus. Netw. Heterog. Media 2015, 10, 877-896. [CrossRef]

14. Kerner, B.S. The Physics of Traffic; Springer: New York, NY, USA; Berlin, Germany, 2004.

15. Kerner, B.S. A theory of traffic congestion at heavy bottleneck. J. Phys. A 2008, 41, 215101. [CrossRef]

16. De Angelis, E. Nonlinear hydrodynamic models of traffic flow modelling and mathematical problems. Math. Comput. Model. 1999, 29, 83-95. [CrossRef]

17. Klar, A.; Wegener, R. A kinetic model for vehicular traffic derived from a stochastic microscopic model. Transp. Theory Stat. Phys. 1996, 25, 785-798.

18. Evans, L.C. Partial Differential Equations (2nd. ed.) (Graduate Studies in Mathematics), vol. 19; Amer. Math. Soc.: Providence, RI, USA, 2010.

19. McOwen, R.C. Partial Differential Equations: Methods and Applications; Pearson Education: NewYork, NY, USA, 2003.

20. Bellouquid, A.; Calvo, E.; Nieto, J.; Soler, J. Hyperbolic vs parabolic asymptotics in kinetic theory towards fluid dynamic models. SIAM J. Appl. Math. 2013, 73, 1327-1346. [CrossRef] 
Article

\title{
A Critical Analysis of Behavioural Crowd Dynamics-From a Modelling Strategy to Kinetic Theory Methods
}

\author{
Ahmed Elaiw ${ }^{1, *,+}$, Yusuf Al-Turki ${ }^{2,+}$ and Mohamed Alghamdi ${ }^{1,+}$ \\ 1 Department of Mathematics, Faculty of Science, King Abdulaziz University, P.O. Box 80203, Jeddah 21589, \\ Saudi Arabia \\ 2 Department of Electrical and Computer Engineering, King Abdulaziz University, Jeddah 21589, \\ Saudi Arabia \\ * Correspondence: a_m_elaiw@yahoo.com; Tel.: +966-567573631 \\ + These authors contributed equally to this work.
}

Received: 1 May 2019; Accepted: 28 June 2019; Published: 1 June 2019

check for updates

\begin{abstract}
This paper proposes a critical analysis of the literature addressed to modelling and simulations of human crowds with the aim of selecting the most appropriate scale out of the microscopic (individual based), mesoscopic (kinetic), and macroscopic (hydrodynamical) approaches. The selection is made focusing on possible applications of the model. In particular, model validation and safety problems, where validation consists of studying the ability of models to depict empirical data and observed emerging behaviors. The contents of the paper look forward to computational applications related to the flow crowds on the Jamarat bridge.
\end{abstract}

Keywords: crowd dynamics; scaling; kinetic models; safety

\section{Introduction, Motivations, and Plan of the Paper}

Our paper proposes a critical analysis of the existing literature on crowd modeling in order to select the most appropriate scale out of the microscopic (individual based), mesoscopic (kinetic), and macroscopic (hydrodynamical) scales, henceforth called micro-scale, meso-scale, and macro-scale. The selection is made focusing on possible applications of the model. Indeed, we have in mind safety problems for crowd dynamics in complex venues accounting for stress conditions and overcrowding. A strategic objective is the derivation of mathematical models suitable to depict the complexity features of the crowd and to reproduce, as far as it is possible, empirical data. Simulations are required reproduce real flow conditions and can allow to support crisis managers to improve safety conditions.

Generally, the modeling approach selects a specific scale without a preliminary study of the most appropriate one. On the other hand, the said selection is necessary to develop models appropriate to the case study under consideration. The main conceptual difficulty is induced, as mentioned in [1], by the fact that the physics, needed by the modelling and simulation of complex (living) systems, is not fully understood and mathematical models of crowd dynamics do not yet seem able to depict the whole variety of their emerging behaviors. This lack of knowledge amounts to a limited production of empirical data and makes the said selection of the scale a necessary step preliminary to the modeling approach.

The need for developing a research activity in the field of crowd dynamics is strongly motivated by the literature published in interdisciplinary journals. As an example, the review paper [2] is focused on the support to decision making towards safety of human crowds in crisis situations, see also [3,4]. Safety requires that local density remains below a critical level, which depends on the shape and the physical qualities of the venue in which the pedestrians move. More generally, crisis situations impose 
to consider high stress conditions, which can be generated by incidents $[5,6]$, as well as the role of the quality of venue on crowd movement $[7,8]$.

The presentation is mainly based on concepts which provide a general framework to support well-defined applications. Specifically, we look forward to computational applications related to the flow of crowds and safety problems on the Jamarat bridge to be treated in a forthcoming paper. In view of this application, formalizations are quite limited in this present paper. This objective requires accounting for the emotional state of the crowd, which can be heterogeneously distributed and which can have an important influence on the mechanics of the motion. The contents are as follows:

Section 2 firstly defines the main objectives of the modelling approach and of the related computational tools referred, as mentioned, to well-defined objectives. Secondly, a general modelling strategy is proposed, which is valid at all scales. Lastly, the complexity of human crowds, to be taken into account in the actual description of the collective behaviour, are reported.

Section 3 defines the general mathematical structures which are in charge of supporting the modelling approach according to the strategy defined in Section 2, where the rationale is in various steps, firstly a general structure is defined suitable for capturing the main features of the crowd and of the venue in which the crowd moves, subsequently specific models are derived inserting into the said structures specific models of the material behaviors of the crowd, and lastly a validation of models can be developed by appropriate comparisons with empirical data.

Section 4 proposes a forward look at applications, where the main objective, to be treated in a forthcoming paper [9], is the development of simulations in a venue with features analogous to those of the Jamarat bridge in Makkah.

This paper does not claim to be a survey of the existing literature which would be, however a useful general objective, but a critical analysis of a well-defined selection of articles useful to the aforementioned selection of the modelling scale. The present state of the art does not provide an exhaustive survey of crowd modeling at each scale to be followed by a critical comparisons of the achievements at each scale. Nevertheless, some surveys are available focusing separately on each scale, for instance the book [10] for the modeling at the macroscopic scale and the recent survey [11] for the modeling at the mesocopic scale. An additional bibliography is reported in Section 3.

\section{Modelling Requirements and Strategy}

Human crowds of very many individuals, as it is known, occur in venues characterized by several interconnected areas, each of them presenting different geometrical and qualitative features. An important characterization of the geometry is the presence of walls and obstacles, but also narrow corridors linking different areas, sharp changes in the path directions, and many others. We do share some definitions and specific features to characterize human crowds as they have been proposed in [12]. In the following, firstly three key questions are selected; secondly an answer, somehow inspired by [12], is given for each of them; lastly some reasonings are proposed, in italics, to enlighten a deeper vision of the problem.

- What is a crowd? Agglomeration, in the same venue, of many walkers whose collective dynamics are determined by interactions with other individuals. The assessment of a critical threshold, somewhat analogous to the Knudsen number, suitable to separate individual motion from collectively driven motion appears to be an open problem.

- Which is the most unsafe output of emotional states? The term "panic", which is occasionally criticized by experts in the field, can induce a breakdown of ordered, cooperative behaviour. Alternatively, we use the term "stress" and observe that, in many cases, stress conditions are induced by a perceived struggle for survival which drives walkers towards not safe situations rather than their survival. The concept of panic/stress should be related, as we shall see in the item below, to the concept of collective rationality/irrationality. 
- How we can define a collective intelligence? It is a collective strategy which induces an overall collective emergent behavior in a large population of walkers due to nonlinearly additive interactions which modify individual strategies and might lead to a commonly shared consensus. Consensus towards a common strategy does not imply rationality towards safe conditions, on the contrary crisis situations observe consensus towards irrational behaviors.

The answers to the three key questions will pervade our paper in the following sections consistently with the phenomenological interpretation of crowd dynamics which is delivered by the aforementioned answers and subsequent reasonings.

After these introductory reasonings, the rationale of a possible approach to modelling can be defined independently from the specific scale which is adopted towards the derivation of mathematical tools. A concise description of each step is reported below followed by the rationale and related targets, indicated in italic characters, for each step.

1. Mathematical structures to support the modelling approach: The modelling approach refers, as we shall see in the next section, to a strategic selection of one of the three scales, i.e., micro-scale (individual based), meso-scale (kinetic), and macro-scale (hydrodynamic). The selection of the scale should be developed by a preliminary analysis of the reproduction by models of the specific features of living systems. Further modelling requirements concern the validity of models and of related computational tools.

2. Derivation of a mathematical structure and modelling: All requirements reported in Item 1 should be included in a general differentials structure specialized for each scale. These structures provides the conceptual basis for the derivation of models based on a detailed analysis and modelling of walkers (pedestrians) among themselves and with walls and obstacle. In addition, the quality and main physical features of the venue, where the dynamics occur, should be taken into account.

3. Validation: Validation should be addressed to verify how far models can reproduce, quantitatively, empirical data and, qualitatively, expected emerging behaviors. The former specifically refers to the velocity diagram, representing mean velocity versus density. The latter is based on the empirical observation that collective motions exhibit a self-organization ability leading to patterns which are reproduced qualitatively, but it might be subject to large quantitative deviations for small variations of the flow conditions.

4. Computing: After validation, computational codes should be developed. As it is known [13], different mathematical structures, hence different computational tools, correspond to each scale.

5. Simulations to support crisis managers: Managers can use simulations with various specific purposes, namely "training", to support decision making. In addition, simulations can be used to improve the design of venues by comparing venues which, with equal transport ability, induce situations of minor overcrowding.

Let us now define the complexity features of crowds which have been object in [14] of sharp reasonings:

- Strategy: Individual walkers can develop strategies which take into account the geometry of the venue and the interactions with the surrounding walkers.

- Heterogeneity: The ability to express a strategy is not the same for all walkers. This strategy includes different walking targets and rules in the crowd, for instance, in evacuation it includes the possible presence of leaders.

- Learning ability: Living systems receive inputs from their environments and have the ability to learn from past experience. Therefore their strategic ability and the rules of interactions can evolve in time. Stress conditions can induce important modifications to collective behaviors.

- Nonlinear Interactions: Interactions are nonlinearly additive and involve neighbors in the sensitivity zone of each walker. In some cases, also distant walkers manage to communicate. Walkers perceive, at distance, walls and obstacles and modify their walking strategy accordingly. 
- Quality of the venue: The walking strategy, and hence the overall dynamics, is affected by the quality of the venue, where they move, for instance, environment, weather conditions, geometry, and specific features of the venue.

All aforementioned features should be taken into account, as far as it is possible, in the selection of the representation and modelling scale and subsequently in the derivation of mathematical and computational tools.

It is worth stressing that the aforementioned selection should also be related to the specific applications which are planned. Considering that the forthcoming objective is the simulation of the dynamics in the Jamarat bridge bearing in mind possible incidents, the level of emotional state is an important feature to be accounted for. In addition, heterogeneity is also important as the propagation of emotional states is extremely rapid and can, consequently, lead to disasters.

\section{On the Selection of the Mesoscopic (Kinetic) Scale}

This section deals with the selection of the kinetic theory approach out of the three classical representation and modelling scales:

- Micro-scale (individual based) corresponding to a system with finite number of degrees of freedom, where pedestrians are individually identified by their position and velocity, while rotational motion is generally neglected.

- Meso-scale (kinetic), where the micro-scale state is identified, as in individual based models, by position and velocity, but the dependent variable the probability distribution function over the micro-scale state.

- Macro-scale (hydrodynamic), where the dependent variable is defined by locally averages quantities, typically density and momentum.

The selection of the modelling scale is based on a detailed analysis on the ability of each structure to capture the complexity features of human crowds. The presentation is proposed in the following sequential steps treated in the following subsections:

(i) Assessment of the mathematical structures;

(ii) Rationale towards the validation of models;

(iii) Overview of the existing literature;

(iv) Selection of the kinetic theory approach;

(v) Critical analysis.

\subsection{Mathematical Structures}

Let us consider the general mathematical framework corresponding to the aforementioned modelling scale. This presentation mainly refer to [1], where a multiscale vision of crowd modelling is proposed. We consider a system of $N$ walkers and report, for each scale, the structures involving only the independent and dependent variables, while parameters are not here indicated as these appears in specific models. Our focus is on second order models which refer to accelerations.

Micro-scale: The state of the system is given by position $\mathbf{x}_{i}=\mathbf{x}_{i}(t)=\left(x_{i}(t), y_{i}(t)\right)$ and velocity $\mathbf{v}_{i}=\mathbf{v}_{i}(t)=\left(v_{x}^{i}(t), v_{y}^{i}(t)\right)$ of all $i$-th walkers with $i=1, \ldots, N$. The derivation of models requires the specific modellling the acceleration $\mathbf{F}_{i}(\cdot)$ impressed to each walker by the action of the other interacting walkers. The equation modelling this dynamics is as follows:

$$
\frac{d \mathbf{v}_{i}}{d t}=\mathbf{F}_{i}(\mathbf{x}, \mathbf{v}),
$$


where $\mathbf{x}=\left\{\mathbf{x}_{1}, \ldots, \mathbf{x}_{N}\right\}$, and $\mathbf{v}=\left\{\mathbf{v}_{1}, \ldots, \mathbf{v}_{N}\right\}$, while position and velocity are related, under regularity assumptions, by $\frac{d \mathbf{x}_{i}}{d t}=\mathbf{v}_{i}$. In particular the psycho-mechanical acceleration $\mathbf{F}_{i}(\cdot)$ depends on the overall geometry of the venue, as walkers visualize the walls and attempt not to collide with them.

Macro-scale: The state of the is represented by the local density $\rho=\rho(t, \mathbf{x})$ and the mean velocity $\xi=\xi(t, \mathbf{x})$. The derivation of models requires the specific modelling the acceleration $\mathcal{A}(\cdot)$ impressed the walkers in the elementary volume $\mathbf{x}$ the action the surrounding walkers. The equation modelling this dynamics is a follows:

$$
\partial_{t} \xi+\left(\xi \cdot \nabla_{\mathbf{x}}\right) \xi=\mathcal{A}(\rho, \xi),
$$

where the relation between density and mean velocity is defined by the equation of mass conservation:

$$
\partial_{t} \rho+\nabla_{\mathbf{x}} \cdot(\rho \xi)=0 .
$$

Also in this case $\mathcal{A}$ depends on the geometry of the venue as walkers, as above, visualize the walls and attempt not to collide with them.

Meso-scale: The state of system is delivered by a statistical distribution function over the micro-scale state of walkers, namely their position $\mathbf{x}$ and velocity $\mathbf{v}$ which are the micro-scale variable. As in the classical kinetic theory, macroscopic quantities are obtained by velocity weighted moments, see [15].

The mathematical structure deemed to provide the dynamics is obtained by a balance of micro-scale entities in the elementary volume of the space of micro-scale state, namely by equating the transport of $f$ to the net flow in the said micro-volume as induced by interactions:

$$
\left(\partial_{t}+\mathbf{v} \cdot \nabla_{\mathbf{x}}+\mathbf{E}(t, \mathbf{x}) \nabla_{\mathbf{v}}\right) f(t, \mathbf{x}, \mathbf{v})=(Q)(f, f)(t, \mathbf{x}, \mathbf{v}),
$$

where $\mathbf{E}$ is an external field made visual and vocal signals, while $Q$ models interactions which are nonlinear and nonlocal.

\subsection{Rationale towards Validation}

This subsection expands the brief concepts on model validation introduced in Section 2 referring to the rationale developed in [16] with focus on the kinetic theory approach. Let us firstly observe that the modelling criteria are precisely the same at all scales and specifically consists in the fulfillment of the three main abilities:

1. Capturing the complexity features of human crowds.

2. Depicting, as an emerging behavior, the velocity diagrams of crowd traffic depending on environmental conditions can determine different observable dynamics.

3. Reproduce "qualitatively" emerging behaviors which are observed in experiments.

The analysis of the literature has indicates that the following type of empirical data can be recovered:

- Velocity diagrams: These diagrams $[17,18]$ are influenced by the quality features of the venue, in addition empirical data present evidence of heterogeneous behaviors even in the case of uniform steady state uniform flow $[19,20]$.

- Walking behaviors: Empirical results have been proposed in [21-23], which can contribute to derive models.

- Development of technologies towards the individual identification of walkers $[24,25]$.

In addition, some recent contributions have proposed specific techniques towards the tuning of models, namely parameter assessment, in [26,27], where a conceptual difficulty consists in matching empirical data at the macro-scale the the dynamics at the micro-scale. 


\subsection{Overview of the Existing Literature}

The scientific and technical literature on crowd modelling and simulation is already vast and it is rapidly growing due to the impact that these studies can have on the wellbeing of the society. A critical analysis of this literature is here presented without claiming completeness, while a selection is made of contributions that appear important to the aims of our paper, colorblue namely the selection of the modelling scale.

Let us focus on the modelling at the micro-scale, where Equation (1) needs to be implemented by models of interactions between each individual with the others. Interactions with walls and trend towards the exit are generally modeled by simple rules. A model which has been well received by the scientific community is the so called social force model proposed by Helbing and coworkers [28], which has been later developed to model evacuation dynamics [12] and panic situations [5]. Still not exhaustively treated appears to be the modelling of heterogeneity, which is an important feature of crowd dynamics.

The modelling at the macro-scale is reviewed in the survey [29]. The modelling difficulty difficulty consists in understanding how the crowd selects the velocity direction and the speed by which pedestrians move. The approach, firstly proposed in [30], suggests to compute optimal trajectories by which pedestrians reach the exit. Detailed calculations are made in steady flow. More complex appears to be the problem of modelling irrational behaviors in panic situations. Additional results are reviewed in the book [10], which provides an exhaustive survey of the existing literature in the field mainly focused at the micro-scale and macro-scale.

The surveys $[13,31]$ introduce the concept of the crowds as a complex system, however, this challenging problem has not been yet exhaustively treated. In fact, heterogeneous behaviors are not taken into account by hydrodynamic models, where the averaging process suppress naturally heterogeneity. An additional problem consists is that the continuity assumption, which is typical of the hydrodynamic approach, is not consistent with physical reality of a multi-particle system where rarefied flows coexist with almost continuous flows. Paper [32] the two different descriptions suggests to weight by a parameter. However the actual modelling of this parameter remains an open problem. An interesting connection between individual based models and statistical description, in view of the kinetic theory approach is proposed in [33].

Let us now consider the modelling at the meso-scale, namely the kinetic theory approach which is far less developed than that of the micro-scale and macro-scale. Indeed, this approach has been only recently developed arguably due to the conceptual difficulties to tackle this approach. However, the methodological approach to model living systems proposed in the book [15] has contributed to this challenging aim.

The motivations to develop a kinetic theory approach were already posed in [13], however the first important contribution was given in [34] for a crowd in unbounded domains. Modelling interactions with walls and obstacles has been developed in [14], where the application has shown that two counter flows moving in a corridor, even if initially are randomly distributed, subsequently segregate into two main streams one on the left moving along one of the directions and one stream in the opposite direction. In addition two numerically minor streams move along the walls in directions opposite to that of the two main streams.

Validation of the model has been developed in [16] by showing that the the model has the ability to reproduce fundamental diagrams and observed collective emerging behaviors. Further analysis on this approach will be given in the following, referring to the selection of this scale for the modelling approach and to a specific model which will be presented later.

\subsection{Selection of the Modelling Scale}

Let us consider the selection of the most appropriate modelling scale. This choice plays a key role in the further interpretation of the remaining literature still to be examined. The analysis of the existing literature already leads to the selection of the scale which is more appropriate to achieve the objectives 
defined in Section 2. In details, the meso-scale has shown abilities to capture the complexity features of human crowd which have been presented in Section 3.1. In fact, the heterogeneous behavior of walkers can be taken into account by the dependent variable of the structure, namely a probability distribution, while the use of methods of stochastic game theory in the modelling of interactions allows to depict a broad variety of possible trends of walkers related to the interactions among themselves as well as with the geometrical and quality features venues. Different typologies of walkers from leaders to groups that need physical support to evacuate are taken into account by the partition into functional subsystems.

In principle, the said complexity features can also be inserted in models at the micro-scale. However, focusing on heterogeneity, the cost of including this specific feature is definitely high both in modelling and computing, while the local averaging needed by the derivation of hydrodynamical models eliminate local heterogeneity.

However, a technical difficulty is that that while the literature on vehicular traffic shows a valuable record of results which are documented in the excellent book by Kerner [35], which reports also about interesting emerging behaviors, as an example synchronization phenomena [36], the literature on emerging behaviors in crowd dynamics is comparatively quite limited. Nevertheless we can indicate some interesting recent contributions, in particular on the interaction between crowds and venues [37], the role of social information [38], and coarse-grained data analysis [39]. These papers support the selection developed in the following.

These reasonings support the selection of the kinetic theory approach to crowd modeling. In fact, the kinetic theory approach has the ability of capturing the afore mentioned complexity features of the crowds, of reproducing the so-called velocity diagram as an emerging behavior of interactions as shown in [16], accounting for emotional states and physical quality of the venues. These features have been enlightened as shown in [37-39], while even in the case of the approach at the hydrodynamical level models have to tackle the problem of micro-macro interactions [40,41], which can be taken into account by the kinetic theory approach.

However, the modeling approach should bear in mind multiscale problems referring, for instance, to [32], where the authors suggest to weight by an appropriate parameter the micro-scale and macro-scale description, as well as to [42] on the micro-macro derivation, and to the multiscale vision proposed in [1], where it is shown how the derivation of models at each scale is derived according to the same principles and using the same parameters.

\section{Towards Modelling Perspectives and Applications}

This final section looks ahead to research perspectives and applications. In more detail, the following topics are treated:

1. Computational tools;

2. Analytic problems;

3. Support to crisis managing.

These topics are treated in the next subsections focusing on the contribution that the kinetic theory approach can give to tackle them.

\subsection{Computational Tools}

As it is known, simulations related to classical models of the kinetic theory have been developed, since the pioneer paper by Bird [43] by Monte Carlo particle methods see also [44-47] and many others. Models of human crowds introduce additional difficulties related to the interactions which do not satisfy conservation rules besides preservation of the number of particles. However, these difficulties have been tackled in $[14,16]$, where interesting simulations, for instance on the counter-flows in long corridors, have also been proposed towards model validation. 
Interactions between pedestrians consistent with the specific features of human crowds have been taken into account in the simulations developed in $[14,16]$. Further improvements have been produced in [48] to account for the propagation of stress conditions which can be modeled by complex learning collective learning processes [49] and contagion dynamics [50].

The computational cost, in terms of computing time can be reduced if the model is simplified, for instance by discrete velocity directions, classical deterministic methods can be applied, for instance splitting methods for nonlinear transport equations [51] or finite differences [34,52]. The use of discrete models both for velocity and space has been introduced in [53] in the simpler case of vehicular traffic, where space is made discrete simply by tracts. Crowd dynamics requires a discrete areas made by triangles, while transport dynamics can be computed analogously to [53]. In parallel, developments of discrete models can contribute to modeling junctions in networks [54,55].

\subsection{Analytic Problems}

Mathematical models of crowd dynamics have an impact also on the development of the research activity on fundamental aspects of mathematical research. Let us briefly mention some of these developments. In fact the two problems presented in the following refer, respectively, on the solutions of mathematical problems and on the micro-macro of hydrodynamical models. In both cases, we indicate as possible research perspectives some open problems.

The initial value problem for kinetic crowd dynamic models has been studied in [34] for a model in the whole space. The main achievement has been the proof of existence and regularity in the large of the solutions to the initial value problem with physical bounds on the density. Namely, the proof must show that the density is bounded by the maximum physical value corresponding to full packing of individuals (as they have a dimension, that is, they are not points). On the other hand, the initial-boundary value problem for a crowd in domains which include walls is still waiting for a solution.

An additional problem is the derivation of hydrodynamical models by asymptotic perturbation methods developed by letting to zero the distance between walking individuals. Analytic results have been obtained in [42] still for a crowd in the whole space, while imposing boundary conditions creates conceptual difficulties which have not found yet a mathematical response even in the relatively simpler case of one dimensional flows [56]. The approach can be developed by expanding the probability distribution function in terms of a small parameter obtained by writing the model in dimensionless form and by letting to zero the distance between individuals. Then hydrodynamical equations are obtained by averaging terms with the same power of the aforementioned parameter, see $[57,58]$. A key problem consists in deriving hyperbolic models accounting for finite propagation speed, namely by transferring to crowd models the conceptual approach developed for swarms [59] and cellular dynamics.

\subsection{Safety Problems to Support to Crisis Managers}

Let us now analyze the literature focused on crisis management, namely on the support to the decision process of crisis managers when crisis conditions appear, thus creating overcrowded flows. In these situations, the support to the crisis manager is needed to select the most appropriate strategy to achieve safety.

The onsets of crisis situations are generally not predictable, however some concomitant causes can generate them. For instance, the quality and geometry of the venues where walkers move might enhance the propagation of panic conditions, as well as an excessive concentration of individuals in the same place. If simulations can provide accurate results, then the contribution to crisis managers can be effective to investigate possible actions suitable to reduce the aforementioned excessive concentrations. This topic will be specifically treated in the forthcoming paper [9] devoted to the development of simulations in a venue somehow related to that pilgrims find themselves in on the Jamarat bridge. More general simulations can contribute to the design of infrastructures [60] and structural safety [61]. 
An interesting research perspective consists of developing computational codes suitable to account for the propagation of emotional states from localized zones, where high stress conditions are generated by incidents. The kinetic theory approach has shown the ability to depict these specific dynamics as reported in the very recent paper [48]. The perspective to be pursued consists of developing computations in high density situations and complex geometry as they appear in the Jamarat bridge, which is the main objective of our research perspective.

Author Contributions: Conceptualization, A.E. and Y.A.-T.; methodology, A.E. and M.A.; formal analysis, M.A.; investigation, Y.A.-T.; writing original draft preparation, M.A.; writing review and editing, A.E.; project administration, A.E.

Funding: This work was funded by King Abdulaziz City for Science and Technology (KACST), Saudi Arabia, under grant No. (SP-36-200). The authors, therefore, acknowledge with thanks KACST for technical and financial support.

Conflicts of Interest: The authors declare no conflict of interest.

\section{References}

1. Aylaj, B.; Bellomo, N.; Gibelli, L.; Reali, A. On a unified multiscale vision of behavioral crowds. Math. Model. Methods Appl. Sci. 2019, 29, in press.

2. Wijermans, N.; Conrado, C.; van Steen, M.; Martella, C.; Li, J. A landscape of crowd management support: An integrative approach. Saf. Sci. 2016, 86, 142-164. [CrossRef]

3. Bellomo, N.; Clark, D.; Gibelli, L.; Townsend, P.; Vreugdenhil, B.J. Human behaviours in evacuation crowd dynamics: From modelling to big data toward crisis management. Phys. Life Rev. 2016, 18, 1-21. [CrossRef] [PubMed]

4. Pelechano, N.; Badler, N.I. Modeling crowd and trained leader behavior during building evacuation. IEEE Comput. Graph. Appl. 2006, 26, 80-86. [CrossRef] [PubMed]

5. Helbing, D.; Farkas, I.; Vicsek, T. Simulating dynamical feature of escape panic. Nature 2000, 407, 487-490. [CrossRef] [PubMed]

6. Lin, J.; Luckas, T.A. A particle swarm optimization model of emergency airplane evacuation with emotion. Netw. Hetherog. Media 2015, 10, 631-646. [CrossRef]

7. Ronchi, E.; Kuligowski, E.D.; Nilsson, D.; Peacock, R.D.; Reneke, P.A. Assessing the verification and validation of building fire evacuation models. Fire Technol. 2016, 52, 197-219. [CrossRef]

8. Ronchi, E.; Reneke, P.A.; Peacock, R.D. A conceptual fatigue-motivation model to represent pedestrian movement during stair evacuation. Appl. Math. Model. 2016, 40, 4380-4396. [CrossRef]

9. Elaiw, A.M.; Al-Turki, Y.; Alghamdi, M.A. Simulations by Kinetic Models of Human Crowds on Bridges With Internal Obstacles. Work in Progress.

10. Cristiani, E.; Piccoli, B.; Tosin, A. Multiscale Modeling of Pedestrian Dynamics; Springer: Milan, Italy, 2014.

11. Albi, G.; Bellomo, N.; Fermo, L.; Ha, S.-Y.; Kim, J.; Pareschi, L.; Poyato, D.; Soler, J. Traffic, crowds, and swarms. From kinetic theory and multiscale methods to applications and research perspectives. Math. Model. Methods Appl. Sci. 2019, 29. [CrossRef]

12. Helbing, D.; Johansson, A. Pedestrian crowd and evacuation dynamics. In Enciclopedia of Complexity and System Science; Springer: Berlin, Germany, 2009; pp. 6476-6495.

13. Bellomo, N.; Dogbè, C. On the modeling of traffic and crowds: A survey of models, speculations, and perspectives. SIAM Rev. 2011, 53, 409-463. [CrossRef]

14. Bellomo, N.; Gibelli, L. Toward a behavioral-social dynamics of pedestrian crowds. Math. Model. Methods Appl. Sci. 2015, 25, 2417-2437. [CrossRef]

15. Bellomo, N.; Bellouquid, A.; Gibelli, L.; Outada, N. A Quest Towards a Mathematical Theory of Living Systems; Birkhauser-Springer: New York, NY, USA, 2017.

16. Bellomo, N.; Gibelli, L. Behavioral crowds: Modeling and Monte Carlo simulations toward validation. Comput. Fluids 2016, 141, 13-21. [CrossRef]

17. Schadschneider, A.; Seyfried, A. Empirical results for pedestrian dynamics and their implications for Cellular Automata models. In Pedestrian Behavior-Models, Data Collection and Applications; Emerald Group Publishing: Bingley, West Yorkshire, UK, 2009; pp. 27-44. 
18. Schadschneider, A.; Seyfried, A. Empirical results for pedestrian dynamics and their implications for modeling. Netw. Heterog. Media 2011, 6, 545-560.

19. Schadschneider, A.; Chraibi, M.; Seyfried, A.; Tordeux, A.; Zhang, J. Pedestrian dynamics: From empirical results to modeling. In Crowd Dynamics Voume 1-Theory Models and Safety Problems; Gibelli, L., Bellomo, N., Eds.; Springer: Berlin, Germany, 2018; pp. 63-102.

20. Seyfried, A.; Steffen, B.; Klingsch, W.; Boltes, M. The fundamental diagram of pedestrian movement revisited. J. Stat. Mech. Theory Exp. 2006, 360, 232-238. [CrossRef]

21. Daamen, W.; Hoogedorn, S.P. Experimental research of pedestrian walking behavior. In Proceedings of the TRB Annual Meeting CD-ROM, Washington, DC, USA, 22-26 January 2006.

22. Moussaid, M.; Helbing, D.; Garnier, S.; Johanson, A.; Combe, M.; Theraulaz, G. Experimental study of the behavioral underlying mechanism underlying self-organization in human crowd. Proc. R. Soc. B Biol. Sci. 2009, 276, 2755-2762. [CrossRef] [PubMed]

23. Moussaïd, M.; Theraulaz, G. Comment les piétons marchent dans la foule. La Recherche 2011, 450, 56-59.

24. Kirchner, A.; Schadschneider, A. Simulation of evacuation processes using a bionics-inspired cellular automaton model for pedestrian dynamics. Physics A 2002, 312, 260-276. [CrossRef]

25. Roggen, D.; Wirz, M.; Tröster, G.; Helbing, D. Recognition of crowd behavior from mobile sensors with pattern analysis and graph clustering methods. Netw. Heterog. Media 2011, 6, 521-544.

26. Corbetta, A.; Mountean, A.; Vafayi, K. Parameter estimation of social forces in pedestrian dynamics models via probabilistic method. Math. Biosci. Eng. 2015, 12, 337-356.

27. Corbetta, A.; Bruno, L.; Mountean, A.; Yoschi, F. High statistics measurements of pedestrian dynamics, models via probabilistic method. Transp. Res. Proc. 2014, 2, 96-104. [CrossRef]

28. Helbing, D.; Molnár, P. Social force model for pedestrian dynamics. Phys. Rev. E 1995, 51, 4282-4286. [CrossRef]

29. Hughes, R.L. The flow of human crowds. Annu. Rev. Fluid Mech. 2003, 169-182. [CrossRef]

30. Hughes, R.L. A continuum theory for the flow of pedestrians. Transp. Res. B 2002, 36, 507-536. [CrossRef]

31. Bellomo, N.; Piccoli, B.; Tosin, A. Modeling crowd dynamics from a complex system viewpoint. Math. Model. Methods Appl. Sci. 2012, 22, 1230004. [CrossRef]

32. Cristiani, E.; Piccoli, B.; Tosin, A. Multiscale modeling of granular flows with application to crowd dynamics. Multiscale Model. Simul. 2011, 9, 155-182. [CrossRef]

33. Helbing, D. Traffic and related self-driven many-particle systems. Rev. Mod. Phys. 2001, 73, 1067-1141. [CrossRef]

34. Bellomo, N.; Bellouquid, A.; Knopoff, D. From the micro-scale to collective crowd dynamics. Multiscale Model. Simul. 2013, 11, 943-963. [CrossRef]

35. Kerner, B. The Physics of Traffic; Springer: New York, NY, USA; Heidelberg, Germany, 2004.

36. Kerner, B.; Klenov, S. A microscopic model for phase transitions in traffic flow. J. Phys. A 2002, 35, 31-43. [CrossRef]

37. Pinter-Wollman, N.; Penn, A.; Theraulaz, G.; Fiore, S.M. Interdisciplinary approaches for uncovering the impacts of architecture on collective behaviour. Philos. Trans. R. Soc. B Biol. Sci. 2018, 373, 20170232. [CrossRef]

38. Jayles, B.; Kim, H.R.; Escobedo, R.; Cezera, S.; Blanchet, A.; Kameda, T.; Sire, C.; Theraulaz, G. How social information can improve estimation accuracy in human groups. Proc. Natl. Acad. Sci. USA 2017, 114, 12620-12625. [CrossRef]

39. Motsch, S.; Moussaïd, M.; Guillot, E.G.; Moreau, M.; Pettré, J.; Theraulaz, G.; Appert-Rolland, C.; Degond, P. Modeling crowd dynamics through coarse-grained data analysis. Math. Biosci. Eng. 2018, 15, 1271-1290. [CrossRef] [PubMed]

40. Colombo, R.; Lécureux-Mercier, M. An analytical framework to describe the interactions between individuals and a continuum. J. Nonlinear Sci. 2012, 22, 39-61. [CrossRef]

41. Degond, P.; Appert-Rolland, C.; Moussaid, M.; Pettré, J.; Theraulaz, G. A hierarchy of heuristic-based models of crowd dynamics. J. Stat. Phys. 2013, 152, 1033-1068. [CrossRef]

42. Bellomo, N.; Bellouquid, A. On multiscale models of pedestrian crowds from mesoscopic to macroscopic. Commun. Math. Sci. 2015, 13, 1649-1664. [CrossRef]

43. Bird, G.A. Molecular Gas Dynamics and the Direct Simulation of Gas Flows; Oxford University Press: Oxford, UK, 1994. 
44. Aristov, V.V. Direct Methods for Solving the Boltzmann Equation and Study of Nonequilibrium Flows; Springer: New York, NY, USA, 2001.

45. Barbante, P.; Frezzotti, A.; Gibelli, L. A kinetic theory description of liquid menisci at the microscale. Kinet. Relat. Model. 2015, 8, 235-254.

46. Dimarco, G.; Pareschi, L. Numerical methods for kinetic equations. Acta Numer. 2014, 23, 369-520. [CrossRef]

47. Pareschi, L.; Toscani, G. Interacting Multiagent System. Kinetic Equations and Monte Carlo Methods; Oxford University Press: Oxford, UK, 2013.

48. Bellomo, N.; Gibelli, L.; Outada, N. On the interplay between behavioral dynamics and social interactions in human crowds. Kinet. Relat. Model. 2019, 12, 397-409. [CrossRef]

49. Burini, D.; de Lillo, S.; Gibelli, L. Stochastic differential "nonlinear" games modeling collective learning dynamics. Phys. Life Rev. 2016, 16, 123-139. [CrossRef] [PubMed]

50. Wang, L.; Short, M.; Bertozzi, A.L. Efficient numerical methods for multiscale crowd dynamics with emotional contagion. Math. Model. Methods Appl. Sci. 2017, 27, 205-230. [CrossRef]

51. Kim, D.; Quaini, A. A kinetic theory approach to model pedestrian dynamics in bounded domains with obstacles. arXiv 2019, arXiv:1901.07620v2.

52. Agnelli, J.P.; Colasuonno, F.; Knopoff, D. A kinetic theory approach to the dynamics of crowd evacuation from bounded domains. Math. Model. Methods Appl. Sci. 2015, 25, 109-129. [CrossRef]

53. Fermo, L.; Tosin, A. A fully-discrete-state kinetic theory approach to modeling vehicular traffic. SIAM J. Appl. Math. 2013, 73, 1533-1556. [CrossRef]

54. Akbarzadeh, M.; Estrada, E. Communicability geometry captures traffic flows in cities. Nat. Hum. Behav. 2018, 2, 645-652. [CrossRef]

55. Bressan, A.; Canic, S.; Garavello, M.; Herty, M.; Piccoli, B. Flows on networks: Recent results and perspectives. EMS Surv. Math. Sci. 2014, 1, 47-111. [CrossRef]

56. Bellomo, N.; Bellouquid, A.; Nieto, J.; Soler, J. On the multiscale modeling of vehicular traffic: From kinetic to hydrodynamics. Discret. Contin. Dyn. Syst. Ser. B 2014, 19, 1869-1888. [CrossRef]

57. Burini, D.; Chouhad, N. Hilbert method toward a multiscale analysis from kinetic to macroscopic models for active particles. Math. Model. Methods Appl. Sci. 2017, 27, 1327-1353. [CrossRef]

58. Burini, D.; Chouhad, N. A multiscale view of nonlinear diffusion in biology: From cells to tissues. Math. Model. Methods Appl. Sci. 2019, 29, 791-823. [CrossRef]

59. Poyato, D.; Soler, J. Euler-type equations and commutators in singular and hyperbolic limits of kinetic Cucker-Smale models. Math. Model. Methods Appl. Sci. 2017, 27, 1089-1152. [CrossRef]

60. Hoogendoorn, L.; Bovy, P.; Daamen, W. Walking infrastructure design assignment by continuous space dynamic assignment modeling. J. Adv. Transp. 2004, 38, 69-92. [CrossRef]

61. Venuti, F.; Bruno, L. Crowd structure interaction in lively footbridges under synchronous lateral excitation: A literature review. Phys. Life Rev. 2009, 6, 176-206. [CrossRef]

(c) 2019 by the authors. Licensee MDPI, Basel, Switzerland. This article is an open access article distributed under the terms and conditions of the Creative Commons Attribution (CC BY) license (http:/ / creativecommons.org/licenses/by/4.0/). 

Article

\title{
Particle Methods Simulations by Kinetic Theory Models of Human Crowds Accounting for Stress Conditions
}

\author{
Ahmed Elaiw ${ }^{1, *,+}$ and Yusuf Al-Turki ${ }^{2,+}$ \\ 1 Department of Mathematics, Faculty of Science, King Abdulaziz University, P.O. Box 80203, \\ Jeddah 21589, Saudi Arabia \\ 2 Department of Electrical and Computer Engineering, King Abdulaziz University, \\ Jeddah 21589, Saudi Arabia; yaturki@yahoo.com \\ * Correspondence: a_m_elaiw@yahoo.com; Tel.: +966-567573631 \\ + These authors contributed equally to this work.
}

Received: 19 October 2019; Accepted: 7 December 2019; Published: 19 December 2019

check for updates

\begin{abstract}
This paper tackles the problem of simulating the dynamics of human crowds in high density conditions on venues which include internal obstacles and in the interaction between two crowd streams moving in two opposite directions. The role of stress condition is taken into account as simulations aim at providing a support to crisis managers in charge of reducing the risk of incidents. The rationale of the modeling approach is that kinetic theory approach, where individual interactions, which might be nonlinearly additive, non symmetric, and non nonlocal, lead to collective behaviors to be examined towards safety problems.
\end{abstract}

Keywords: Crowd dynamics; kinetic models; stress conditions; boundary conditions; safety

\section{Plan and Aims of the Paper}

This paper tackles the problem of the simulation of crowd dynamics for the case studies briefly described in the abstract. The contents of our paper follows the critical analysis developed in [1] which has suggested to select the kinetic theory approach as the most appropriate to simulate crowd dynamics pedestrians in high density conditions taking into account both the level of stress in the crowd and the specific geometry of the areas, where the crowd moves. An additional feature, which is worth to be considered, is the physical quality of the venue.

The contents of our paper is mainly focused on simulations developed with the aim of enlightening the onset of conditions which may threaten the safety of pedestrians. Motivations to develop this study can be found in the paper [2] which starts by recalling the incident of 24 September 2015, on the Jamarat bridge in Makkah, and subsequently reports about specific features of human crowds to be carefully considered in the modeling. Most of the reasonings in [2] support the aforementioned selection of the mesoscopic scale to represent and model the class of systems under consideration as kinetic theory tools allow to account for the heterogenous behavior within the crowds.

Our paper refers to achievements known in the literature, recently reviewed in [3], to select the model and focuses on the development of simulations to enlighten the following features: the role of obstacles in the generation of overcrowded areas and the dynamics of streams moving towards opposite directions. Both types of simulations are critically analyzed within the general framework of crisis situations. Focusing on empirical data and model validation the following research contributions should be indicated $[4,5]$ referring to the specific features of the velocity and flow diagrams.

Section 2 presents the main achievement of this paper, namely simulations related to two specific case studies. The first one focuses on the dynamics in a venue constituted by parallel walls and 
which includes internal obstacles in the central part, while the second one focuses on a flow of two streams of crowds moving towards opposite directions in a corridor. The main objective of this type of simulations consists in showing how the dynamics of fragmentation and high density develops depending on the overall density and level of stress and how stress can generate overcrowding areas thus leading to potentially dangerous situations.

The mathematical model selected for the simulations includes, as we shall see, two key parameters, namely a first parameter models the physical quality of the area from a minimal value corresponding to the worst quality conditions which prevent the motion to the highest value which promotes fast motion, where the quality of the venue is induced by lighting, slopes, narrow corridors, stairs etcetera, while a second parameter accounts for the aforementioned level of stress which is also related to local density conditions.

Section 3 shows how the simulations presented in the previous section can be used to support crisis managers. A critical analysis addresses the computational requirements and discusses the possible use of machine learning techniques to enhance the simulation of human crowds. Some research perspectives are proposed in this section.

It is worth stressing that a correct level of the parameter modeling stress, which means a rational choice between the search of the attraction to the stream and the natural search to avoid overcrowded areas, is 0.4 . Then, increasing the value from 0.4 to 0.8 increases the attraction to the stream which is a critical situation, while higher values correspond to panic, namely to extreme danger.

\section{Selection of a Crowd Model and Simulations}

This section includes, as mentioned, the main results of our paper. The presentation is focused on the following four topics: Key requirements of the computational approach, selection of a mathematical model, simulations of a crowd flow on a bridge with internal obstacles, and a simulation of counter-flows. Each of them is treated in the next subsections. These achievements open to a critical analysis proposed in the next section which looks ahead to safety problems.

\subsection{Requirements of the Computational Approach}

The design of computational codes is related to the scale which has been selected for the modeling approach. In fact, different numerical tools are used for each scale, namely, time integration methods, such as Runge-Kutta methods, for large systems of ordinary differential equations, particle methods in the case of the kinetic theory approach, and finite differences or volumes in the case of the macroscopic approach.

In general, the requirements demanded to simulations essentially are the accuracy in computing local density and velocity and computational times lower or equal to the real time of the crowd. The former is a general requirement to for all types of applications, while the latter applies mainly on crisis management. Therefore, simulations are required to describe the flow patterns focusing both on local density and velocity under the constraint to keep the computational time below a critical time related to the specific dynamics which is investigated. Let us define precisely these two features.

From distribution function to macroscopic variables: The dependent variable of the kinetic theory approach is the probability distribution function over the microscopic state of walkers. As it is known,

Flow patterns: The key derived dependent variable is the density as it needs to be kept below a critical value, for safety reasons. Since dimensionless quantities will be used, the safety level is given by a "reasonable" number of walkers per square meter referred to the maximal "occupancy" number given by 7 walkers in the square meter. Assuming that a safe number is 4 , then the safety threshold is 4/7. In fact, when the dimensionless local density approaches to one, walkers can injury themselves to gain space. Further, increasing density increases the parameter-introduced later-which, in turn, promotes the increasing of density.

Critical time: Each dynamics has a critical time to achieve a certain objective, for instance the exit time increases with the density within the area. An additional effect is that when the density is low, namely the crowd is rarefied, the exit time decreases with the level of stress as the speed increases 
with this parameter. On the other hand, when the density is high, namely the crowd is dense, the exit time increases with the said $\times$ level of stress as even if speed increases with this parameter, walker unconsciously move towards dense areas and subsequently are obliged to decrease the speed. This feature of the dynamics should be put in connection with density patterns. For instance, large times are not acceptable in connection with high densities because of the probability of injuries increases.

\subsection{Selection of a Computational Model}

Methods of the kinetic theory approach to model human crowds, as reviewed in [1], have been initiated in [6] for crowds in unbounded domains and further developed in [7] to account for the interactions with walls and obstacles as well as for different levels of stress, while propagation of stress by contagion has been studied in [8], [9] and [10]. Derivation of models at the macroscopic scale from the microscopic scale can be obtained by development of Hilbert type approaches [11-14]. The pertinent bibliography on crowd modeling has been already reported in [1] focusing specifically on human crowds. Therefore only the essential references are reported in this present paper. The reader interested to a survey, where crowd dynamics, is related to the modeling of vehicles and swarms, is referred to the recent survey [3] which provides and exhaustive report of the bibliography in the field, where all technical details, which are not repeated here, are available.

The modeling approach is such that walkers, which constitute the sub-system at the microscopic scale, are modeled as active particles. These have the ability of expressing their own heterogeneously distributed walking strategy, called activity, which depends both on the level of the stress and on the physical quality of the venue. Interaction of different groups is considered for a crowd of individuals who share common features and walking strategy. The subdivision of the crowd into groups permits one to model different walking abilities and strategies.

We suppose that the motion is on a plane and that polar coordinates are used for the velocity of walkers, namely the velocity is described by the speed $v$ and by the velocity direction $\varphi$. Dimensionless quantities are used for the speed which is divided by a limit velocity expressed by a fast walker in a high quality venue whose geometry is denoted by $\Sigma$. Accordingly, the collective state of each group is described by a distribution function over the microscopic state of walkers:

$$
f_{i}=f_{i}(t, \mathbf{x}, v, \varphi ; \alpha, \beta), \quad \mathbf{x} \in \Sigma, \quad v \in[0,1], \quad \varphi \in[0,2 \pi),
$$

where the subscript $i=1, \ldots, n$ labels each group of walkers.

Generally, it is convenient referring $f_{i}$ to the maximum packing density. In practice about seven walkers per square meter. In addition, two dimensionless parameters can be introduced: $\alpha \in[0,1]$ and $\beta \in[0,1]$. Their physical meaning is as follows:

- $\alpha$ models the quality of the venue, where $\alpha=0$ corresponds to worst quality which, in practice, prevents motion, while $\alpha=1$ models high quality which can allows high speeds, however depending on the local density, as increasing density reduces locally the speed.

- $\quad \beta$ models the level of stress, where $\beta=0$ corresponds to absence of concentration, $\beta=0.5$ to a normal level of stress which is needed by a proper self-organization of the motion, and $\beta=1$ models the highest level of stress. The connection between level of stress and local density can be modeled by simple functions which depict how $\beta$ is not a fixed parameter, but it grows with the local density when the said density pass a critical threshold, e.g., $\rho_{c} \geq 0.5$.

Velocity weighted moments of $f_{i}$ lead, under integrability conditions, to compute macroscopic observable quantities. For instance, the total density is defined as follows:

$$
\rho(t, \mathbf{x})=\sum_{i=1}^{n} \rho_{i}(t, \mathbf{x})=\sum_{i=1}^{n} \int_{0}^{1} \int_{0}^{2 \pi} f_{i}(t, \mathbf{x}, v, \varphi ; \alpha, \beta) v d v d \varphi,
$$

where $\rho_{i}$ is the local density of each functional subsystem, while the flow is computed by 


$$
\begin{aligned}
\mathbf{q}(t, \mathbf{x}) & =\sum_{i=1}^{n} \mathbf{q}_{i}(t, \mathbf{x}) \\
& =\sum_{i=1}^{n} \int_{0}^{1} \int_{0}^{2 \pi}(v \cos \varphi \mathbf{i}+v \sin \varphi \mathbf{j}) f_{i}(t, \mathbf{x}, v, \varphi ; \alpha, \beta) v d v d \varphi,
\end{aligned}
$$

where $\mathbf{i}$ and $\mathbf{j}$ are the unit vectors of a plane cartesian frame. Higher order moments provide additional information.

Mathematical models consist in a differential system suitable to describe the dynamics in time and space of the aforementioned probability distribution functions. Kinetic models are obtained referring to the elementary volume in the space of the microscopic states and by equating the variation of particles (walkers) due to transport in the said volume to the inlet-outlet fluxes induced by interactions. As it is known, the key problem consists in the modeling of the interactions which generate the said fluxes.

A possible derivation rationale has been developed in [7]. Our paper reports, without repeating equations, available in [3,7], the key assumptions that lead to the model recalling that both speed and density have been referred to the highest admissible values so that these quantities take value in $[0,1]$. Specifically we look for a minimal model suitable, however, to capture the main features of human crowds.

1. Interaction rate: A simple model of the rate of interaction between walkers is obtained by assuming that this rate is constant.

2. Walking strategy: Walkers first select the walking direction, which ends up to correspond to the velocity direction, and subsequently adapt the speed to the local density pattern in their visibility zone.

3. Selection of the velocity direction: According to [7], the strategy which leads to the velocity direction $\varphi$ is a weighted selection out of three directions: (i) towards the trajectory going to the exit, (ii) attraction by the low gradient path, (iii) attraction by the main stream. The selection is driven by the local density $\rho$ and the parameter $\beta$ which models the level of stress. In more detail, attraction (ii) is enhanced by $\rho$, while attraction (iii) is enhanced by $\beta$.

4. Perception of the density along the velocity direction: Once a velocity direction has been selected walkers perceive a density different from the real one as follows:

$$
\begin{aligned}
& \partial_{\varphi}[\rho] \geq 0 \quad \Rightarrow \quad \rho_{\varphi}[\rho]=\rho+\frac{\partial_{\varphi}[\rho]}{\sqrt{1+\left(\partial_{\varphi}[\rho]\right)^{2}}}(1-\rho), \\
& \partial_{\varphi}[\rho]<0 \quad \Rightarrow \quad \rho_{\varphi}[\rho]=\rho+\frac{\partial_{\varphi}[\rho]}{\sqrt{1+\left(\partial_{\varphi}[\rho]\right)^{2}}} \rho,
\end{aligned}
$$

where $\partial_{\varphi}$ denotes the derivative along the direction $\varphi$. Hence, $\rho_{\varphi}[\rho]$ is higher (lower) that the real one in the presence of positive (negative) gradients, by a model which accounts for lower and higher bounds:

$$
\partial_{\varphi}[\rho] \rightarrow \infty \Rightarrow \rho_{\varphi} \rightarrow 1, \quad \partial_{\varphi}[\rho]=0 \Rightarrow \rho_{\varphi}=\rho, \quad \partial_{\varphi}[\rho] \rightarrow-\infty \Rightarrow \rho_{\varphi} \rightarrow 0 .
$$

5. Adjustment of the speed: Walkers, after perception of the density, adjust their speed to the perceived density conditions. The new speed $v_{\varphi}$ can be modeled as follows:

$$
\begin{aligned}
& \partial_{\varphi}[\rho] \geq 0 \quad \Rightarrow \quad v_{\varphi}[\rho]=v-\alpha \rho_{\varphi}[\rho] v, \\
& \partial_{\varphi}[\rho]<0 \quad \Rightarrow \quad v_{\varphi}[\rho]=v+\alpha \rho_{\varphi}[\rho](1-v),
\end{aligned}
$$

6. Stress-density reciprocal influence: A key feature of the dynamics is the interaction between local density and level of stress as when the local density tends to the maximal value also the local stress tends to limit value $\alpha=1$. 
7. Boundary conditions: The statement of the mathematical problem requires the implementation of boundary conditions which depend on the distance from the wall measured along the direction of motion. When this distance tends to zero the attraction by the trajectory towards the exit is enhanced with respect to the other directions.

The integro-differential nature of kinetic equations, along with the decision process described above by which pedestrians select their preferred velocity direction and speed, leads to a computational model whose mathematical structure is quite involved. Monte Carlo particle methods are ideally suited for solving such an equation while keeping the computational burden at a reasonable level. The basic idea consists in representing the distribution function by a number of computational particles. These particles move in the computational domain and interact according to stochastic rules derived from the governing kinetic equation. The macroscopic fields are obtained through weighted averages of the particle properties.

Compared to deterministic methods of solution, Monte Carlo simulations shows some important advantages such as computational efficiency and the capability of taking into account sophisticated individual decision processes. This statement refers to kinetic type models only, where the implementation of deterministic methods involves well known technical difficulties.

\subsection{Simulations of Crowds over a Bridge with Internal Obstacles}

This subsection shows how the mathematical and computational tools presented in Section 2.2, can be applied to reproduce the flow of human crowds venues which show geometrical features analogous to that of the Jamarat bridge. Special focus is on the role of columns placed in the middle of the way as these columns might become a source of incidents. As we shall see, useful insights can be obtained which can provide suggestions on how the geometric design of the venue might be revised for optimizing the pedestrian flow and how crisis managers can use the output of simulations to improve safety conditions. It is worth stressing that our paper does not aim at a simulation specifically focused on the aforementioned venue, but at developing mathematical tools which can be specifically addressed to such objective which would require specific data and commitments.

The pioneer approach to safety problems belongs to Helbing and coworkers [15-17], while several developments have been recently developed as reported in the bibliography of [8]. A recent research work [18] shows how a systems approach to safety problems can be developed, where a key feature consists in accounting for the connection between local density and stress. Indeed, this is one of the key feature of our paper.

The development of the computational approach first accounts for the requirements presented in the previous section, namely dealing with a crowd with large size, high performance computing suitable to keep the computational time lower that the real simulated time, and accounting for the level of stress in the crowd. Some interesting results have been proposed in the literature concerning simulations of kinetic type models $[19,20]$. Our computational experiments have suggested the use of Monte Carlo particle methods introduced by Bird [21], within the general framework of computational methods for Boltzmann type equations [22], and subsequently extended by various authors by modifying the method depending on the specific system under consideration, for instance [23,24], see also Section 6 of [3] which is specifically focused on crowd and swarm dynamics, while the survey [25] exhaustively covers this challenging field of scientific computing.

It is worth enlightening some conceptual differences with respect to the recently published paper [20], where some interesting simulations have been developed concerning evacuation dynamics and counter-flows. These differences allow to make more precise the contribution of our paper.

- The number of pedestrians in the simulations is of a higher order, namely of the order $\mathrm{f} 10^{3}$ instead of $10^{2}$.

- The velocity is continuous rather than discrete in a fixed number of directions, while the speed is not related to the velocity diagram, but it accounts for local gradients. 
- The individual decision processes which leads to the selection of the velocity direction account for the presence of obstacles.

- The dynamics accounts for the different level of stress and of their relation to local density.

- A stochastic computational method has been developed to take into account all specific features indicated in the above items.

However, an extensive simulation campaign is, however, beyond the scope of this paper and only sample simulation results are briefly discussed in the following with the aim of validating the computational code.

Figures 1 and 2 show the behavior of a crowd composed of 1000 people and 2000 people for different times, respectively. Pedestrians are initially uniformly distributed in the entrance ramp with a level of stressful conditions of $\beta=0.6$. As the simulation starts, the crowd splits into two groups which follow the shortest path to avoid the obstacle. In the attempt to keep constant their walking speed, however, a few pedestrians move away from the more congested areas and head for the empty region between the two groups.

It is apparent that in the higher density case potentially dangerous conditions occur near the corners where the sudden change in geometry leads to very high congestion levels. Additional simulations have shown that increasing the number of individuals in the crowd enhances the onset of high density zones. Analogous effect appears for increasing values of the parameter modeling the level of stress, simulations are not reported to avoid redundancy of similar simulations.

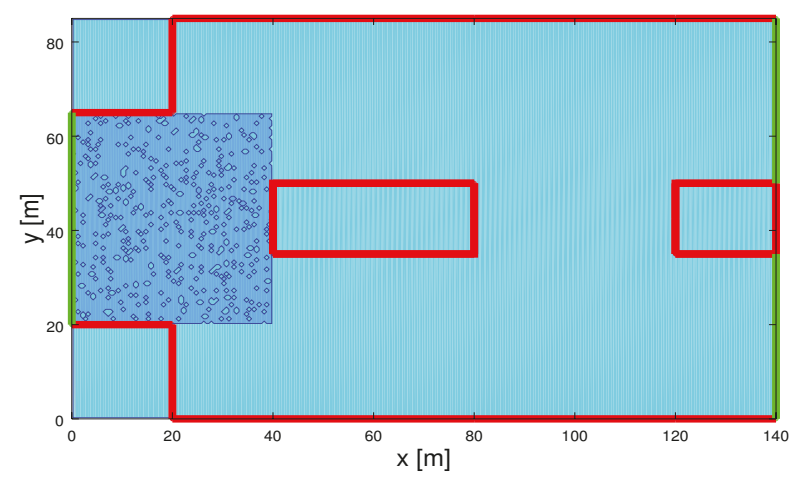

(a) $t=0 \mathrm{~s}$.

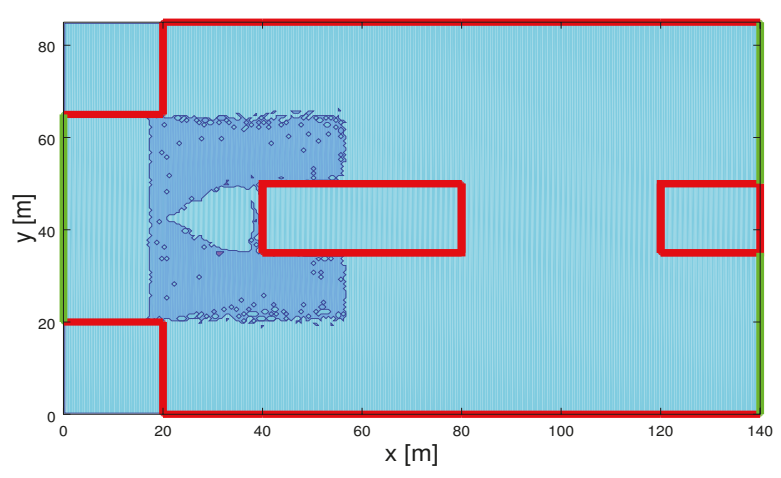

(b) $t=20 \mathrm{~s}$.

Figure 1. Cont. 


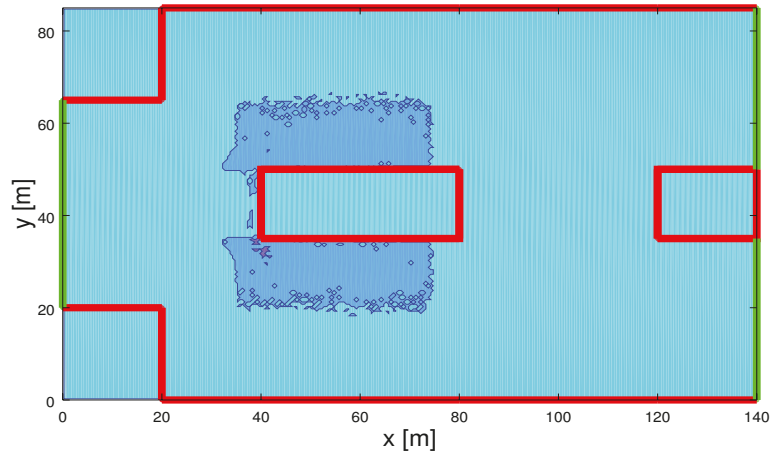

(b) $t=40 \mathrm{~s}$.

Figure 1. Density contour plot of crowds composed of 1000 pedestrians for different times.

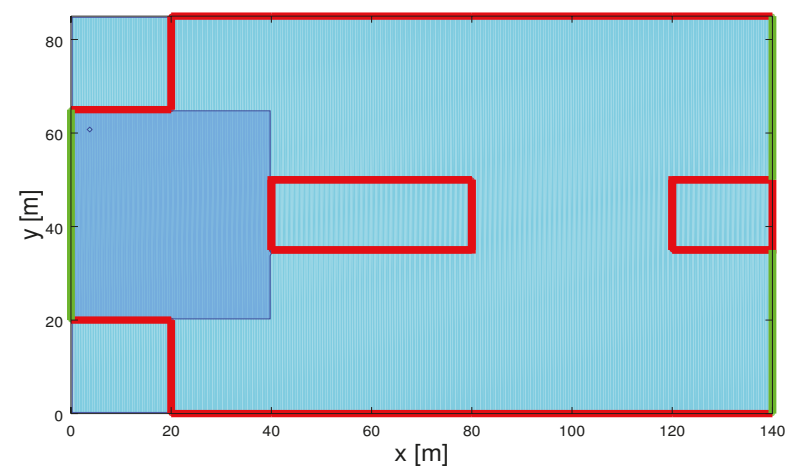

(a) $t=0 \mathrm{~s}$.

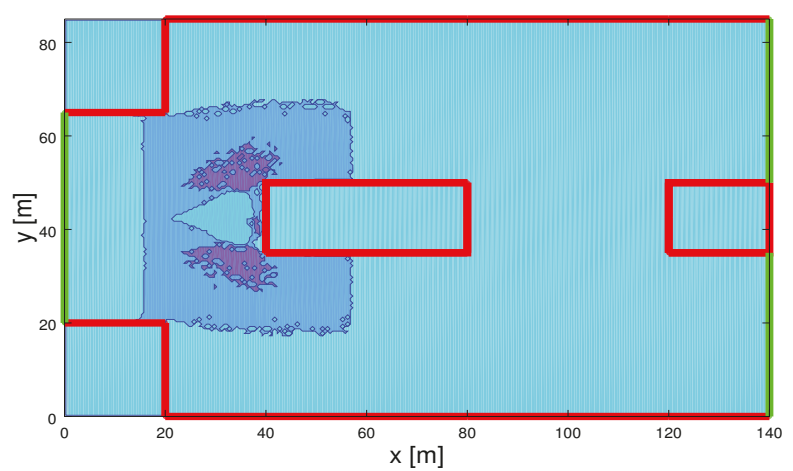

(b) $t=20 \mathrm{~s}$.

Figure 2. Cont. 


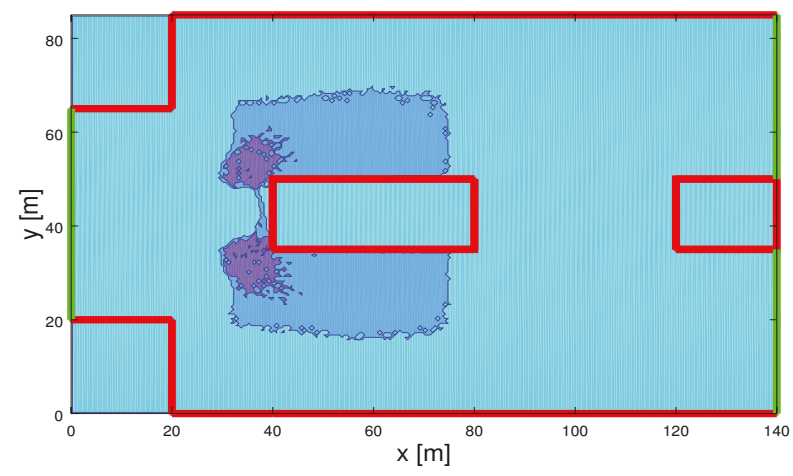

(b) $t=40 \mathrm{~s}$

Figure 2. Density contour plot of crowds composed of 2000 pedestrians for different times.

\subsection{Flows of Crowds Moving to Opposite Directions}

A different type of simulation is considered in this subsection once again having in mind safety problems. Namely we consider a crowd moving along a corridor being contrasted by a another crowd suddenly moving against. The level of stress in the first group is moderate, say normal, while the level of stress in the second group if high. Therefore, the second group forces against the first group.

This physical situation can appear if the second group has met a dangerous situation, for instance caused by an incident, and consequently a group has decided to invert the direction of motion to escape danger. The main interest of the simulations consists in showing if the dynamics exhibits fragmentation and subsequent localization of high levels of density-stress.

The flow patterns related to this type of dynamics are shown in Figures 3 and 4 which show how the flow pattern at $t=0$ is modified by the interactions between the two streams as well as by the interactions within walkers moving along the same direction.

Simulations show in Figure 3 the progressive formation of high concentration of density-stress patterns, while Figure 4 shows the persistence of high level of concentration even when the two groups have completely gone across each other. In more detail on the density patterns, both groups have an initial density of 3 persons per square meter, that can be considered a high density, but not yet critical. On the other hand, the interaction between these two groups generates concentration approaching 7 persons per square meter, namely a highly critical density as far as safety is concerned.

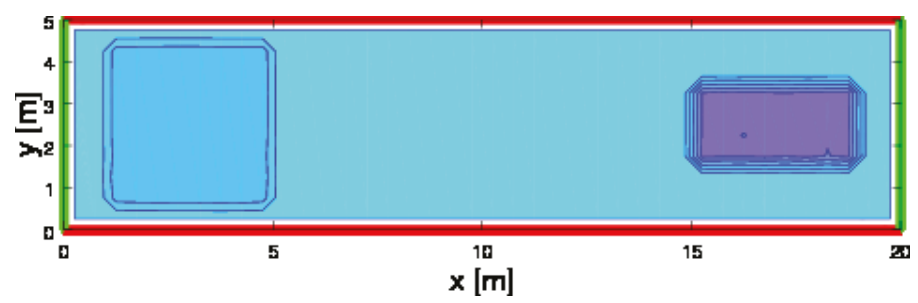

(a) $t=0 \mathrm{~s}$.

Figure 3. Cont. 


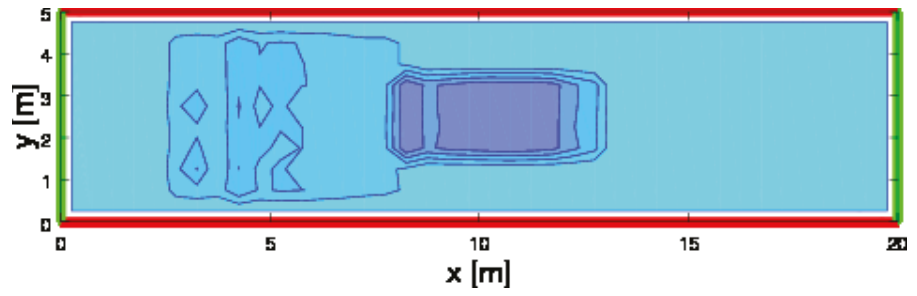

(b) $t=6 \mathrm{~s}$.

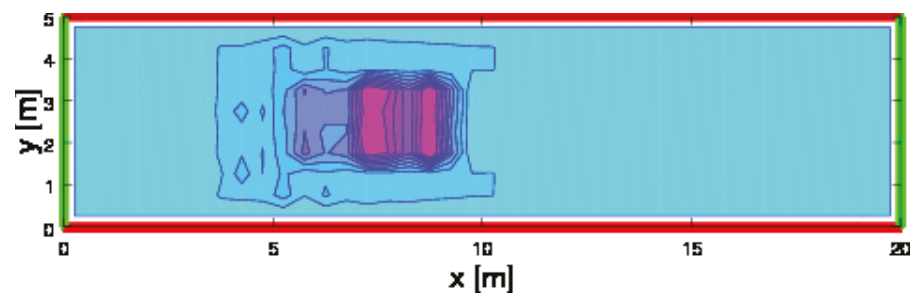

(c) $t=10 \mathrm{~s}$.

Figure 3. Flow patterns, at different times, in the interactions of two streams moving towards opposite directions.

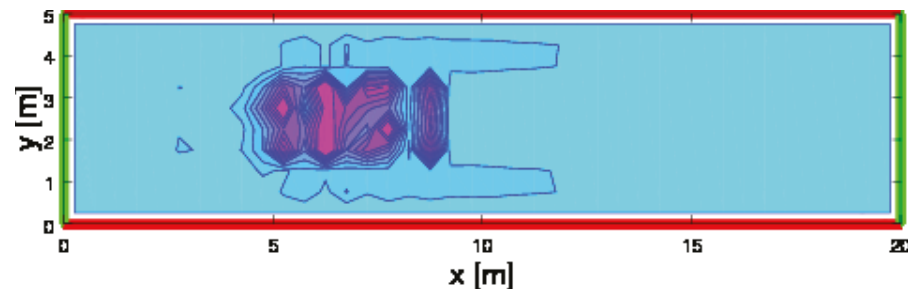

(a) $t=14 \mathrm{~s}$.

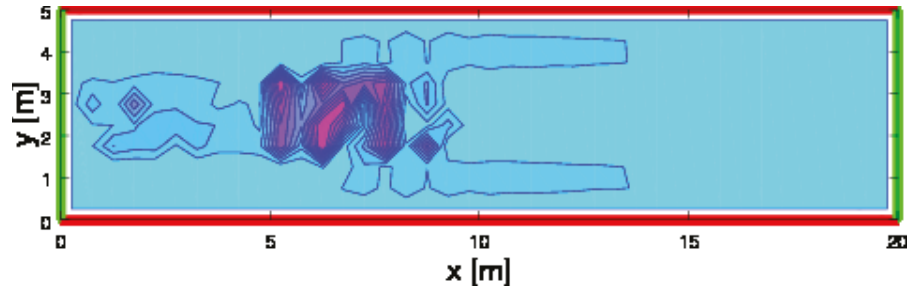

(b) $t=18 \mathrm{~s}$.

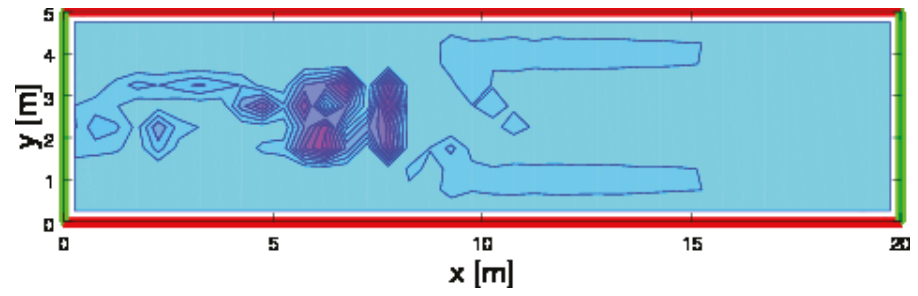

(c) $t=22 \mathrm{~s}$.

Figure 4. Flow patterns, for large times, in the interactions of two streams moving towards opposite directions. 
The sample simulations presented above provide a detailed description of the local density and velocity pattern in each point of the area where the crowd moves by means of the visualization of high concentration zones. In addition, simulations also show how the localization of high concentration zones evolves in time and space.

\section{Critical Analysis towards Safety Problems}

As we have seen, advanced mathematical and computational tools have been used. However, we do not naively think that these tools can be simplified, as their use can be planned and optimized within a proper research plan. However, some perspective ideas are proposed in the following items which focus both on safety problems and research perspectives.

Concerning safety problems the following perspective is proposed: A huge number of simulations corresponding the all possible variety of situations can be developed by using the approach proposed in our paper. Then, these simulations can be stored in a database. The subsequent use of the database consists in training crisis managers by identification of a broad variety of the possible actions that can effectively reduce the crisis situation. In addition, the database can be used also to train leaders who in the crowd can contribute, by addressing the walking dynamics, to reduce the risk of safety problems. More in general, simulations can provide a detailed description of the role of the geometry and quality of the venue over the dynamics in space of the aforementioned density patterns.

This effort needs additional work with respect to the development of computational schemes. The systems approach [18], the engineering vision of safety problems [26,27], computer vision and virtual reality [28-30], while a deep study of the psychology of crowds [31] can contribute to the assessment of all specific features to be taken into account in the modeling approach and hence in the machine learning design.

Concerning research perspectives we bring the attention of the reader to multiscale problems: A multiscale vision of crowd modeling can be important looking at different aspects of the development of computational modeling and of the validation of models. Two key issues have to be taken into account in the development of a multiscale modeling, namely the derivation of models at all scales accounting for the same principles and derivation of models at the higher scale from the underlying description at the lower scale. In principles these two different views can join into a unified multiscale vision. Some work in this direction has been has been developed for crowds in unbounded domains [32].

This perspective appears to be a key requirement in the search of a valid modeling approach. The contributions available in the literature still need additional work which cannot skip over the need of sophisticated analytic tools to achieve this result. A deep analysis of the complex behaviors at the microscopic scale [33-35] which can include non symmetrical behaviors [36], can contribute to model the link between individual and collective behaviors.

Development of these two specific perspectives would definitely contribute to design models of crowd dynamics effectively useful to applications.

Author Contributions: Conceptualization, A.E. and Y.A.-T.; methodology, A.E.; formal analysis, Y.A.-T.; investigation, A.E.; writing original draft preparation, Y.A.-T.; writing review and editing, A.E. and Y.A.-T.; project administration, A.E. All authors have read and agreed to the published version of the manuscript.

Funding: This project was funded by the Deanship of Scientific Research (DSR), King Abdulaziz University, Jeddah, under grant No. (DF-013-130-1441). The authors, therefore, gratefully acknowledge DSR technical and financial suppor.

Conflicts of Interest: The authors declare no conflict of interest.

\section{References}

1. Elaiw, A.; Al-Turki, Y.; Alghamdi, M. A critical analysis of behavioural crowd dynamics: From a modelling strategy to kinetic theory methods. Symmetry 2019, 11, 851. [CrossRef]

2. Moussaïd, M. Moins la foule est dense, plus elle est imprévisible, 2019. Available online: https://www. pourlascience.fr/ (accessed on 18 October 2019). 
3. Albi, G.; Bellomo, N.; Fermo, L.; Ha, S.-Y.; Kim, J.; Pareschi, L.; Poyato, D.; Soler, J. Traffic, crowds, and swarms. From kinetic theory and multiscale methods to applications and research perspectives. Math. Models Methods Appl. Sci. 2019, 29, 1901-2005. [CrossRef]

4. Schadschneider, A.; Chraibi, M.; Seyfried, A.; Tordeux, A.; Zhang, J. Pedestrian dynamics: From empirical results to modeling. In Crowd Dynamics Voume 1-Theory Models and Safety Problems; Gibelli, L., Bellomo, N., Eds.; Springer: Berlin/Heidelberg, Germany, 2018; pp. 63-102.

5. Schadschneider, A.; Klingsch, W.; Kläpfel, H.; Kretz, T.; Rogsch, C.; Seyfried, A. Evacuation dynamics: Empirical results, modeling and applications. In Encyclopedia of Complexity and System Science; Springer: Berlin/Heidelberg, Germany, 2009; pp. 3142-3176.

6. Bellomo, N.; Bellouquid, A.; Knopoff, D. From the micro-scale to collective crowd dynamics. Multiscale Model. Simul. 2013, 11, 943-963. [CrossRef]

7. Bellomo, N.; Gibelli, L. Toward a mathematical theory of behavioral-social dynamics for pedestrian crowds. Math. Models Methods Appl. Sci. 2015, 25, 2417-2437. [CrossRef]

8. Bellomo, N.; Gibelli, L.; Outada, N. On the interplay between behavioral dynamics and social interactions in human crowds. Kinet. Relat. Models 2019, 12, 397-409. [CrossRef]

9. Bertozzi, A.L.; Rosado, J.; Short, M.B.; Wang, L. Contagion shocks in one dimension. J. Stat. Phys. 2015, 158, 647-664. [CrossRef]

10. Wang, L.; Short, M.; Bertozzi, A.L. Efficient numerical methods for multiscale crowd dynamics with emotional contagion. Math. Models Methods Appl. Sci. 2017, 27, 205-230. [CrossRef]

11. Bellomo, N.; Bellouquid, A.; Chouhad, N. From a multiscale derivation of nonlinear cross-diffusion models to Keller-Segel models in a Navier-Stokes fluid. Math. Models Methods Appl. Sci. 2016, 26, 2041-2069. [CrossRef]

12. Burini, D.; Chouhad, N. Hilbert method toward a multiscale analysis from kinetic to macroscopic models for active particles. Math. Models Methods Appl. Sci. 2017, 27, 1327-1353. [CrossRef]

13. Burini, D.; Chouhad, N. A multiscale view of nonlinear diffusion in biology: From cells to tissues. Math. Models Methods Appl. Sci. 2019, 29, 791-823. [CrossRef]

14. Burini, D.; De Lillo, S. On the complex interaction between collective learning and social dynamics. Symmetry 2019, 11, 967. [CrossRef]

15. Helbing, D. Traffic and related self-driven many-particle systems. Rev. Mod. Phys. 2001, 73, 1067-1141. [CrossRef]

16. Helbing, D.; Farkas, I.; Vicsek, T. Simulating dynamical feature of escape panic. Nature 2000, 407, 487-490. [CrossRef] [PubMed]

17. Helbing, D.; Johansson, A. Pedestrian crowd and evacuation dynamics. In Encyclopedia of Complexity and System Science; Springer: Berlin/Heidelberg, Germany, 2009; pp. 6476-6495.

18. Yin, J.; Zheng, X.-M.; Tsaur, R.C. Occurrence mechanism and copying paths of accidents of highly aggregated tourist crowds based on systems dynamics. PLoS ONE 2019, 14. [CrossRef] [PubMed]

19. Elmoussaoui, A.; Argoul, P.; Elrhabi, M.; Hakim, A. Discrete kinetic theory for 2-Dmodeling of a moving crowd: Application to the evacuation of a non-connected bounded domain. Comput. Math. Appl. 2018, 75, 1159-1180. [CrossRef]

20. Kim, D.; Quaini, A. A kinetic theory approach to model pedestrian dynamics in bounded domains with obstacles. Kinet. Relat. Models 2019, 12, 1273-1296. [CrossRef]

21. Bird, G.A. Molecular Gas Dynamics and the Direct Simulation of Gas Flows; Oxford University Press: Oxford, UK, 1994.

22. Aristov, V.V. Direct Methods for Solving the Boltzmann Equation and Study of Nonequilibrium Flows; Springer: New York, NY, USA, 2001.

23. Barbante, P.; Frezzotti, A.; Gibelli, L. A kinetic theory description of liquid menisci at the microscale. Kinet. Relat. Models 2015, 8, 235-254.

24. Pareschi, L.; Toscani, G. Interacting Multiagent Systems: Kinetic Equations and Monte Carlo Methods; Oxford University Press: Oxford, UK, 2013.

25. Dimarco, G.; Pareschi, L. Numerical methods for kinetic equations. Acta Numer. 2014, 23, 369-520. [CrossRef]

26. Ronchi, F.; Nieto Uriz, F.; Criel, X.; Reilly, P. Modelling large-scale evacuation of music festival. Fire Saf. 2016, 5, 11-19. [CrossRef] 
27. Ronchi, E.; Nilsson, D. Pedestrian movement in smoke, Data and Modeling approaches. In Crowd Dynamics Voume 1-Theory Models and Safety Problems; Gibelli, L., Bellomo, N., Eds.; Springer: Berlin/Heidelberg, Germany, 2018; pp. 37-62.

28. Kinateder, M.; Wirth, T.D.; Warren, W.H. Crowd dynamics in virtual reality. In Crowd Dynamics Voume 1Theory Models and Safety Problems; Gibelli, L., Bellomo, N., Eds.; Springer: Berlin/Heidelberg, Germany, 2018; pp. 11-12.

29. Talmann, D.; Musse, S.R. Crowd Simulation; Springer: Berlin/Heidelberg, Germany, 2007.

30. Zhan, B.; Monekosso, D.-N.; Remagnino, P.; Velastin, S.A.; Xu, L.-Q. Crowd analysis: A survey. Mach. Vis. Appl. 2008, 19, 345-357. [CrossRef]

31. Wijermans, N.; Conrado, C.; van Steen, M.; Martella, C.; Li, J.-L. A landscape of crowd management support: An integrative approach. Saf. Sci. 2016, 86, 142-164. [CrossRef]

32. Bellomo, N.; Bellouquid, A. On multiscale models of pedestrian crowds from mesoscopic to macroscopic. Commun. Math. Sci. 2015, 13, 1649-1664. [CrossRef]

33. Bellomo, N.; Knopoff, D.; Soler, J. On the difficult interplay between life "complexity" and mathematical sciences. Math. Models Methods Appl. Sci. 2013, 23, 1861-1913. [CrossRef]

34. Knopoff, D.; Nieto, J.; Urrutia, L. Numerical simulation of a multiscale cell motility model based on the kinetic theory of active particles. Symmetry 2019, 11, 1003. [CrossRef]

35. Moussaid, M.; Helbing, D.; Garnier, S.; Johanson, A.; Combe, M.; Theraulaz, G. Experimental study of the behavioral underlying mechanism underlying self-organization in human crowd. Proc. R. Soc. B Biol. Sci. 2009, 276, 2755-2762. [CrossRef] [PubMed]

36. Burger, M.; Düring, B.; Kreusser, L.M.; Markowich, P.A.; Schönlieb, C.-B. Pattern formation of a nonlocal, anisotropic interaction model. Math. Models Methods Appl. Sci. 2018, 28, 409-451. [CrossRef]

(c) 2019 by the authors. Licensee MDPI, Basel, Switzerland. This article is an open access article distributed under the terms and conditions of the Creative Commons Attribution (CC BY) license (http:/ / creativecommons.org/licenses/by/4.0/). 
MDPI

St. Alban-Anlage 66

4052 Basel

Switzerland

Tel. +41616837734

Fax +41 613028918

www.mdpi.com

Symmetry Editorial Office

E-mail: symmetry@mdpi.com www.mdpi.com/journal/symmetry

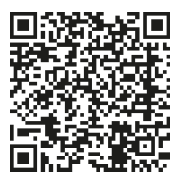



MDPI

St. Alban-Anlage 66

4052 Basel

Switzerland

Tel: +41 616837734

Fax: +41 613028918

www.mdpi.com 